\title{
Fluctuational electrodynamics for nonlinear materials in and out of equilibrium
}

\author{
DISSERTATION \\ for the award of the degree \\ "Doctor rerum naturalium" \\ of the Georg-August-Universität Göttingen \\ within the doctoral program \\ Physics \\ of the Georg-August University School of Science (GAUSS)
}

\author{
submitted by \\ Heino Soo \\ from Jõgeva, Estonia
}

Göttingen, 2019 


\section{Thesis committee}

Prof. Dr. Matthias Krüger,

Institut für Theoretische Physik,

Georg-August-Universität Göttingen

Prof. Dr. Reiner Kree,

Institut für Theoretische Physik,

Georg-August-Universität Göttingen

Prof. Dr. Steffen Schumann,

Institut für Theoretische Physik,

Georg-August-Universität Göttingen

\section{Members of the examination board}

Prof. Dr. Matthias Krüger,

Institut für Theoretische Physik,

Georg-August-Universität Göttingen

Prof. Dr. Reiner Kree,

Institut für Theoretische Physik,

Georg-August-Universität Göttingen

\section{Further members of the examination board}

Prof. Dr. Steffen Schumann,

Institut für Theoretische Physik,

Georg-August-Universität Göttingen

Prof. Dr. Marcus Müller,

Institut für Theoretische Physik,

Georg-August-Universität Göttingen

Prof. Dr. Peter Sollich,

Institut für Theoretische Physik,

Georg-August-Universität Göttingen

Prof. Dr. Fabian Heidrich-Meisner,

Institut für Theoretische Physik,

Georg-August-Universität Göttingen

\section{Date of the oral examination}

April 16, 2019 
To all the wonderful people in this world. 



\section{List of publications}

[85] Heino Soo and Matthias Krüger, Fluctuational electrodynamics for nonlinear media, EPL 115, 4 (2016), pp. 41002.

[84] Heino Soo, David S. Dean, and Matthias Krüger, Particles with nonlinear electric response: Suppressing van der Waals forces by an external field, Phys. Rev. E 95, 1 (2017), pp. 012151.

[86] Heino Soo and Matthias Krüger, Fluctuational electrodynamics for nonlinear materials in and out of thermal equilibrium, Phys. Rev. B 97, 4 (2018), pp. 045412. 



\section{Contents}

List of publications $\quad$ v

1. Introduction 1

1.1. Overview . . . . . . . . . . . . . . . . . . . 1

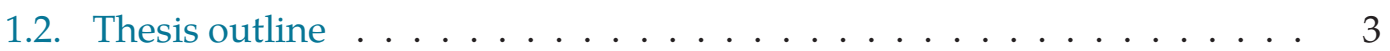

2. Combining electromagnetic fluctuations and nonlinear optics $\mathbf{5}$

2.1. From Maxwell's equations to linear fluctuational electrodynamics . . . . 5

2.1.1. The wave equation in Fourier space . . . . . . . . . . . . 5

2.1.2. Linear Helmholtz equation in operator notation . . . . . . . . 8

2.1.3. Fluctuations of the electromagnetic field . . . . . . . . . 10

2.2. Nonlinear optics . . . . . . . . . . . . . . . . . . . . . 15

2.2.1. The nonlinear polarizability . . . . . . . . . . . . . . . 15

2.2.2. Properties of the nonlinear susceptibilities . . . . . . . . . . 17

2.2.3. The nonlinear Helmholtz equation . . . . . . . . . . . . . . . 19

2.3. The general stochastic nonlinear Helmholtz equation . . . . . . . . . . . 21

2.3.1. Nonlinear optics with noise . . . . . . . . . . . . . . 21

2.3.2. The nonlinear response operator . . . . . . . . . . . . . 22

2.3.3. The effective nonlinear Helmholtz equation . . . . . . . . . . . 23

2.3.4. Fluctuations and noise . . . . . . . . . . . . . . 25

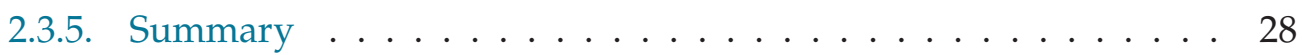

2.4. Perturbative treatment . . . . . . . . . . . . . . . . . . . . . . . 29

2.4.1. Approximating the nonlinear Helmholtz equation . . . . . . . . . 29

2.4.2. The effective nonlinear Helmholtz equation . . . . . . . . . . . . 31

2.4.3. The effective potential and dielectric function . . . . . . . . . . 32

2.4.4. The linear response . . . . . . . . . . . . . . . . . . . 33

2.5. Summary . . . . . . . . . . . . . . . . . . . . . 34

3. Fluctuational electrodynamics for nonlinear materials in equilibrium $\quad \mathbf{3 5}$

3.1. Electromagnetic fluctuations and noise in equilibrium . . . . . . . . . 36

3.1.1. Equilibrium fluctuations . . . . . . . . . . . . . . . 36

3.1.2. Equilibrium noise - equivalence of Rytov theory and the FDT . . 36 
3.2. The effective dielectric function in equilibrium . . . . . . . . . . . 38

3.2.1. $\tilde{\varepsilon}^{\text {eq }}$ for a planar surface - shape dependence . . . . . . . . . . . 40

3.2.2. $\tilde{\varepsilon}^{\text {eq }}$ for separated objects - combination formulas . . . . . . . . . . 41

3.2.3. $\tilde{\varepsilon}^{\text {eq }}$ for two parallel surfaces - distance dependence . . . . . . . . 48

3.2.4. Bulk, surface, and distance contributions for homogeneous objects 49

3.3. Example - equilibrium Casimir force . . . . . . . . . . . . 53

3.3.1. Modified power laws for frequency-independent materials . . . . 53

3.3.2. Force between an invisible plate and a perfect mirror . . . . . . . 57

3.4. Summary . . . . . . . . . . . . . . . . . . . . 57

4. Thermal imbalance in nonlinear systems and radiative heat transfer $\quad \mathbf{5 9}$

4.1. Fluctuations in the local thermal equilibrium approximation . . . . . . . 60

4.1.1. Non-equilibrium Rytov currents in the LTE approximation . . . . 60

4.1.2. Non-equilibrium fluctuations in the LTE approximation . . . . . 63

4.2. The effective dielectric function out of thermal equilibrium . . . . . . . . 64

4.2.1. The effective dielectric function in the LTE approximation . . . . 64

4.2.2. The non-equilibrium part to the effective dielectric function . . . 65

4.2.3. One object in vacuum - passive gain media . . . . . . . . . . . 66

4.3. Heat radiation and transfer in nonlinear systems . . . . . . . . . . . 69

4.3.1. A single arbitrary object - anomalous modes . . . . . . . . . . 69

4.3.2. Example a nonlinear nanosphere . . . . . . . . . . . . . 70

4.4. Summary . . . . . . . . . . . . . . . . . . . 72

5. Nonlinear fluctuations in the presence of external fields $\mathbf{7 3}$

5.1. Introductory example - van der Waals force in a constant field . . . . . 73

5.2. Stochastic scalar model - general approach . . . . . . . . . . . . 75

5.2.1. The phenomenological model . . . . . . . . . . . . . . 75

5.2.2. The fluctuations and effective noise . . . . . . . . . 76

5.2.3. The statistical properties of the effective noise . . . . . . . . 77

5.2.4. Zero-mean noise: the bare model . . . . . . . . . . . . . . . . 79

5.2.5. Summary and discussion about assumptions . . . . . . . . . . 82

5.3. Stochastic scalar model - perturbative approach . . . . . . . . . . . . 85

5.3.1. Perturbative models . . . . . . . . . . . . . . . . . 85

5.3.2. Perturbative noise and fluctuations . . . . . . . . . . . . . 87

5.3.3. Modelling non-equilibrium noise . . . . . . . . . . . . . . . 90

5.4. Nonlinear fluctuational electrodynamics with external fields . . . . . . 93

5.5. Summary . . . . . . . . . . . . . . . . . . . . . 95

6. Concluding remarks 9

6.1. Results and conclusions . . . . . . . . . . . . . . . . . . 97 
6.2. Open questions . . . . . . . . . . . . . . . . . . . . 98

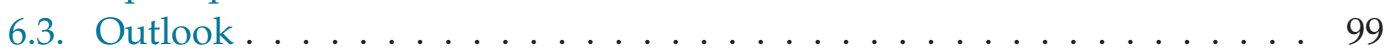

$\begin{array}{ll}\text { A. Functional derivatives } & 101\end{array}$

B. Green's functions for multilayer structures 103

B.1. Green's function for two parallel surfaces . . . . . . . . . . . . . . . . . . . . . . . . . . 103

B.2. $\operatorname{Im} \mathbb{G}(\mathbf{r}, \mathbf{r})$ in bulk . . . . . . . . . . . . . . . . . . . . . 104

B.3. $\operatorname{Im} \mathbb{G}(\mathbf{r}, \mathbf{r})$ for a single surface $\ldots \ldots \ldots \ldots \ldots \ldots$

$\begin{array}{lr}\text { C. Casimir force and the Lifshitz formula } & 107\end{array}$

C.1. General Casimir force . . . . . . . . . . . . . . . . . . . 107

C.2. Nonlinear Lifshitz formula . . . . . . . . . . . . . . . . . . . . 108

$\begin{array}{ll}\text { D. Heat transfer formula } & 111\end{array}$

$\begin{array}{lr}\text { Bibliography } & 113\end{array}$

$\begin{array}{lr}\text { Acknowledgments } & 121\end{array}$

$\begin{array}{lr}\text { Curriculum vitae } & 123\end{array}$ 



\section{Introduction}

\subsection{Overview}

The electromagnetic field is inherently stochastic, undergoing both thermal and quantum fluctuations. In many cases these fluctuations do not contribute to measurements simply because only the noise-average field is manipulated (for example in most optics experiments), and thus the deterministic, or averaged, Maxwell's equations [38] are often enough to describe the experiments. There are, however, many interesting phenomena which would not exist without fluctuations - the most prominent of them being fluctuational forces (temperature-dependent forces between neutral objects) and radiative heat transfer.

The theoretical description of these phenomena has historically evolved from very specific to more general systems. The fluctuational forces are often referred to as Casimir forces after the first description of the force between two semi-infinite neutral perfectly metallic plates, first given by Casimir in 1948 [19]. The theory was later generalized by Lifshitz for plates with arbitrary dielectric properties [59]. Of the same origin is also the force between two neutral particles, known as the Casimir-Polder (retarded limit) [20] or van der Waals force $[28,14]$. All these different forces share two similarities: they are of electromagnetic origin (they depend on the dielectric rather than chemical properties), and have an extremely short range as compared to the Coulomb force. Famously, this is used by spiders and geckos to attach to any surface - their feet are covered with extremely tiny hairs, which are able to get close enough to surfaces in order to activate this force. For two similar surfaces in contact, the average separation is normally limited by microscopic roughness.

The advancement of experimental methods for measuring forces [88, 56, 64, 15, 68] and heat transfer $[47,76,81,69,46]$, as well as the development of the microelectromechanical systems (MEMS) framework [34], has in turn lead to increased efforts in the past decade to develop theoretical models for these experiments. Of particular use is fluctuational electrodynamics (FE), a family of theories which relates the fluctuations of the electromagnetic field to the dielectric properties (for example reflection coefficients) in systems of arbitrary geometry. This can be accomplished with the help of the fluctuation-dissipation theorem (FDT) [1, 53, 40], Rytov currents [77, 58], 


\section{Introduction}

or macroscopic quantum electrodynamics $[62,63]$. The development of FE has lead to a wide range of theoretical results for arbitrary systems, such as Casimir forces in and out of equilibrium $[5,12]$, forces between moving objects [70], and radiative heat transfer $[77,71,29]$. More recent advances have moved toward basis-free trace formulas $[72,51,8,9,74,65]$, allowing for a convenient description of relevant experimental setups such as plate-sphere geometries.

While FE can be used, in principle, to calculate the forces and heat transfer between bodies of arbitrary shape and dielectric properties, most results require the materials to be purely linear. This assumption simplifies the calculations greatly, in large part due to the superposition principle: the electromagnetic field can be separated into an average and fluctuating part, which do not influence each other. However, doing this leaves out a vast field of physics, namely that of nonlinear optics (NLO), with many interesting effects such as frequency mixing, optical Kerr effect, and Raman/Brillouin scattering, to name a few $[80,13]$. The wider goal of this thesis is to incorporate the physics of NLO into FE.

Because of the breakdown of the superposition principle, including NLO into FE would possibly open an avenue into the interesting field of tunable Casimir forces $[73,23,4,78,97,22,90,92,17,25]$. While naturally occurring materials tend to exhibit rather weakly nonlinear behavior, strong lasers can overcome this. This is sub-optimal for our purposes, because of the low power of fluctuations at room temperature. The rise of nonlinear metamaterials $[42,57,10]$, however, gives a good platform for such experiments due to the possibility of greatly enhanced nonlinear properties. Of particular interest are glasses infused with gold or silver nanoparticles [33, 36, 41], organic metamaterials [18], and polymers [54]. Beside metamaterials, a promising class of tunable and nonlinear systems include various saturating objects or materials, such as quantum wells [2, 91, 75, 99, 100], two-level systems [7], and dipolar fluids [89].

The existing research into fluctuating nonlinear systems is sparse, especially for the electromagnetic field. Early attempts concentrate on nonlinear Langevin equations $[96,95,49]$, yielding modified FDT relations. Nonlinearities are also encountered in interacting field theories $[21,40]$, which are applied in the setting of critical Casimir forces [50, 35]. Macroscopic quantum electrodynamics, probabilistic by nature, has been extended for nonlinear dielectrics $[27,37,79]$, but the theory lacks the versatility of FE and has not yet been applied to the Casimir effect.

Regarding fluctuational forces in nonlinear systems, there have been studies of the van der Waals force between nonlinear polarizable particles [55, 61, 84]. From a fieldtheoretical perspective, fluctuations near nonlinear boundary conditions [32], as well as the Casimir force in the presence of a nonlinear medium [45], have been considered.

Heat radiation of nonlinear systems is even less explored. The only applications con- 
cern optical cavities, which are laser resonator setups built around a nonlinear crystal. Refs. [43, 44], using coupled mode theory (essentially a Langevin equation), obtain a modified FDT relation similar to Refs. [96, 95, 49] as well as modified heat radiation spectra. It is not clear, however, how to generalize these results beyond the specific setup of an optical resonator.

To the author's knowledge, no analogue to FE exists in scientific literature for nonlinear materials besides Refs. [83, 85, 86].

\subsection{Thesis outline}

As the foundation for the rest of the thesis, the objective of Chapter 2 is to introduce the formalism of fluctuational electrodynamics and nonlinear optics necessary for later chapters. We start from the macroscopic Maxwell's equations, introduce the fluctuation-dissipation theorem (FDT), and obtain the linear stochastic Helmholtz equation. After a short introduction into the mathematics of nonlinear optics, the nonlinear polarizability will be added to the wave equation, resulting in a so-called stochastic nonlinear Helmholtz equation (SNHE). We demonstrate further how the average electric field obeys a similar (but deterministic) effective nonlinear Helmholtz equation (ENHE). The chapter is concluded by applying a basic perturbative analysis, leading to simpler forms of the SNHE and ENHE, which will be applied in specific cases over the course of the subsequent three chapters: in equilibrium (Chapter 3), in systems out of thermal equilibrium (Chapter 4), and in systems with a strong external field (Chapter 5).

Chapter 3 takes the equations obtained at the end of Chapter 2, the SNHE and ENHE, and evaluates them in equilibrium with the help of the FDT. We determine both the equilibrium noise currents and fluctuations and show that our use of the FDT is in agreement with the so-called Rytov theory of Ref. [58]. Using these, we determine the effective dielectric function or electromagnetic potential, which governs the behavior of the average electromagnetic field (and therefore linear response and optics). We use these results in a practical example: to calculate the force between two semi-infinite parallel plates, also known as the Casimir force.

In Chapter 4 , the system is taken out of equilibrium by assigning the noise in different objects different temperatures, but leaving it otherwise unchanged. This is called the local thermal equilibrium approximation: the temperatures are equalized locally (within objects) but not globally (between objects). This gives a robust way of calculating both the non-equilibrium fluctuations and heat transfer between the objects. The changing fluctuations also affect the effective dielectric function, with the interesting consequence that it is possible for a passive material to start behaving like a gain 


\section{Introduction}

medium. The effective dielectric function is used to give an explicit formula for the heat radiation a single nonlinear nanosphere. In the extreme case where the sphere has no absorption in equilibrium, we find that the net heat flow will always be away from the sphere, even if it is colder than the environment.

Adding strong external forces into the system in Chapter 5, we analyze how many degrees of freedom does the FDT remove from a simple scalar model (as opposed to full electromagnetic theory) without any further assumptions. We will see that without extra information (for example from a microscopic theory) or assumptions, the behavior of noise, and therefore field fluctuations, can not be uniquely determined. Nevertheless, the noise-fluctuation dualism offers a useful framework to apply and study further assumptions. The simplest case is also applied to calculate the field dependence of the fluctuations in a strong external field.

We conclude in Chapter 6 with a short summary of results and open questions, as well as an outlook to evaluate where and how the results of this thesis could be applied or developed further.

\section{Regarding work carried out before doctoral studies}

This project began during my Master's studies, so there is some seeming overlap with my previous thesis [83] in Chapter 3. However, the first paper on the subject [85], published more than half a year later, includes new numerical results and insights. The derivation for equilibrium was furthermore reworked, with the introduction of effective quantities, in Ref. [86]. Therefore a full account of the topic is given here, as it also forms the basis for later chapters. 


\section{Combining electromagnetic fluctuations and nonlinear optics}

The objective of this chapter is to introduce the general framework and mathematical apparatus of both fluctuational electrodynamics and nonlinear optics. This will set a starting point for the rest of the thesis, where specific aspects of the combination of these theories are presented and explored.

The first section covers basic macroscopic electromagnetic theory and the fluctuationdissipation theorem (FDT), thus laying out the formalism of linear fluctuational electrodynamics, while Section 2.2 introduces nonlinear optics.

In the second half of the chapter, these ideas are combined into nonlinear fluctuational electrodynamics. In Section 2.3, the noise is formally added to the equations of nonlinear electrodynamics generally. A perturbative expansion in the nonlinear susceptibility is then made in Section 2.4. We obtain equations of motion for the stochastic electromagnetic field, however only in terms of as yet unknown noise. The lack of predictive power will be addressed later in equilibrium (Chapter 3), for objects with different temperatures (Chapter 4 ), and in the presence of an external field (Chapter 5).

\subsection{From Maxwell's equations to linear fluctuational electrodynamics}

\subsubsection{The wave equation in Fourier space}

The well-known Maxwell's equations give a microscopic description of the electromagnetic field. They relate the electric $(\mathbf{E})$ and magnetic $(\mathbf{B})$ fields to charges $(\rho)$ and currents (J) [38].

$$
\begin{aligned}
\nabla \times \mathbf{E}(\mathbf{r}, t) & =-\partial_{t} \mathbf{B}(\mathbf{r}, t) \\
\nabla \times \mathbf{B}(\mathbf{r}, t) & =\varepsilon_{0} \mu_{0} \partial_{t} \mathbf{E}(\mathbf{r}, t)+\mu_{0} \mathbf{J}(\mathbf{r}, t) \\
\nabla \cdot \mathbf{E}(\mathbf{r}, t) & =\frac{\rho(\mathbf{r}, t)}{\varepsilon_{0}} \\
\nabla \cdot \mathbf{B}(\mathbf{r}, t) & =0
\end{aligned}
$$




\section{Combining electromagnetic fluctuations and nonlinear optics}

where $\partial_{t} \equiv \frac{\partial}{\partial t}$. Note that we are using SI units throughout the thesis, so $\varepsilon_{0}$ and $\mu_{0}$ are the permittivity and permeability of free space, respectively. $\mathbf{r}$ is the spatial coordinate and $t$ time, as usual. We will only write these arguments explicitly, if necessary for clarity, but leave them out otherwise for brevity.

The beauty of these equations is that they are exact, if it is possible to take all the charges and currents into account, until quantum effects become important. This is too much detail for everyday applications involving matter interacting with the electromagnetic field (such as light refracting through glass), however, because the number of individual charges is of the order of $10^{24}$ (number molecules in one mole). In most cases it is possible to coarse grain (average) the interaction with matter, replacing the bound, microscopic charges by so-called auxiliary fields, which correspond to the electromagnetic field generated by matter in response to outside field. The fields then obey the so-called macroscopic Maxwell's equations [67],

$$
\begin{aligned}
\nabla \times \mathbf{E} & =-\partial_{t} \mathbf{B}, \\
\nabla \times \mathbf{H} & =\partial_{t} \mathbf{D}+\mathbf{J}_{f}, \\
\nabla \cdot \mathbf{D} & =\rho_{f}, \\
\nabla \cdot \mathbf{B} & =0,
\end{aligned}
$$

where $\mathbf{J}_{f}$ and $\rho_{f}$ are the free (as opposed to bound) current and charge densities, respectively. The auxiliary fields $\mathbf{D}$ and $\mathbf{H}$ are defined through the polarization, $\mathbf{P}=\mathbf{P}(\mathbf{E})$, and magnetization, $\mathbf{M}=(\mathbf{B})$, as follows:

$$
\begin{aligned}
& \mathbf{D}(\mathbf{r}, t)=\varepsilon_{0} \mathbf{E}(\mathbf{r}, t)+\mathbf{P}(\mathbf{r}, t), \\
& \mathbf{H}(\mathbf{r}, t)=\frac{1}{\mu_{0}} \mathbf{B}(\mathbf{r}, t)-\mathbf{M}(\mathbf{r}, t) .
\end{aligned}
$$

Throughout the thesis we will deal with nonmagnetic materials, so we can set $\mathbf{M}=0$. One can then obtain the following equation for the electric field,

$$
\nabla \times \nabla \times \mathbf{E}+\mu_{0} \varepsilon_{0} \partial_{t}^{2} \mathbf{E}=-\mu_{0} \partial_{t}^{2} \mathbf{P}-\mu_{0} \partial_{t} \mathbf{J}_{f}
$$

This is the electromagnetic wave equation or Helmholtz equation ${ }^{1}$, which will be the main object of study in this thesis.

\footnotetext{
${ }^{1}$ A vector Helmholtz equation conventionally has a Laplacian instead of a double curl, while the latter is often referred to by the same name [72]. We only use the double curl version in this thesis so there should not be any confusion.
} 


\section{Fourier space}

It is much more convenient to treat Eq. (2.11) in frequency space. That is because the differential operators in time transform into multiplications by frequency. Therefore, instead of a partial differential equation in time, we have a system of equations in frequencies. This is especially useful in the linear case, because the equations for different frequencies are not coupled and can be solved individually.

We will use the Fourier transform in the same form as Ref. [67],

$$
\begin{aligned}
& f(t)=\int_{-\infty}^{\infty} \mathrm{d} \omega e^{-i \omega t} \hat{f}(\omega), \\
& \hat{f}(\omega)=\frac{1}{2 \pi} \int_{-\infty}^{\infty} \mathrm{d} t e^{i \omega t} f(t) .
\end{aligned}
$$

The spectral representation of the Helmholtz equation is then

$$
\nabla \times \nabla \times \mathbf{E}(\mathbf{r}, \omega)-\frac{\omega^{2}}{c^{2}} \mathbf{E}(\mathbf{r}, \omega)=\frac{1}{\varepsilon_{0}} \frac{\omega^{2}}{c^{2}} \mathbf{P}(\mathbf{r}, \omega)+i \omega \mu_{0} \mathbf{J}_{f}(\mathbf{r}, \omega),
$$

where we used the speed of light in vacuum, $c=\sqrt{\frac{1}{\varepsilon_{0} \mu_{0}}}$. We use the same symbols for spectral and time-domain fields, because time and frequency domain quantities do not appear in the same equation. We will also be using the spectral representation exclusively in the rest of the thesis and thus leave out the frequency argument, if it is clear from context.

\section{Constitutive relations - linear case}

The constitutive relations describe how a material responds to the electromagnetic field, giving the polarization and magnetization field as functions of the electric and magnetic field respectively. Since we work with non-magnetic materials, we only need $\mathbf{P}(\mathbf{E})$.

In local linear materials ${ }^{2}$ this is given as

$$
P_{i}(\mathbf{r}, \omega)=\varepsilon_{0} \chi_{i j}^{(1)}(\mathbf{r} ;-\omega, \omega) E_{j}(\mathbf{r}, \omega),
$$

where $\chi_{i j}^{(1)}(\mathbf{r} ;-\omega, \omega)$ is called the linear susceptibility and summation is implied over repeated indices (also known as the Einstein summation). It depends on $\mathbf{r}$ even for

\footnotetext{
${ }^{2}$ These are materials where the polarization $\mathbf{P}(\mathbf{r})$ depends linearly on the electric field at the same position $\mathbf{r}$ at times $t^{\prime} \leq t$. More generally it could have a nonlinear dependence on the field, leading to nonlinear optics (see Section 2.2), or on the field at different points $\mathbf{r}^{\prime} \neq \mathbf{r}$, which we shall not address.
} 


\section{Combining electromagnetic fluctuations and nonlinear optics}

homogeneous objects, in which case it is a step function across the boundary. Similar to fields, we leave the explicit arguments out unless necessary.

\subsubsection{Linear Helmholtz equation in operator notation}

\section{Operator notation and the free Green's function}

Operators, written in blackboard script, are similar to vector fields. As an example, they can be an outer (or tensor) product of two vector fields,

$$
\mathbb{O}_{i j}\left(\mathbf{r}, \mathbf{r}^{\prime}\right)=\mathbf{A}_{i}(\mathbf{r}) \otimes \mathbf{B}_{j}\left(\mathbf{r}^{\prime}\right) .
$$

When written next to a vector field, the operator is applied by summing/integrating over corresponding indices/coordinates, giving a new vector field.

$$
\mathbb{O} \mathbf{A}=\mathbf{B} \Longleftrightarrow \mathbf{B}_{i}(\mathbf{r})=\mathbb{O}_{i j}\left(\mathbf{r}, \mathbf{r}^{\prime}\right) \mathbf{A}_{j}\left(\mathbf{r}^{\prime}\right) \text {. }
$$

Similarly, an operator can be applied on another operator, resulting in a new operator. This allows for much clearer notation, especially when more than a few operators need to be applied together.

One example of an operator we use a lot is the dyadic electromagnetic Green's function in free space, $\mathbb{G}_{0}$. It is the solution to the equation

$$
\nabla \times \nabla \times \mathbb{G}_{0}-\frac{\omega^{2}}{c^{2}} \mathbb{G}_{0}=\mathbb{I},
$$

where the identity operator $\mathbb{I}=\delta_{i j} \delta^{(3)}\left(\mathbf{r}-\mathbf{r}^{\prime}\right)$ is on the right hand side. The solution to this equation can be written explicitly as [72]

$$
\mathbb{G}_{0}\left(\omega ; \mathbf{r}, \mathbf{r}^{\prime}\right)=\left(\mathbb{I}-\frac{c^{2}}{\omega^{2}} \nabla_{\mathbf{r}} \otimes \nabla_{\mathbf{r}^{\prime}}\right) \frac{e^{i \frac{\omega}{c}\left|\mathbf{r}-\mathbf{r}^{\prime}\right|}}{4 \pi\left|\mathbf{r}-\mathbf{r}^{\prime}\right|} .
$$

Even though we do not need the explicit form of $\mathbb{G}_{0}$, it is useful, because it behaves as an inverse to the free Helmholtz operator,

$$
\mathbb{H}_{0}=\nabla \times \nabla \times-\frac{\omega^{2}}{c^{2}} \mathbb{I} .
$$

By introducing also the dielectric potential operator $[72,52]$ (we will mostly call it just the "potential")

$$
\mathbb{V}_{i j}\left(\mathbf{r}, \mathbf{r}^{\prime}, \omega\right)=\frac{\omega^{2}}{c^{2}} \chi_{i j}^{(1)}(\mathbf{r} ;-\omega, \omega) \delta\left(\mathbf{r}-\mathbf{r}^{\prime}\right)
$$


we can rewrite the Helmholtz equation [Eq. (2.14)] as

$$
\left(\mathbb{H}_{0}-\mathbb{V}\right) \mathbf{E}=i \omega \mu_{0} \mathbf{J}_{f} .
$$

\section{The incoming field and the deterministic linear Helmholtz equation}

Using the dyadic Green's function,

$$
\mathbb{G}=\left(\mathbb{H}_{0}-\mathbb{V}\right)^{-1},
$$

the solution to Eq 2.22 can be written symbolically as

$$
\mathbf{E}=\mathbf{E}_{0}+i \omega \mu_{0} \mathbb{G} \mathbf{J}_{f}
$$

where $\mathbf{E}_{0}$ is the homogeneous $\left(\mathbf{J}_{f}=0\right)$ solution. The second term represents then the fields generated by the sources $\mathbf{J}_{f}$ and scattered by the potential $\mathbb{V}$.

If the sources are very far away from any objects, they can be represented as an incoming field,

$$
\mathbf{E}_{\mathrm{in}}=i \omega \mu_{0} \mathbb{G}_{0} \mathbf{J}_{f} .
$$

These are sources that generate the field $\mathbf{E}_{\text {in }}$ in vacuum. The scattered field is then

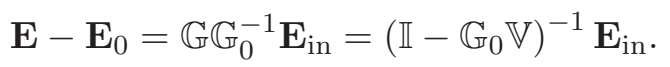

The inverse operator $\left(\mathbb{I}-\mathbb{G}_{0} \mathbb{V}\right)^{-1}$ can be expanded to yield the Lippmann-Schwinger series [60],

$$
\mathbf{E}-\mathbf{E}_{0}=\mathbf{E}_{\text {in }}+\mathbb{G}_{0} \mathbb{V} \mathbf{E}_{\text {in }}+\mathbb{G}_{0} \mathbb{V} \mathbb{G}_{0} \mathbb{V} \mathbf{E}_{\text {in }}+\ldots
$$

Each successive element in the series represents a term with higher order scattering. This can be used to approximate the full Green's function, if the scattering cross-section is low.

With the addition of the incoming field, we arrive at the final form of the deterministic linear Helmholtz equation [Eq. 2.22],

$$
\left(\mathbb{H}_{0}-\mathbb{V}\right) \mathbf{E}=\mathbb{H}_{0} \mathbf{E}_{\text {in }} .
$$

This represents the relation between the total electric field $\mathbf{E}$ and the incoming or external field $\mathbf{E}_{\text {in }}$. 


\section{The linear response}

Now we can calculate formally the (in this case fully linear) response of the field $\mathbf{E}$ to the incoming field $\mathbf{E}_{\mathrm{in}}$. This is most clearly defined through a functional derivative (see Appendix (A)). The electric field (later, its average) is a function of an incoming field, $\mathbf{E}=\mathbf{E}\left(\mathbf{E}_{\text {in }}\right)$ (for example as per Eq. 2.28). The derivative of $\mathbf{E}$ with respect to $\mathbf{E}_{\text {in }}$ is $\mathbf{A}=\frac{\delta \mathbf{E}}{\delta \mathbf{E}_{\text {in }}}$, such that

$$
\mathbf{A}(\mathbf{f})=\lim _{\epsilon \rightarrow 0} \frac{\mathbf{E}\left(\mathbf{E}_{\text {in }}\right)-\mathbf{E}\left(\mathbf{E}_{\text {in }}+\epsilon \mathbf{f}\right)}{\epsilon} \forall \mathbf{f} .
$$

From Eq. (2.26), we see that it is actually the linear operator $\mathbb{G}_{0}^{-1}$. This can be seen by taking the derivative $\delta_{\mathbf{E}_{\text {in }}}$ of both sides of the Helmholtz equation [Eq. (2.28)],

$$
\begin{gathered}
\delta_{\mathbf{E}_{\text {in }}}\left[\left(\mathbb{H}_{0}-\mathbb{V}\right) \mathbf{E}\right]=\delta_{\mathbf{E}_{\text {in }}}\left[\mathbb{H}_{0} \mathbf{E}_{\text {in }}\right] \\
\left(\mathbb{H}_{0}-\mathbb{V}\right) \delta_{\mathbf{E}_{\text {in }}} \mathbf{E}=\mathbb{H}_{0} \underbrace{\delta_{\mathbf{E}_{\text {in }}} \mathbf{E}_{\text {in }}}_{=\mathbb{I}} . \\
\text { Multiply from left by } \mathbb{G}=\left(\mathbb{H}_{0}-\mathbb{V}\right)^{-1}: \\
\delta_{\mathbf{E}_{\text {in }}} \mathbf{E}=\mathbb{G} \mathbb{H}_{0}=\mathbb{G} \mathbb{G}_{0}^{-1} .
\end{gathered}
$$

These manipulations are compact, but hide a lot of very tedious mathematics, especially when treating nonlinear equations further in the thesis. In order to save space, this formalism shall be used extensively.

\subsubsection{Fluctuations of the electromagnetic field}

\section{Fluctuations and ensemble averaging}

The electromagnetic field is not deterministic, but rather it exhibits both quantum and thermal fluctuations. This means that instead of a specific solution for the field $\mathbf{E}$ in Eq. (2.28), a whole ensemble of fields need to be considered, with each possible field having probability of being realized. Therefore, we need to distinguish between a particular realization $\mathbf{E}$ of a field and its average value $\langle\mathbf{E}\rangle$, with the fluctuations (from mean) defined as

$$
\delta \mathbf{E}=\mathbf{E}-\langle\mathbf{E}\rangle .
$$

In particular, we consider here the ensemble average (rather than time average). In equilibrium, for example, the realizations are weighed by the difference of their energy $\mathcal{H}[\mathbf{E}]$ from the free energy $F=\langle\mathcal{H}[\mathbf{E}]\rangle[1]$,

$$
\langle\mathbf{E}\rangle^{\mathrm{eq}}=\frac{1}{\mathcal{Z}} \int \mathcal{D} e^{\frac{F-\mathcal{H}[\mathbf{E}]}{k_{\mathrm{B}} T}} \mathbf{E} .
$$


Here $\int \mathcal{D}$ represents integration over all possible realizations of $\mathbf{E}, \mathcal{Z}=\int \mathcal{D} e^{\frac{F-\hat{H}}{k_{\mathrm{B}} T}}$ is the partition function, $T$ is the temperature, and $k_{\mathrm{B}}$ is the Boltzmann constant. This includes quantum effects, if discrete energy levels in the Hamiltonian $\mathcal{H}$ are taken into account.

In order to describe the electromagnetic field in this way, a correct Hamiltonian needs to be defined in order to obtain the macroscopic Maxwell's equations (2.7). This is a feasible approach to fluctuational electrodynamics, especially to calculate Casimir force as a derivative of the free energy with respect to distance as in Ref. [72]. For other applications, especially to extend the theory out of equilibrium for heat transfer calculations, it is more convenient to work directly with fields (as in Refs. [52,51] and indeed in the current work).

\section{Fluctuation-dissipation theorem for the electromagnetic field}

An extremely important result, which is can be proven from the form of the ensemble average $[53,1,29]$, is the fluctuation-dissipation theorem (FDT), which relates the strength of the fluctuations in equilibrium to the linear response of the average field close to equilibrium. In units used in this thesis, it can be written as

$$
\left\langle\delta \mathbf{E}_{\omega} \otimes \delta \mathbf{E}_{\omega^{\prime}}^{*}\right\rangle^{\mathrm{eq}}=\delta\left(\omega-\omega^{\prime}\right) \frac{b(\omega)}{2 i} \tilde{\mathbb{G}}_{\mathrm{AH}} .
$$

$\widetilde{\mathbb{G}}_{\mathrm{AH}}=\tilde{\mathbb{G}}-\widetilde{\mathbb{G}}^{\dagger}$ is the anti-Hermitian part $^{3}$ of the linear response (a tilde is used to denote that this can be different from the Green's function $\left.\mathbb{G}=\left(\mathbb{H}_{0}-\mathbb{V}\right)^{-1}\right)$,

$$
\tilde{\mathbb{G}}=\lim _{\mathbf{E}_{\text {in }} \rightarrow 0} \frac{\delta\langle\mathbf{E}\rangle}{\delta \mathbf{E}_{\text {in }}} \mathbb{G}_{0} \text {. }
$$

For a symmetric response (always true for linear systems [29]), the anti-Hermitian part is equivalent to the imaginary part $\frac{1}{2 i} \tilde{\mathbb{G}}_{\mathrm{AH}}=\operatorname{Im} \tilde{\mathbb{G}}$. This is can be viewed as the absorption in the system. Since the fluctuating currents on the left hand side of Eq. (2.36) represent emission, the FDT in the electromagnetic case is a form of the Kirchhoff's law of radiation.

Temperature $T$ is included in Eq. (2.36) through the coefficient

$$
b(\omega)=\frac{\hbar}{\pi \varepsilon_{0}} \frac{\omega^{2}}{c^{2}} \frac{1}{1-e^{-\frac{\hbar \omega}{k_{\mathrm{B}} T}}},
$$

\footnotetext{
${ }^{3}$ The "dagger" represents Hermitian conjugation, $\mathbb{O}_{i j}^{\dagger}\left(\mathbf{r}, \mathbf{r}^{\prime}\right)=\mathbb{O}_{j i}^{*}\left(\mathbf{r}^{\prime}, \mathbf{r}\right)$.
} 


\section{Combining electromagnetic fluctuations and nonlinear optics}

where beside familiar constants the reduced Planck constant $\hbar$ appears, denoting the inclusion of quantum fluctuations. Depending on literature source, a different coefficient

$$
a(\omega)=b(\omega)-b(-\omega)=\frac{\hbar}{\pi \varepsilon_{0}} \frac{\omega^{2}}{c^{2}} \operatorname{coth}\left(\frac{\hbar \omega}{2 k_{\mathrm{B}} T}\right),
$$

might appear (e.g. in [52]). This is dependent on how the negative frequency components are treated. In this thesis, generally the negative frequencies are included in $\omega$-integrals, so $b(\omega)$ as defined Eq. (2.38) will be used more often.

The significance of this theorem as a cornerstone of fluctuational electrodynamics cannot be overstated: the second moment of the equilibrium fluctuations can be determined purely by the response of the average field to an infinitesimal probing field $\mathbf{E}_{\text {in }}$. Since the former gives the Casimir force and (out of equilibrium) radiative heat transfer, two easily measurable effects, either side of Eq. (2.36) (the FDT) represents the results of two different experiments. In other words, it is the backbone that enables the prediction of the Casimir force and heat radiation based purely on the measured dielectric properties of the objects.

On the other hand, the FDT does not give a full description of the stochastic quantity $\delta$ E. First, it is only valid strictly in equilibrium, whereas out of equilibrium properties need to be obtained with clever extensions or approximations (e.g. in Chapter 4 a local equilibrium, but global non-equilibrium, is assumed). Second, the first $(\langle\delta \mathbf{E}\rangle=0$ by definition) and second moments of a fluctuating quantity determine the stochastic variable only if it is Gaussian and therefore Isserlis' theorem ${ }^{4}$ can be used to express any moment in terms of second moments. In the general case, the higher moments can be independent. Since the probability distribution of a stochastic variable is represented as a series of these higher moments ${ }^{5}$, the FDT is not enough to determine this.

\section{Fluctuations induced by random sources in linear systems}

The Helmholtz equation in Eq. (2.28) does not support thermal and quantum fluctuations, if the incoming field is deterministic. This can be seen easily by taking the average and separating the equations for the average field $\overline{\mathbf{E}}=\langle\mathbf{E}\rangle$ and the fluctuations

\footnotetext{
${ }^{4}$ It is also known as Wick's theorem or the Furutsu-Novikov formula depending on the area of physics or mathematics.

${ }^{5}$ This is called a Kramers-Moyal expansion. It is a general form of the Focker-Planck equation, which only includes the first and second moments from the series.
} 
$\delta \mathbf{E}=\mathbf{E}-\langle\mathbf{E}\rangle:$

$$
\begin{aligned}
\left(\mathbb{H}_{0}-\mathbb{V}\right) \mathbf{E} & =\mathbb{H}_{0} \mathbf{E}_{\mathrm{in}}, \\
\left(\mathbb{H}_{0}-\mathbb{V}\right) \overline{\mathbf{E}} & =\mathbb{H}_{0} \mathbf{E}_{\mathrm{in}}, \\
\left(\mathbb{H}_{0}-\mathbb{V}\right) \delta \mathbf{E} & =0 .
\end{aligned}
$$

The solution is $\mathbf{E}=\overline{\mathbf{E}}, \delta \mathbf{E}=0$. This could be expected, since Eq. (2.28) contains only deterministic sources and coefficients.

The problem is that the above equations are in conflict with physical reality, the existence of fluctuations can be easily measured. Mathematically, since the linear response is nonzero, $\lim _{\mathbf{E}_{\text {in }} \rightarrow 0} \frac{\delta \overline{\mathbf{E}}}{\delta \mathbf{E}_{\text {in }}} \mathbb{G}_{0}=\left(\mathbb{H}_{0}-\mathbb{V}\right)^{-1}$, without fluctuations the FDT from Eq. (2.36) is not fulfilled. In the theory of Langevin equations, the solution is to add a random force or noise to the deterministic equation. More generally, they are also called the "fictitious forces" [1]. In the electromagnetic case, the natural way is to include random sources into Eq. (2.28), which are called Rytov currents [58].

As per Eq. (2.22), we will include the Rytov currents as

$$
i \omega \mu_{0} \mathbf{J}_{\text {Rytov }}=\mathbf{F} \text {. }
$$

We shall use the terms "Rytov currents" and "noise" interchangeably to refer to either $\mathbf{J}_{\text {Rytov }}$ or $\mathbf{F}$. The stochastic Helmholtz equation can now be written as

$$
\left(\mathbb{H}_{0}-\mathbb{V}\right) \mathbf{E}=\mathbb{H}_{0} \mathbf{E}_{\text {in }}+\mathbf{F} \text {. }
$$

The noise must be chosen such that the FDT holds, but otherwise it has no restrictions. Separating again the equation for $\overline{\mathbf{E}}$ and $\delta \mathbf{E}$, we get simply

$$
\begin{aligned}
\left(\mathbb{H}_{0}-\mathbb{V}\right) \overline{\mathbf{E}} & =\mathbb{H}_{0} \mathbf{E}_{\mathrm{in}}, \\
\left(\mathbb{H}_{0}-\mathbb{V}\right) \delta \mathbf{E} & =\mathbf{F} .
\end{aligned}
$$

This is a consequence of the superposition principle in linear systems: the noise $\mathbf{F}$ is a source for the fluctuations $\delta \mathbf{E}$, while the deterministic sources (the incoming field) create an average field. So the fluctuations $\delta \mathbf{E}$ and $\overline{\mathbf{E}}$ are uncoupled. This means that, conveniently, we can represent the total field correlator in linear systems as

$$
\left\langle\mathbf{E} \otimes \mathbf{E}^{*}\right\rangle=\left\langle\delta \mathbf{E} \otimes \delta \mathbf{E}^{*}\right\rangle^{\mathrm{eq}}+\overline{\mathbf{E}} \otimes \overline{\mathbf{E}}^{*} .
$$

This has a consequence for the electromagnetic pressure (see Appendix C) in linear systems: it is simply the sum of the Casimir effect in equilibrium and pressure due to the deterministic average field. We will see that in the nonlinear case the situation be- 


\section{Combining electromagnetic fluctuations and nonlinear optics}

comes more complicated, largely because the fluctuations and the average field become coupled.

Because of the superposition principle, adding noise to a linear system has no effect on the linear response,

$$
\tilde{\mathbb{G}}_{\text {lin }}=\lim _{\mathbf{E}_{\text {in }} \rightarrow 0} \frac{\delta\langle\mathbf{E}\rangle}{\delta \mathbf{E}_{\text {in }}} \mathbb{G}_{0}=\left(\mathbb{H}_{0}-\mathbb{V}\right)^{-1} .
$$

Taking the average of Eq. (2.46) gives the mean of the Rytov currents,

$$
\langle\mathbf{F}\rangle=\left(\mathbb{H}_{0}-\mathbb{V}\right)\langle\delta \mathbf{E}\rangle=0,
$$

and the (equilibrium) second moment of $\mathbf{F}$ can be obtained directly from Eqs. (2.36) and 2.46,

$$
\begin{aligned}
\left\langle\mathbf{F}_{\omega} \otimes \mathbf{F}_{\omega^{\prime}}^{*}\right\rangle^{\mathrm{eq}} & =\left\langle\left[\left(\mathbb{H}_{0}-\mathbb{V}\right) \delta \mathbf{E}\right]_{\omega} \otimes\left[\left(\mathbb{H}_{0}-\mathbb{V}\right) \delta \mathbf{E}\right]_{\omega^{\prime}}^{*}\right\rangle^{\mathrm{eq}} \\
& =\left(\mathbb{H}_{0}-\mathbb{V}\right)\left\langle[\delta \mathbf{E}]_{\omega} \otimes \delta \mathbf{E}_{\omega^{\prime}}^{*}\right)^{\mathrm{eq}}\left(\mathbb{H}_{0}-\mathbb{V}\right)^{\dagger} \\
& =-\delta\left(\omega-\omega^{\prime}\right) b(\omega) \operatorname{Im}\left(\mathbb{H}_{0}-\mathbb{V}\right),
\end{aligned}
$$

where we used the fact that in equilibrium $\mathbb{V}$ is symmetric. Note that this correlator is completely local (see the discussion at the end of Section (3.1.2)), therefore Rytov currents at different points in space must be uncorrelated. The noise at any point is given directly by the imaginary part of the dielectric function, the absorption. Since in linear systems the fluctuations and therefore noise can be assumed to be Gaussian [29], Eqs. (2.49) and (2.50) give a complete description of the Rytov currents $\mathbf{F}$ in equilibrium.

One might wonder, what is the purpose of all the trouble of adding noise to the Helmholtz equation and calculating its first and second moments if all the interesting (and measurable) effects are already contained in the fluctuations $\left\langle\delta \mathbf{E} \otimes \delta \mathbf{E}^{*}\right\rangle$, which we know from the FDT by Eq. (2.36) already. There are two big reasons.

First, by making the Helmholtz equation explicitly stochastic, it gives a more complete description of the fluctuating field. This will be important in the nonlinear case, where it is important to keep track of assumptions and keep the theory self-consistent.

Second, because of the locality of the Rytov currents and their semi-physical nature (they represent macroscopic currents), reasonable assumptions can be made to extend the theory out of equilibrium. In Chapter 4, for example, the temperature of these currents will be changed while keeping the statistics otherwise the same. This gives a very accurate description of heat radiation, otherwise unobtainable purely from the FDT, which remains valid in equilibrium only. 


\subsection{Nonlinear optics}

Nonlinear optics or electrodynamics is a very wide and well-studied field of physics, which encompasses many different effects and processes [13]. This leads to widely different approximations and theoretical approaches, especially if only a particular (type of) nonlinear effect is of interest.

Our goal is to include the physics of optically nonlinear materials as generally as feasible. In order not to complicate calculations unnecessarily, only local effects will be considered, just like in the linear case. Most prominently, this leaves out the nonlinear optical properties of plasmas, which are inherently non-local [93]. The extension of this work to non-local response is expected to be straightforward, if tedious.

\subsubsection{The nonlinear polarizability}

In order to describe processes beyond the linear Helmholtz equation, Eq. (2.28), which was obtained by assuming that the material responds purely linearly to the electromagnetic field in Eq. (2.15), nonlinear terms in the electric field must be included. The most straightforward, and physical, way to do that is to add the possibility for the material to respond in an arbitrary fashion to the electric field, not just linearly as per Eq. (2.15). In particular, the polarization field $\mathbf{P}$ in Eq. (2.9) will be allowed to depend in a general way on the electric field (leaving out linear and nonlinear magnetization).

\section{Time domain response functions as susceptibilities in Fourier space}

The polarization field $\mathbf{P}$ describes the field generated by a material as a response to the electric field $\mathbf{E}$. This response can be represented as a power series around $\mathbf{E}=$ 0 [13]. For "normal" field strengths, which shall be considered in this thesis, this series converges very fast (see Ref. [13] as well as the discussion in Section 2.4). 
2. Combining electromagnetic fluctuations and nonlinear optics

In time domain, the polarization field can be written in terms of the response functions ${ }^{6}$,

$$
\begin{aligned}
P_{i}(t) & =\varepsilon_{0} \sum_{n=1}^{\infty}\left[\prod_{m=1}^{n} \int_{-\infty}^{\infty} \mathrm{d} \tau_{m} E_{j_{m}}\left(t-\tau_{m}\right)\right] R_{i j_{1} . . j_{n}}^{(n)}\left(\tau_{1}, . ., \tau_{n}\right) \\
& =\varepsilon_{0} \int_{-\infty}^{\infty} \mathrm{d} \tau R_{i j}^{(1)}(\tau) E_{j}(t-\tau) \\
& +\varepsilon_{0} \int_{-\infty}^{\infty} \mathrm{d} \tau_{1} \int_{-\infty}^{\infty} \mathrm{d} \tau_{2} R_{i j_{1} j_{2}}^{(2)}\left(\tau_{1}, \tau_{2}\right) E_{j_{1}}\left(t-\tau_{1}\right) E_{j_{2}}\left(t-\tau_{2}\right) \\
& +\ldots
\end{aligned}
$$

Note that even though we integrate over positive and negative time differences $\tau$ (to facilitate the Fourier transform), causality requires that the fields only at times $t^{\prime}<t$ affect $\mathbf{P}(t)$, therefore

$$
R^{(n)}\left(\tau_{1}, . ., \tau_{n}\right) \propto \prod_{m=1}^{n} \Theta\left(\tau_{m}\right)
$$

The susceptibilities are defined as the Fourier components of the responses,

$$
\chi_{i j_{1} . . j_{n}}^{(n)}\left(-\omega_{\sigma} ; \omega_{1}, . ., \omega_{n}\right)=\left(\prod_{m=1}^{n} \int_{-\infty}^{\infty} \mathrm{d} \tau_{m} e^{i \omega_{m} \tau_{m}}\right) R_{i j_{1} . . j_{n}}^{(n)}\left(\tau_{1}, . ., \tau_{n}\right),
$$

where the "extra" argument is the sum of frequencies, $\omega_{\sigma}=\sum_{m=1}^{n} \omega_{m}$, and appears because the response functions only depend on time differences. This is included by convention and denotes the frequency of an incoming wave.

\section{Nonlinear polarization in frequency space}

Applying $\left(\prod_{m=1}^{n} \int_{-\infty}^{\infty} \mathrm{d} \omega_{m} e^{-i \omega_{m} \tau_{m}^{\prime}}\right)$ to both sides and using $\int_{-\infty}^{\infty} \mathrm{d} \omega e^{i \omega t}=2 \pi \delta(t)$ yields the inverse transform,

$$
R_{i j_{1} . . j_{n}}^{(n)}\left(\tau_{1}, . ., \tau_{n}\right)=(2 \pi)^{-n}\left(\prod_{m=1}^{n} \int_{-\infty}^{\infty} \mathrm{d} \omega_{m} e^{-i \omega_{m} \tau_{m}}\right) \chi_{i j_{1} . . j_{n}}^{(n)}\left(-\omega_{\sigma} ; \omega_{1}, . ., \omega_{n}\right)
$$

${ }^{6}$ The response functions $R_{i j_{1} . . j_{n}}^{(n)}\left(\tau_{1}, . ., \tau_{n}\right)$ are also known as memory kernels, since they show how much $P(t)$ depends on the electric field at times $t-\tau_{n}$. It represents therefore the "memory" of the material. 
Note that the $(2 \pi)^{-n}$ is in the inverse Fourier transform, opposite from Eqs. (2.12) and (2.13). This is necessary to end up with the correct equation in frequency space. Eq. (2.51) can now be transformed,

$$
P_{i}(\omega)=\varepsilon_{0} \sum_{n=1}^{\infty}\left[\prod_{m=1}^{n} \int_{-\infty}^{\infty} \mathrm{d} \omega_{m} E_{j_{m}}\left(\omega_{m}\right)\right] \chi_{i j_{1} . . j_{n}}^{(n)}\left(-\omega_{\sigma} ; \omega_{1}, . ., \omega_{n}\right) \delta\left(\omega_{\sigma}-\omega\right),
$$

which is the nonlinear counterpart of Eq. (2.15), as can be seen from the first few terms,

$$
\begin{aligned}
P_{i}(\omega) & =\varepsilon_{0} E_{j}(\omega) \chi_{i j}^{(1)}(-\omega ; \omega) \\
& +\varepsilon_{0} \int_{-\infty}^{\infty} \mathrm{d} \omega_{1} \int_{-\infty}^{\infty} \mathrm{d} \omega_{2} \delta\left(\omega_{\sigma}-\omega\right) \chi_{i j_{1} j_{2}}^{(2)}\left(-\omega ; \omega_{1}, \omega_{2}\right) E_{j_{1}}\left(\omega_{1}\right) E_{j_{2}}\left(\omega_{2}\right) \\
& +\ldots
\end{aligned}
$$

Therefore all (local) nonlinear material properties are taken into account with the higher order susceptibilities.

\subsubsection{Properties of the nonlinear susceptibilities}

Before using the nonlinear polarizability to define a nonlinear Helmholtz equation, it is useful to discuss some properties of the nonlinear susceptibilities. These open up some very significant simplifications and approximations later in the thesis.

It turns out that the components of the susceptibilities $\chi_{i j_{1} . . j_{n}}^{(n)}\left(-\omega_{\sigma} ; \omega_{1}, . ., \omega_{n}\right)$ defined above are not completely independent. Rather, they must adhere to certain symmetries as a result of how they are defined and what they represent physically. These are very well covered in Ref. [13] and a quick overview is given here.

\section{Intrinsic symmetry}

The most basic symmetry, holding for the susceptibilities and responses of any material, stems from their definition in Eqs. (2.51) and (2.55) through a single field which commutes with itself. By rearranging the field terms and changing the integration/summation variables, it is clear that any pair of index-frequency pairs $\left(j_{a}, \omega_{a}\right)$ can be exchanged simultaneously. This can also be seen as a consequence of the fact that the susceptibilities are in essence derivatives (see the end of Appendix A)

E.g for third order we have

$$
\chi_{i j k l}^{(3)}\left(-\omega_{\sigma}, \omega_{1}, \omega_{2}, \omega_{3}\right)=\chi_{i k j l}^{(3)}\left(-\omega_{\sigma}, \omega_{2}, \omega_{1}, \omega_{3}\right)=\chi_{i l k j}^{(3)}\left(-\omega_{\sigma}, \omega_{3}, \omega_{2}, \omega_{1}\right) .
$$




\section{Combining electromagnetic fluctuations and nonlinear optics}

Note that this does not apply to the very first pair of arguments $\left(i,-\omega_{\sigma}\right)$ unless the material is lossless, in which case the susceptibilities can be shown to be derivatives of the internal energy.

\section{Causality}

Causality is the principle that the polarization field $\mathbf{P}(t)$ can not depend on the values of the electric field at future times $t^{\prime}>t$. From Eq. (2.51), this sets the following condition on the response functions,

$$
R_{i j_{1} . j_{n}}^{(n)}\left(\tau_{1}, . ., \tau_{n}\right)=\prod_{m=1}^{n} \Theta\left(\tau_{n}\right)
$$

where the Heaviside functions are defined as $\Theta\left(\tau_{n}>0\right)=1, \Theta\left(\tau_{n}<0\right)=0$. The susceptibilities in Eq. (2.53) are therefore defined as integrals over positive times,

$$
\chi_{i j_{1} . . j_{n}}^{(n)}\left(-\omega_{\sigma} ; \omega_{1}, . ., \omega_{n}\right)=\left(\prod_{m=1}^{n} \int_{0}^{\infty} \mathrm{d} \tau_{m} e^{i \omega_{m} \tau_{m}}\right) R_{i j_{1} . . j_{n}}^{(n)}\left(\tau_{1}, . ., \tau_{n}\right) .
$$

By letting the frequencies be complex, it can be seen that the expression converges if $\operatorname{Im} \omega_{m} \geq 0$ for all $\omega_{m}$. The susceptibilities can therefore be extended into the upper complex plane. This is very useful in expressions with integrals over frequencies [e.g. the Lifshitz formula, Eq. (C.13)] as these can be performed over the positive imaginary axis instead (this is called Wick's rotation or Matsubara summation), which is often both theoretically and numerically easier as oscillating functions become decaying exponential functions instead.

Of special importance is the third susceptibility of the form $\chi_{i j k l}^{(3)}\left(-\omega ; \omega, \omega^{\prime},-\omega^{\prime}\right)$, because it appears in the effective Helmholtz equation (2.129). While it converges for $\operatorname{Im} \omega>0$, it diverges for all $\operatorname{Im} \omega^{\prime} \neq 0$. Therefore a Wick's rotation in $\omega^{\prime}$ is not possible for expressions with $\chi_{i j k l}^{(3)}\left(-\omega ; \omega, \omega^{\prime},-\omega^{\prime}\right)$ and the integration over $\omega^{\prime}$ has to be done over the real line, which can be computationally challenging.

\section{Reality of responses}

The responses $R_{i j_{1} . . j_{n}}^{(n)}\left(\tau_{1}, . ., \tau_{n}\right)$ relate real fields to real fields by Eq (2.51), so they have to be real as well. For susceptibilities, this has the consequence that the real part must be symmetric and imaginary part antisymmetric in frequencies,

$$
\chi_{i j_{1} . . j_{n}}^{(n)}\left(-\omega_{\sigma} ; \omega_{1}, . ., \omega_{n}\right)=\left[\chi_{i j_{1} . . j_{n}}^{(n)}\left(\omega_{\sigma} ;-\omega_{1}, . .,-\omega_{n}\right)\right]^{*} .
$$




\section{Spatial symmetries}

The symmetries of the medium (for example crystal group symmetries) are also reflected in the susceptibilities. For example, a wide range of materials are isotropic (full rotational symmetry). Of the 9 elements of the first order susceptibility tensor only three equal ones remain:

$$
\chi_{i j}^{(1)}=\chi^{(1)} \delta_{i j} .
$$

The second order susceptibility vanishes completely for isotropic materials, while the third order has three independent components:

$$
\chi_{i j k l}^{(3)}=\chi_{1122}^{(3)} \delta_{i j} \delta_{k l}+\chi_{1212}^{(3)} \delta_{i k} \delta_{j l}+\chi_{1221}^{(3)} \delta_{i l} \delta_{j k} .
$$

\subsubsection{The nonlinear Helmholtz equation}

With the nonlinear polarization vector defined in Fourier space through nonlinear susceptibilities [Eq. (2.55)], we are in a position to derive a nonlinear version of the deterministic Helmholtz equation [Eq. (2.28)]. Noise will be added in Sections 2.3 and 2.4.

\section{The nonlinear potential operators}

It is convenient to introduce operator notation for the nonlinear susceptibilities in Eq. (2.55), similar to $\mathbb{V}$ in Eq. (2.21). Since most of the thesis will consider nonlinear effects up to third order (with the exception of Sections 2.3 and 5.2), two new operators $\mathcal{M}$ and $\mathcal{N}$ will be introduced,

$$
\begin{aligned}
(\mathcal{M}[\mathbf{A} \otimes \mathbf{B}])_{i}\left(\mathbf{r}, \mathbf{r}^{\prime} ; \omega\right) & =\delta\left(\mathbf{r}-\mathbf{r}^{\prime}\right) \frac{\omega^{2}}{c^{2}} \int \mathrm{d} \omega_{1} \mathrm{~d} \omega_{2} \delta\left(\omega-\omega_{\sigma}\right) \\
& \times \chi_{i j k}^{(2)}\left(-\omega_{\sigma}, \omega_{1}, \omega_{2}\right) A_{j}\left(\mathbf{r}, \omega_{1}\right) B_{k}\left(\mathbf{r}, \omega_{2}\right) \\
(\mathcal{N}[\mathbf{A} \otimes \mathbf{B} \otimes \mathbf{C}])_{i}\left(\mathbf{r}, \mathbf{r}^{\prime} ; \omega\right) & =\delta\left(\mathbf{r}-\mathbf{r}^{\prime}\right) \frac{\omega^{2}}{c^{2}} \int \mathrm{d} \omega_{1} \mathrm{~d} \omega_{2} \mathrm{~d} \omega_{3} \delta\left(\omega-\omega_{\sigma}\right) \\
& \times \chi_{i j k l}^{(3)}\left(-\omega_{\sigma}, \omega_{1}, \omega_{2}, \omega_{3}\right) A_{j}\left(\mathbf{r}, \omega_{1}\right) B_{k}\left(\mathbf{r}, \omega_{2}\right) C_{l}\left(\mathbf{r}, \omega_{3}\right) .
\end{aligned}
$$

As can be seen, unlike $\mathbb{V}$, these operators map tensor fields onto vector fields. Like $\mathbb{V}$, these are local operators and also linear (in whole tensors, not the components of the outer product). These operators have two nice properties that will be useful in the rest of the thesis. 


\section{Combining electromagnetic fluctuations and nonlinear optics}

First, the intrinsic symmetries of the nonlinear susceptibilities carries over very simply,

$$
\begin{aligned}
\mathcal{M}[\mathbf{A} \otimes \mathbf{B}] & =\mathcal{M}[\mathbf{B} \otimes \mathbf{A}], \\
\mathcal{N}[\mathbf{A} \otimes \mathbf{B} \otimes \mathbf{C}] & =[\mathbf{B} \otimes \mathbf{A} \otimes \mathbf{C}]=\mathcal{N}[\mathbf{A} \otimes \mathbf{C} \otimes \mathbf{B}] .
\end{aligned}
$$

This means that they only operate on the symmetric part of the tensors.

Second, by applying these operators to lower rank tensors, they themselves increase in rank. For example, mathematically the entities

$$
\begin{aligned}
\mathcal{M}[\mathbf{A}] & \equiv \mathcal{M}[\mathbf{A} \otimes \cdot], \\
\mathcal{N}[\mathbf{A} \otimes \mathbf{B}] & \equiv \mathcal{N}[\mathbf{A} \otimes \mathbf{B} \otimes \cdot],
\end{aligned}
$$

are operators of the same type as $\mathbb{V}$, because they map vectors fields onto vector fields,

$$
\begin{aligned}
\mathcal{M}[\mathbf{A}] \mathbf{E} & \equiv \mathcal{M}[\mathbf{A} \otimes \mathbf{E}], \\
\mathcal{N}[\mathbf{A} \otimes \mathbf{B}] \mathbf{E} & \equiv \mathcal{N}[\mathbf{A} \otimes \mathbf{B} \otimes \mathbf{E}] .
\end{aligned}
$$

This notation style is admittedly unusual, but allows for very easy and clear manipulation throughout the thesis.

\section{The nonlinear Helmholtz equation and nonlinear response}

Using the nonlinear potential operators and stopping at the third order, the nonlinear variant of the Helmholtz equation (2.28) can be written as

$$
\left(\mathbb{H}_{0}-\mathbb{V}\right) \mathbf{E}-\mathcal{M}[\mathbf{E} \otimes \mathbf{E}]-\mathcal{N}[\mathbf{E} \otimes \mathbf{E} \otimes \mathbf{E}]=\mathbb{H}_{0} \mathbf{E}_{\text {in }} .
$$

If the susceptibilities $\chi^{(1,2,3)}$ are known, this equation gives a complete description of (local) nonlinear optics up to third order.

A big part of nonlinear optics research concerns itself with either predicting the nonlinear susceptibilities from theory or measuring them experimentally. We consider these as input to the theory of nonlinear fluctuational electrodynamics as developed in this thesis. And while modeling $\chi^{(2)}$ and $\chi^{(3)}$ is anything but easy, we can relate them directly to nonlinear scattering measurements, just like the linear potential $\mathbb{V}$ can be related to the linear scattering experiment by Eq. (2.26).

For example, by taking successive functional derivatives of Eq. (2.71) (see also Appendix A) with respect to $\mathbf{E}_{\text {in }}$, and recalling the symmetries of the nonlinear potential operators, the operator $\mathcal{M}$ (operator form of the second order susceptibility $\chi^{(2)}$ ) can 
be obtained,

$$
\begin{aligned}
\delta_{\mathbf{E}_{\text {in }}} \mathbf{E} & =\left(\mathbb{H}_{0}-\mathbb{V}-2 \mathcal{M}[\mathbf{E}]-3 \mathcal{N}[\mathbf{E} \otimes \mathbf{E}]\right)^{-1} \mathbb{H}_{0}, \\
\lim _{\mathbf{E}_{\text {in }} \rightarrow 0} \delta_{\mathbf{E}_{\text {in }}} \mathbf{E} & =\left(\mathbb{H}_{0}-\mathbb{V}\right)^{-1} \mathbb{H}_{0}, \\
\delta_{\mathbf{E}_{\text {in }}}^{2} \mathbf{E} & =\left(\delta_{\mathbf{E}_{\text {in }}} \mathbf{E}\right) \mathbb{G}_{0}(2 \mathcal{M}+6 \mathcal{N}[\mathbf{E}])\left(\delta_{\mathbf{E}_{\text {in }}} \mathbf{E}\right)^{2}, \\
\lim _{\mathbf{E}_{\text {in }} \rightarrow 0} \delta_{\mathbf{E}_{\text {in }}}^{2} \mathbf{E} & =2\left(\mathbb{H}_{0}-\mathbb{V}\right)^{-1} \mathcal{M}\left(\mathbb{H}_{0}-\mathbb{V}\right)^{-1} \mathbb{H}_{0}\left(\mathbb{H}_{0}-\mathbb{V}\right)^{-1} \mathbb{H}_{0}, \\
\mathcal{M} & =\frac{1}{2}\left(\mathbb{H}_{0}-\mathbb{V}\right)\left(\lim _{\mathbf{E}_{\text {in }} \rightarrow 0} \delta_{\mathbf{E}_{\text {in }}}^{2} \mathbf{E}\right) \mathbb{G}_{0}\left(\mathbb{H}_{0}-\mathbb{V}\right) \mathbb{G}_{0}\left(\mathbb{H}_{0}-\mathbb{V}\right) .
\end{aligned}
$$

For $\mathcal{N}$, which is the operator form of the third order susceptibility $\chi^{(3)}$, the expression gets even more complicated, but can be obtained through the third derivative, $\mathbb{V}$, and $\mathcal{M}$. For a general discussion see Appendix A.

\subsection{The general stochastic nonlinear Helmholtz equation}

\subsubsection{Nonlinear optics with noise}

As we showed in the previous chapter, the nonlinear Helmholtz equation,

$$
\left(\mathbb{H}_{0}-\mathbb{V}\right) \mathbf{E}-\mathcal{M}[\mathbf{E} \otimes \mathbf{E}]-\mathcal{N}[\mathbf{E} \otimes \mathbf{E} \otimes \mathbf{E}]=0,
$$

can be used to describe well the experiments of nonlinear optics. We will return to this, but to start, we will consider a generally nonlinear Helmholtz equation, by introducing a general polarization operator,

$$
\mathcal{P}[\mathbf{E}]=\mathcal{P}^{(0)}+\mathcal{P}^{(1)} \mathbf{E}+\mathcal{P}^{(2)}[\mathbf{E} \otimes \mathbf{E}]+\mathcal{P}^{(3)}[\mathbf{E} \otimes \mathbf{E} \otimes \mathbf{E}]+\ldots,
$$

and the nonlinear Helmholtz operator,

$$
\mathcal{H}[\mathbf{E}]=\mathbb{H}_{0} \mathbf{E}-\mathcal{P}[\mathbf{E}] .
$$

There is a clear correspondence with the previous notation,

$$
\begin{aligned}
& \mathcal{P}^{(1)}=\mathbb{V}, \\
& \mathcal{P}^{(2)}=\mathcal{M}, \\
& \mathcal{P}^{(3)}=\mathcal{N} .
\end{aligned}
$$

The zeroth component, $\mathcal{P}^{(0)}$, could include any possible free current densities. We will assume $\mathcal{P}^{(0)}=0$ and include any free currents explicitly as sources of the probing (or 


\section{Combining electromagnetic fluctuations and nonlinear optics}

external) field. It makes no difference physically.

As in the linear case, without noise the nonlinear Helmholtz equation is in violation of the FDT [Eq. (2.36)]. Without noise (and free charges) the system would relax to the trivial solution $\mathbf{E}=0$, even though the linear response is nonzero. Therefore, as in the linear case, we need to add a source of noise, the so-called Rytov currents $\mathbf{F}$.

In addition to noise, we add the deterministic probing source $\mathbb{H}_{0} \mathbf{E}_{\text {in }}$ into Eq. (2.71) (as in Section 2.1.2) in order to measure the response of the system, giving finally the stochastic nonlinear Helmholtz equation,

$$
\mathcal{H}[\mathbf{E}]=\mathbf{F}+\mathbb{H}_{0} \mathbf{E}_{\text {in }} .
$$

Notice that without noise or any objects $(\mathcal{P}=0)$, the solution would be $\mathbf{E}=\mathbf{E}_{\text {in }}$. So the source term, placed far away, describes incoming radiation. Alternatively, one can picture this as creating a field $\mathbf{E}_{\text {in }}$ in vacuum and then placing objects described by the linear $(\mathbb{V})$ and nonlinear (e.g. $\mathcal{N}$ ) potentials into this field.

\subsubsection{The nonlinear response operator}

Eq. (2.82) describes a model, which is only meaningful, if it can be connected to physical measurements. The most straightforward experiment that can be performed on an optical system is a response measurement, where the average field is measured as a function of (technically the source of) the incoming field,

$$
\mathcal{R}\left[\mathbb{H}_{0} \mathbf{E}_{\text {in }}\right]=\langle\mathbf{E}\rangle .
$$

The functional derivatives of the response operator $\mathcal{R}$ describe the system experimentally and are assumed to be known. As before, we can represent it as a series,

$$
\mathcal{R}[\mathbf{a}]=\mathcal{R}^{(0)}+\mathcal{R}^{(1)} \mathbf{a}+\mathcal{R}^{(2)}[\mathbf{a} \otimes \mathbf{a}]+\mathcal{R}^{(3)}[\mathbf{a} \otimes \mathbf{a} \otimes \mathbf{a}]+\ldots
$$

Note that the coefficients (operators) are obtained from the $\mathbf{E}_{\text {in }} \rightarrow 0$ limit (near equilibrium),

$$
\mathcal{R}^{(n)}=\frac{1}{n} \lim _{\mathbf{E}_{\text {in }} \rightarrow 0} \frac{\delta^{n}\langle\mathbf{E}\rangle}{\delta \mathbf{E}_{\text {in }}^{n}} .
$$

For example, the equilibrium field is given by $\langle\mathbf{E}\rangle^{\mathrm{eq}}=\mathcal{R}^{(0)}$ and the equilibrium linear response [recall as the quantity entering the FDT in Eq. (2.36)] by

$$
\mathcal{R}^{\prime}[0]=\mathcal{R}^{(1)}=\left(\frac{\delta\langle\mathbf{E}\rangle}{\delta \mathbf{E}_{\text {in }}}\right)^{\text {eq }} \mathbb{G}_{0} .
$$


In order to have a self-consistent theory, we need a way to map $\mathcal{R}$ and $\mathcal{P}$ onto each other. We can extract implicitly the average field $\langle\mathbf{E}\rangle$ from Eqs. (2.82) and (2.78) by applying the free Green's function and reorganizing. This gives us a "scattering picture" of the response measurement,

$$
\mathcal{R}\left[\mathbb{H}_{0} \mathbf{E}_{\text {in }}\right]=\langle\mathbf{E}\rangle=\mathbf{E}_{\text {in }}+\mathbb{G}_{0}\langle\mathbf{F}\rangle+\mathbb{G}_{0}\langle\mathcal{P}[\mathbf{E}]\rangle .
$$

There are two important aspects of this equation:

1. If there are nonlinear objects in the system, the last term, which can be thought of as "normal" scattering, depends on (different order) correlations of the field,

$$
\langle\mathcal{P}[\mathbf{E}]\rangle=\mathcal{P}^{(0)}+\mathcal{P}^{(1)}\langle\mathbf{E}\rangle+\mathcal{P}^{(2)}[\langle\mathbf{E} \otimes \mathbf{E}\rangle]+\mathcal{P}^{(3)}[\langle\mathbf{E} \otimes \mathbf{E} \otimes \mathbf{E}\rangle]+\ldots
$$

Since the correlations depend on the noise, the scattered field also depends on noise in the system. Additional information (the FDT) is required to determine these and we will see below how this can be taken into account with an "effective" or renormalized polarizability, albeit in a perturbative manner.

2. Since we haven't fixed $\mathbf{F}$, it is not really obvious which part (if any) of the scattered field should be attributed to the response of Rytov currents. In fact, we will see in Chapter 5 that this question cannot be answered with equilibrium measurements and FDT alone.

For this and the next chapter, we will assume $\langle\mathbf{F}\rangle=0$ and concentrate on the effects of correlations on the response function through the $\mathbb{G}_{0}\langle\mathcal{P}[\mathbf{E}]\rangle$ term in Eq. (2.87). This was the approach we took in Refs. $[85,86]$ mostly because of practical reasons - it does not contradict with anything as far as we know, it is the "simplest" choice7, and is also assumed in the linear case allowing for easier comparison. Sections 5.2 and 5.3 explore some alternatives and their effects.

Since the response of a nonlinear system depends on the correlations of the field (unlike in the linear case), it can be seen that applying the FDT will not be trivial. This is because the FDT, Eq (2.36), is no longer an explicit but rather an implicit equation for determining the correlations.

\subsubsection{The effective nonlinear Helmholtz equation}

Before turning to the noise and correlations, there is one more missing but very useful piece. This is the deterministic pair of Eq. (2.82), which describes not the propagation

\footnotetext{
${ }^{7}$ Simplicity as a criterium comes up often in field theoretical approaches [3], because one can often make a theory more and more complex (with the added degrees of freedom), but not more and more simple without reaching contradictions. This can, however, be subjective and depend on the particular derivation.
} 


\section{Combining electromagnetic fluctuations and nonlinear optics}

of the full field, but its average instead. We will call it the effective nonlinear Helmholtz equation,

$$
\mathbb{H}_{0}\langle\mathbf{E}\rangle-\tilde{\mathcal{P}}[\langle\mathbf{E}\rangle]=\mathbb{H}_{0} \mathbf{E}_{\text {in }} .
$$

This is essentially the noiseless (bare) Helmholtz equation, except that the effective polarization operator $\tilde{\mathcal{P}}$ is connected directly to the inverse of the response operator,

$$
\tilde{\mathcal{P}}[\langle\mathbf{E}\rangle]=\mathbb{H}_{0}\langle\mathbf{E}\rangle-\mathcal{R}^{-1}[\langle\mathbf{E}\rangle] .
$$

It should be noted that the existence of Eq. (2.89) and $\tilde{\mathcal{P}}$ is essentially a postulation of the fact that the inverse of the response operator $\mathcal{R}$ exists. In the linear case, this is generally a safe assumption (the inverse can be given as a series), but it is not as trivial in the nonlinear case. This is, however, a very useful approximation and we will motivate it below with an explicit calculation.

We assume here that the zeroth order of $\tilde{\mathcal{P}}$ is zero, meaning $\langle\mathbf{E}\rangle^{\text {eq }}=0$ and $\tilde{\mathcal{P}}[0]=0 .{ }^{8}$ To calculate the first few components of the effective polarization operator, we can take successive functional derivatives of Eq. (2.89). For the first one, we take the functional derivative $\delta_{\mathbf{E}_{\text {in }}}$ of both sides (note the chain rule), giving us

$$
\mathbb{H}_{0} \delta_{\mathbf{E}_{\text {in }}}\langle\mathbf{E}\rangle-\tilde{\mathcal{P}}^{\prime}[\langle\mathbf{E}\rangle] \delta_{\mathbf{E}_{\text {in }}}\langle\mathbf{E}\rangle=\mathbb{H}_{0} .
$$

From here we can express either the first derivative of the effective polarization or the linear response,

$$
\begin{aligned}
\tilde{\mathcal{P}}^{\prime}[\langle\mathbf{E}\rangle] & =\mathbb{H}_{0}\left[\mathbb{I}-\left(\delta_{\mathbf{E}_{\text {in }}}\langle\mathbf{E}\rangle\right)^{-1}\right] \\
\delta_{\mathbf{E}_{\text {in }}}\langle\mathbf{E}\rangle & =\left(\mathbb{I}-\mathbb{G}_{0} \tilde{\mathcal{P}}^{\prime}[\langle\mathbf{E}\rangle]\right)^{-1} .
\end{aligned}
$$

Notice that inserting either one into the other will yield a trivial identity. From the first equation we get the first coefficient, the effective linear polarizability or the effective potential $\tilde{\mathbb{V}}$,

$$
\tilde{\mathbb{V}}=\tilde{\mathcal{P}}^{\prime}[0]=\mathbb{H}_{0}\left[\mathbb{I}-\left(\delta_{\mathbf{E}_{\text {in }}}\langle\mathbf{E}\rangle\right)^{-1}\right]^{\text {eq }} .
$$

We can see that this exists if and only if the equilibrium linear response $\lim _{\mathbf{E}_{\text {in }} \rightarrow 0}\left(\delta_{\mathbf{E}_{\text {in }}}\langle\mathbf{E}\rangle\right)^{-1}$ is invertible. This is true for any well-behaving system.

By applying $\delta_{\mathbf{E}_{\text {in }}}$ to Eq. (2.92), we can generate the next order term as before,

$$
\tilde{\mathcal{P}}^{\prime \prime}[\langle\mathbf{E}\rangle]=-\mathbb{H}_{0}\left(\delta_{\mathbf{E}_{\text {in }}}\langle\mathbf{E}\rangle\right)^{-2} \delta_{\mathbf{E}_{\text {in }}}^{2}\langle\mathbf{E}\rangle\left(\delta_{\mathbf{E}_{\text {in }}}\langle\mathbf{E}\rangle\right)^{-1},
$$

\footnotetext{
${ }^{8}$ The extension to a nonzero equilibrium field is trivial, however. One would need to expand around this field instead of zero. For the sake of simplicity, we use $\mathbf{E}_{\text {in }} \rightarrow 0 \Longleftrightarrow\langle\mathbf{E}\rangle \rightarrow 0$.
} 
with the effective second order potential given as

$$
\tilde{\mathcal{M}}=\tilde{\mathcal{P}}^{\prime \prime}[0]=-\mathbb{H}_{0}\left[\left(\delta_{\mathbf{E}_{\text {in }}}\langle\mathbf{E}\rangle\right)^{-2} \delta_{\mathbf{E}_{\text {in }}}^{2}\langle\mathbf{E}\rangle\left(\delta_{\mathbf{E}_{\text {in }}}\langle\mathbf{E}\rangle\right)^{-1}\right]^{\text {eq }} .
$$

As can be seen, this is getting complicated quickly. It is important to note, however, that as in the case of $\tilde{\mathbb{V}}, \tilde{\mathcal{M}}$ exists if and only if the equilibrium linear response $\lim _{\mathbf{E}_{\text {in }} \rightarrow 0}\left(\delta_{\mathbf{E}_{\text {in }}}\langle\mathbf{E}\rangle\right)^{-1}$ is invertible.

Even though the calculation of higher order terms of $\tilde{\mathcal{P}}$ gets impractical, the pattern stays the same and we can make a general statement. If the equilibrium linear response is invertible, there exists an effective nonlinear Helmholtz equation for the average electric field in the form of Eq. (2.89), where the effective polarizability $\tilde{\mathcal{P}}$ is determined by the response operator $\mathcal{R}$.

With the availability of the effective polarization operator, it is also possible to give an alternative form to Eq. (2.87), which defines a connection between the scattering experiment (response operator $\mathcal{R}$ ) and material properties (polarization operator $\mathcal{P}$ ). Subtracting the effective nonlinear Helmholtz equation [Eq. (2.89)] from the average of the stochastic nonlinear Helmholtz equation [Eq. (2.82)], we obtain the following:

$$
\tilde{\mathcal{P}}[\langle\mathbf{E}\rangle]=\langle\mathcal{P}[\mathbf{E}]\rangle+\langle\mathbf{F}\rangle \text {. }
$$

Note that using this in Eq. (2.87) yields again a trivial identity, they are essentially the same equation.

\subsubsection{Fluctuations and noise}

We have seen that when connecting the material properties to response measurements directly [Eq. (2.87)] or through the effective polarization operator [Eq. (2.97)], we encounter the average of the noise $\langle\mathbf{F}\rangle$ and the average polarization $\langle\mathcal{P}[\mathbf{E}]\rangle$. At the end of Section 2.3.2 we argued that $\langle\mathbf{F}\rangle=0$ is a sensible choice, but that still leaves us with the correlations.

Physically, noise is the source of fluctuations, but not the other way around. In equilibrium, however, it can be easier to determine the fluctuations (e.g. through the FDT) and then find the corresponding noise. In the next two chapters we will see that it will make more sense to make assumptions about the noise and determine the generated fluctuations. 


\section{Relation between noise and fluctuations}

If we subtract from the field $\mathbf{E}$ its average $\langle\mathbf{E}\rangle$, we obtain the fluctuations. Even though the terms "noise" and "fluctuations" are sometimes used interchangeably in literature, we will stick with the strict terms here: noise refers to the stochastic source term in a Helmholtz equation and fluctuations are defined by

$$
\delta \mathbf{E}=\mathbf{E}-\langle\mathbf{E}\rangle
$$

Notice that fluctuations have a strictly zero mean,

$$
\langle\delta \mathbf{E}\rangle=0,
$$

whereas the same cannot be necessarily said about the noise $\mathbf{F}$. Nevertheless, both the fluctuations and noise have in general nonzero correlations.

Using Eqs. (2.82) and (2.83), we can show explicitly the "dual" nature of the noise and fluctuations,

$$
\begin{aligned}
\delta \mathbf{E} & =\mathcal{H}^{-1}\left[\mathbb{H}_{0} \mathbf{E}_{\mathrm{in}}+\mathbf{F}\right]-\mathcal{R}\left[\mathbb{H}_{0} \mathbf{E}_{\mathrm{in}}\right], \\
\mathbf{F} & =\mathcal{H}[\langle\mathbf{E}\rangle+\delta \mathbf{E}]-\mathcal{R}^{-1}[\langle\mathbf{E}\rangle] .
\end{aligned}
$$

If we know one, we can in principle calculate the other. The only difficulty lies in inverting the Helmholtz and response operators $\mathcal{H}$ and $\mathcal{R}$.

Alternatively, using the effective nonlinear Helmholtz equation [Eq. (2.89)], we can write down a wave equation for the fluctuations,

$$
\begin{aligned}
\mathbb{H}_{0} \delta \mathbf{E}-\mathcal{Q}_{\langle\mathbf{E}\rangle}[\delta \mathbf{E}] & =\mathbf{F}, \\
\mathcal{Q}_{\langle\mathbf{E}\rangle}[\delta \mathbf{E}] & =\mathcal{P}[\langle\mathbf{E}\rangle+\delta \mathbf{E}]-\tilde{\mathcal{P}}[\langle\mathbf{E}\rangle]
\end{aligned}
$$

We include a subscript in the $\mathcal{Q}$ operator to signify that the dynamics of $\delta \mathbf{E}$ depend (explicitly) on $\langle\mathbf{E}\rangle$.

Notice that, for a linear system $\left(\mathcal{P}_{\text {lin }}=\tilde{\mathcal{P}}_{\text {lin }}=\mathcal{Q}_{\text {lin }}\right)$, Eq. (2.102) reduces to the Helmholtz equation, meaning the fluctuations and the average field are independent. This is not surprising, because due to the superposition principle, we can always separate the field and sources into corresponding sums. This is not true in the nonlinear case. Eq. (2.102) shows clearly that the dynamics of the fluctuations depend on the average field, and Eq. (2.89) shows the opposite case. ${ }^{9}$

${ }^{9}$ It is not a trivial coupling, however. The dynamics of $\langle\mathbf{E}\rangle$ do not depend directly on $\delta \mathbf{E}$ (otherwise it would also be fluctuating). Rather, the average depends on the distribution (that is, the correlations 


\section{Fluctuations and their correlations}

The correlations of the electric field can be written as

$$
\langle\mathbf{E} \otimes \mathbf{E}\rangle=\langle\delta \mathbf{E} \otimes \delta \mathbf{E}\rangle+\langle\mathbf{E}\rangle \otimes\langle\mathbf{E}\rangle
$$

The deterministic part, $\langle\mathbf{E}\rangle \otimes\langle\mathbf{E}\rangle$, is not particularly interesting for us, because a) we assume the full response and therefore this tensor product is known, and b) without free charges or currents this contribution disappears in equilibrium,

$$
\langle\mathbf{E} \otimes \mathbf{E}\rangle^{\mathrm{eq}}=\langle\delta \mathbf{E} \otimes \delta \mathbf{E}\rangle^{\mathrm{eq}} \text {. }
$$

This can be obtained relatively easily from the FDT using the equilibrium linear response [Eq. (2.36)], but what about higher moments that appear in Eqs. (2.97) and $(2.87)$ ?

For linear systems, one can assume that the fluctuations are Gaussian. This is because the electromagnetic action is quadratic, leading necessarily to Gaussian fluctuations. In that case, one can use the Isserlis' theorem to represent higher order moments in terms of two-point correlators. The theorem is more general, but for four (zero-mean) Gaussian variables ${ }^{10}$ we have

$$
\langle A B C D\rangle_{\text {Gaussian }}=\langle A B\rangle\langle C D\rangle+\langle A C\rangle\langle B D\rangle+\langle A D\rangle\langle B C\rangle,
$$

while all products with odd number of terms are zero. For any stochastic variable, knowing all its moments is equivalent to knowing its distribution. This means, for Gaussian fluctuations, the second moment contains all the information. The FDT is therefore enough to fully characterize the fluctuations in equilibrium.

In the nonlinear case, the fluctuations are generally not Gaussian, however, because the distribution function (action) is not quadratic anymore. This has interesting consequences. Since Isserlis' theorem no longer applies, there is no straightforward connection between lower and higher order correlations. Therefore, the FDT in the form of Eq. (2.36) is not enough to fully characterize the fluctuations, even in equilibrium. The higher moments would then remain as free parameters representing either microscopic detail or our lack of knowledge regarding a higher order FDT. This case is discussed in more detail in Chapter 5.

$\left.\left\langle\delta \mathbf{E}^{n}\right\rangle\right)$ of $\mathbf{E}$. Both of them, however, are set by three things: temperature, material properties, and the external field $\mathbf{E}_{\text {in. }}$.

${ }^{10}$ In general for an average of a product of $2 n$ terms, there will be $\frac{(2 n) !}{2^{n} n !}$ terms in the sum, while odd products will be zero. In our case the moments will all appear under the $\mathcal{P}^{(n)}$ operators [Eq. (2.77)], which are symmetric, because the individual components $\mathcal{P}^{(n)}$ are defined as components of a series (or derivatives). This means that all the terms will be identical and instead a factor $\frac{(2 n) !}{2^{n} n !}$ appears. 


\section{Combining electromagnetic fluctuations and nonlinear optics}

In general, we can say that the Helmholtz equation describes the dynamics of the system, but not the distribution of fluctuations. The latter must be obtained by other means, such as the FDT. In the nonlinear case we face two problems: the FDT is in general not sufficient to determine the fluctuations, and the fluctuations become coupled with dynamics through Eq. (2.97). To our advantage, however, it will turn out that these "beyond-FDT" contributions do not contribute in a perturbative model, because the higher order moments only appear in nonlinear terms.

\subsubsection{Summary}

In this section we derived important generic equations that act as a basis for this chapter and the following. Most importantly, we have the three nonlinear Helmholtz equations given by Eqs. (2.82), (2.89), and (2.102),

$$
\begin{aligned}
\mathbb{H}_{0} \mathbf{E}-\mathcal{P}[\mathbf{E}] & =\mathbb{H}_{0} \mathbf{E}_{\mathrm{in}}+\mathbf{F}, \\
\mathbb{H}_{0}\langle\mathbf{E}\rangle-\tilde{\mathcal{P}}[\langle\mathbf{E}\rangle] & =\mathbb{H}_{0} \mathbf{E}_{\mathrm{in}}, \\
\mathbb{H}_{0} \delta \mathbf{E}-\mathcal{Q}_{\langle\mathbf{E}\rangle}[\delta \mathbf{E}] & =\mathbf{F} .
\end{aligned}
$$

Notice that in the current formulation, the nonlinear polarization $\mathcal{P}$ describes the response of the underlying physical material and does not change. By contrast, the effective polarization $\tilde{\mathcal{P}}$ depends explicitly, and $\mathcal{Q}$ implicitly, on the different order correlations of the noise $\mathbf{F}$, which is unknown. These were given in Eqs. (2.97) and (2.103),

$$
\begin{aligned}
\tilde{\mathcal{P}}[\langle\mathbf{E}\rangle] & =\langle\mathcal{P}[\mathbf{E}]\rangle+\langle\mathbf{F}\rangle, \\
\mathcal{Q}_{\langle\mathbf{E}\rangle}[\delta \mathbf{E}] & =\mathcal{P}[\langle\mathbf{E}\rangle+\delta \mathbf{E}]-\tilde{\mathcal{P}}[\langle\mathbf{E}\rangle] .
\end{aligned}
$$

The crucial point is that the effective polarization or potential can additionally be measured experimentally, as in Eq. (2.90),

$$
\tilde{\mathcal{P}}[\langle\mathbf{E}\rangle]=\mathbb{H}_{0}\langle\mathbf{E}\rangle-\mathcal{R}^{-1}[\langle\mathbf{E}\rangle] .
$$

This reduces the degrees of freedom in the theory, but not completely. ${ }^{11}$

The statistical properties of the noise $\mathbf{F}$ (meaning all the moments) remain unknown, mostly because there exists no analogue of the Isserlis' theorem for non-Gaussian variables nor even an FDT for higher order moments. Therefore we also miss the moments of $\mathbf{E}$, specifically the second moment $\left\langle\mathbf{E} \otimes \mathbf{E}^{*}\right\rangle$, which determines the Casimir effect (or general forces in the system) and energy transfer. These are both of great practical importance and interest. To sidestep these issues, we will continue by treating the

\footnotetext{
${ }^{11}$ It creates, in effect, a condition between the average noise $\langle\mathbf{F}\rangle$ and its correlations. See Chapter 5.
} 
nonlinearities perturbatively, starting from equilibrium, for which the regular FDT together with some additional assumptions for the noise $\mathbf{F}$ will be sufficient for practical predictions.

\subsection{Perturbative treatment}

We saw in Sec. 2.3, that for materials with an arbitrary response, $\mathcal{P}[\mathbf{E}]$, we reach a seeming dead end, since it is not clear how to determine higher order moments of the noise. The FDT gives only the second moment of the field in equilibrium in terms of the linear response of the first moment. It is therefore pertinent to turn towards approximations that could give us a leading order departure from the linear theory.

Conveniently, the materials encountered in optics are by far very weakly nonlinear. The linear response of the material almost always dominates over any nonlinear effects. For example, resonator cavities need to be used in order to achieve reasonably efficient frequency mixing (including second/third harmonic generation). On the other hand, all materials are inherently nonlinear. Even vacuum becomes a nonlinear medium if the field strength (energy density) is high enough. In practical cases, the nonlinear effects are barely noticeable, which is why linear optics works so well in the first place.

The idea is to utilize this smallness of the nonlinear response to calculate first order effects. This is of course a double-edged sword, since the deviations from the linear theory are bound to be small, if fields (or temperature for noise strength) are kept small enough such that the approximation is still valid.

\subsubsection{Approximating the nonlinear Helmholtz equation}

The first step is to limit the series of the functional $\mathcal{P}[\mathbf{E}]$. As above, we assume it can be written in a formal series

$$
\mathcal{P}[\mathbf{E}]=\sum_{n=1}^{\infty} \mathcal{P}^{(n)}[\underbrace{\mathbf{E} \otimes \ldots \otimes \mathbf{E}}_{n \text { terms }}] .
$$

Since $\mathbf{E}$ has units, we divide the fields by some characteristic field strength $E_{0}$, giving us

$$
\mathcal{P}[\mathbf{E}]=\sum_{n=1}^{\infty} \mathcal{P}^{(n)} E_{0}^{n}[\underbrace{\frac{\mathbf{E}}{E_{0}} \otimes \ldots \otimes \frac{\mathbf{E}}{E_{0}}}_{n \text { terms }}] .
$$




\section{Combining electromagnetic fluctuations and nonlinear optics}

Assuming the factor in the brackets is of the order unity or smaller, we would need $\mathcal{P}^{(n)} E_{0}^{n}$ to decrease rapidly with increasing $n$. And this is indeed the case, as we know from practice and theory (see Ref. [13]).

As an example, consider one of the most highly nonlinear metamaterial, glass infused with gold or silver nanospheres. Near the plasmonic resonance of the spheres, the electric field is concentrated to very high intensities, which then probes nonlinear interactions in glass. The order of magnitude for the third order susceptibility (second order is negligible due to symmetries) is about $\left|\chi^{(3)}\right| \sim 10^{-16} \frac{\mathrm{m}^{2}}{\mathrm{~V}^{2}}$, while the linear susceptibility is of the order of unity. This means the series becomes divergent roughly at fields reaching $|\mathbf{E}| \sim 10^{8} \frac{\mathrm{V}}{\mathrm{m}}$, which is only slightly below the breakdown voltage of air at $3 \times 10^{6} \frac{\mathrm{V}}{\mathrm{m}}$. On the other hand, in glass we have $\left|\chi^{(3)}\right| \sim 10^{-22} \frac{\mathrm{m}^{2}}{\mathrm{~V}^{2}}$, which corresponds to fields as high as $|\mathbf{E}| \sim 10^{11} \frac{\mathrm{V}}{\mathrm{m}}$. This is nearing the atomic electric field strength (see Ref. [13]), which would cause ionization and therefore conduction. We will assume that the field strengths are well below that.

In conclusion, for practical situations, the series for the polarization field converges very quickly and it is enough to consider only the first few terms. Being mindful that the second order susceptibility is zero for a very large group of materials due to symmetries, we will consider up to third order susceptibility of the material,

$$
\mathcal{P}[\mathbf{E}] \approx \mathbb{V} \mathbf{E}+\mathcal{M}[\mathbf{E} \otimes \mathbf{E}]+\mathcal{N}[\mathbf{E} \otimes \mathbf{E} \otimes \mathbf{E}] .
$$

Furthermore, in the calculations below we will encounter terms which contain products of these quantities. In those cases we will only be keeping the leading order terms. In effect this corresponds to treating $E_{0}$ appearing in Eq. (2.114) as a pseudo-small parameter (since it has units). By neglecting all terms containing $\mathcal{P}^{(2)} \mathcal{P}^{(2)}, \mathcal{P}^{(2)} \mathcal{P}^{(3)}$, $\mathcal{P}^{(3)} \mathcal{P}^{(3)}$, or any higher combination, all results will be approximate to the order of $E_{0}^{3}$. In fact, keeping such terms would be misleading since then one would need to include fourth and higher order susceptibilities, because their contributions would be of similar significance.

In summary, we approximate the polarization operator by Eq. (2.115) and obtain the truncated form of the stochastic nonlinear Helmholtz equation (2.82),

$$
\left(\mathbb{H}_{0}-\mathbb{V}\right) \mathbf{E}-\mathcal{M}[\mathbf{E} \otimes \mathbf{E}]-\mathcal{N}[\mathbf{E} \otimes \mathbf{E} \otimes \mathbf{E}]=\mathbb{H}_{0} \mathbf{E}_{\mathrm{in}}+\mathbf{F} .
$$

We also disregard or discard all terms which have more than one factor of either $\mathcal{M}$ or $\mathcal{N}$. 


\subsubsection{The effective nonlinear Helmholtz equation}

Eq. (2.116) gives the equation for the stochastic electric field. In order to use the FDT, however, we need to know how the average field $\overline{\mathbf{E}}=\langle\mathbf{E}\rangle$ responds to the incoming field. This is given by the effective nonlinear Helmholtz equation [Eq. (2.89)],

$$
\mathbb{H}_{0} \overline{\mathbf{E}}-\tilde{\mathcal{P}}[\overline{\mathbf{E}}]=\mathbb{H}_{0} \mathbf{E}_{\text {in }}
$$

The effective polarization is now given by Eq. (2.97). Including the truncated $\mathcal{P}[\mathbf{E}]$ of Eq. (2.115) and $\mathbf{E}=\overline{\mathbf{E}}+\delta \mathbf{E}$, we get

$$
\begin{aligned}
\tilde{\mathcal{P}}[\overline{\mathbf{E}}] & =\langle\mathcal{P}[\mathbf{E}]\rangle+\langle\mathbf{F}\rangle \\
& =\mathbb{V}\langle\mathbf{E}\rangle+\mathcal{M}[\langle\mathbf{E} \otimes \mathbf{E}\rangle]+\mathcal{N}[\langle\mathbf{E} \otimes \mathbf{E} \otimes \mathbf{E}\rangle]+\langle\mathbf{F}\rangle \\
& =\mathbb{V} \overline{\mathbf{E}}+\langle\mathbf{F}\rangle \\
& +\mathcal{M}[\overline{\mathbf{E}} \otimes \overline{\mathbf{E}}]+\mathcal{M}[\langle\delta \mathbf{E} \otimes \delta \mathbf{E}\rangle] \\
& +\mathcal{N}[\overline{\mathbf{E}} \otimes \overline{\mathbf{E}} \otimes \overline{\mathbf{E}}]+3 \mathcal{N}[\langle\delta \mathbf{E} \otimes \delta \mathbf{E}\rangle \otimes \overline{\mathbf{E}}]+\mathcal{N}[\langle\delta \mathbf{E} \otimes \delta \mathbf{E} \otimes \delta \mathbf{E}\rangle] .
\end{aligned}
$$

In the last line we used the symmetric properties of $\mathcal{N}$ (see Section 2.2.1), whereby

$$
\mathcal{N}[\langle\delta \mathbf{E} \otimes \delta \mathbf{E}\rangle \otimes \overline{\mathbf{E}}]=\mathcal{N}[\overline{\mathbf{E}} \otimes\langle\delta \mathbf{E} \otimes \delta \mathbf{E}\rangle]=\mathcal{N}[\langle\delta \mathbf{E} \otimes \overline{\mathbf{E}} \otimes \delta \mathbf{E}\rangle] .
$$

Next we use again the approximation that we can neglect terms that are "doubly nonlinear". This means that, to leading order in $\chi^{(2)}$ and $\chi^{(3)}$, it is possible to use the correlator of the linear fluctuations $\langle\delta \mathbf{E} \otimes \delta \mathbf{E}\rangle$ under the nonlinear operators $\mathcal{M}$ and $\mathcal{N}$. Crucially, they are independent of the average field $\overline{\mathbf{E}}$, as shown in Section 2.1.3. Linear fluctuations are also Gaussian, so the third moment vanishes, $\langle\delta \mathbf{E} \otimes \delta \mathbf{E} \otimes \delta \mathbf{E}\rangle=0$. This means we can collect the orders of $\overline{\mathbf{E}}$ in the effective nonlinear Helmholtz equation, with the exception of $\langle\mathbf{F}\rangle$,

$$
\begin{gathered}
\left(\mathbb{H}_{0}-\mathbb{V}-3 \mathcal{N}[\langle\delta \mathbf{E} \otimes \delta \mathbf{E}\rangle]\right) \overline{\mathbf{E}}-\mathcal{M}[\overline{\mathbf{E}} \otimes \overline{\mathbf{E}}]-\mathcal{N}[\overline{\mathbf{E}} \otimes \overline{\mathbf{E}} \otimes \overline{\mathbf{E}}] \\
=\mathbb{H}_{0} \mathbf{E}_{\text {in }}+\langle\mathbf{F}\rangle+\mathcal{M}[\langle\delta \mathbf{E} \otimes \delta \mathbf{E}\rangle] .
\end{gathered}
$$

All terms in this equation depend explicitly on $\overline{\mathbf{E}}$ with the possible exception of $\langle\mathbf{F}\rangle$.

As mentioned above, the $\mathbf{E}$-dependence of $\langle\mathbf{F}\rangle$ can not be determined exactly from our current approach. Nonzero $\langle\mathbf{F}\rangle$ in equilibrium would clash with the "no free charges" assertion, giving a nonzero mean field as $\mathbf{E}_{\text {in }} \rightarrow 0$. Nonzero response to the electric field is more difficult to rule out on physical grounds. However, any possible E-dependence can at least mathematically be absorbed into the bare susceptibilities, leaving us with $\langle\mathbf{F}\rangle=0$. 
In stationary systems the time-domain correlators can only depend on time differences [53]. In Fourier space, therefore, the correlators must be delta-correlated in frequencies (similar to the Fourier transform of the time domain responses as seen in Section 2.2.1),

$$
\left\langle\delta \mathbf{E}_{\omega} \otimes \delta \mathbf{E}_{\omega^{\prime}}\right\rangle=\delta\left(\omega+\omega^{\prime}\right)\langle\delta \mathbf{E} \otimes \delta \mathbf{E}\rangle_{\omega} .
$$

The last source term in Eq. (2.120) then vanishes:

$$
\mathcal{M}[\langle\delta \mathbf{E} \otimes \delta \mathbf{E}\rangle](\mathbf{r}, \omega)_{i}=\delta(\omega) \frac{\omega^{2}}{c^{2}} \int_{-\infty}^{\infty} \mathrm{d} \omega^{\prime} \chi_{i j k}^{(2)}\left(\mathbf{r} ; 0, \omega^{\prime},-\omega^{\prime}\right)\left\langle\delta E_{j}(\mathbf{r}) \delta E_{k}(\mathbf{r})\right\rangle_{\omega^{\prime}}=0
$$

because the integral over $\omega^{\prime}$ is independent of $\omega$, and $\delta(\omega) \frac{\omega^{2}}{c^{2}}=0 .{ }^{12}$

With these considerations, the effective Helmholtz equation can be written in its final form,

$$
\left(\mathbb{H}_{0}-\mathbb{V}-3 \mathcal{N}[\langle\delta \mathbf{E} \otimes \delta \mathbf{E}\rangle]\right) \overline{\mathbf{E}}-\mathcal{M}[\overline{\mathbf{E}} \otimes \overline{\mathbf{E}}]-\mathcal{N}[\overline{\mathbf{E}} \otimes \overline{\mathbf{E}} \otimes \overline{\mathbf{E}}]=\mathbb{H}_{0} \mathbf{E}_{\text {in }}
$$

This is the effective counterpart to Eq. (2.116). The nonlinear coefficients are the same due to retaining only leading order terms in $\chi^{(2)}$ and $\chi^{(3)}$. As opposed to the linear case [see Eqs. (2.44) and (2.45)], the linear terms in effective and bare equations are different. As mentioned in Section 2.3, this signifies the breakdown of the superposition principle and gives meaning to the term "effective" Helmholtz equation - the average field $\overline{\mathbf{E}}$ feels different (effective or renormalized) dielectric properties compared to the stochastic field $\mathbf{E}$.

\subsubsection{The effective potential and dielectric function}

Using Eq. (2.121), the operator $\mathcal{N}[\langle\delta \mathbf{E} \otimes \delta \mathbf{E}\rangle]$ (see Section 2.2.3) in Eq. (2.123) can be written explicitly as

$$
\begin{aligned}
\mathcal{N}[\langle\delta \mathbf{E} \otimes \delta \mathbf{E}\rangle]\left(\mathbf{r}, \mathbf{r}^{\prime}, \omega\right)_{i j} & =\delta\left(\mathbf{r}-\mathbf{r}^{\prime}\right) \frac{\omega^{2}}{c^{2}} \int_{-\infty}^{\infty} \mathrm{d} \omega^{\prime} \chi_{i j k l}^{(3)}\left(\mathbf{r} ;-\omega, \omega, \omega^{\prime},-\omega^{\prime}\right) \\
& \times\left\langle\delta E_{k}(\mathbf{r}) \delta E_{l}(\mathbf{r})\right\rangle_{\omega^{\prime}} .
\end{aligned}
$$

As can be seen, this is no longer an operator over frequencies. Rather, it is exactly the same type of operator as the dielectric potential $\mathbb{V}$. Therefore, we can define the effective

\footnotetext{
${ }^{12}$ In any case the Helmholtz equation is not a very convenient description in the static case.
} 
potential as

$$
\begin{aligned}
\tilde{\mathbb{V}} & =\mathbb{V}+\mathbb{N} \\
\mathbb{N} & =3 \mathcal{N}[\langle\delta \mathbf{E} \otimes \delta \mathbf{E}\rangle] .
\end{aligned}
$$

As in the linear case (see Eq. (2.21)), we can also define a corresponding effective dielectric function

$$
\begin{aligned}
\tilde{\varepsilon}_{i j}(\mathbf{r}, \omega) & =\varepsilon_{i j}(\mathbf{r}, \omega)+N_{i j}(\mathbf{r}, \omega), \\
N_{i j}(\mathbf{r}, \omega) & =3 \int_{-\infty}^{\infty} \mathrm{d} \omega^{\prime} \chi_{i j k l}^{(3)}\left(\mathbf{r} ;-\omega, \omega, \omega^{\prime},-\omega^{\prime}\right)\left\langle\delta \mathbf{E}_{k}(\mathbf{r}) \otimes \delta \mathbf{E}_{l}(\mathbf{r})\right\rangle_{\omega^{\prime}}
\end{aligned}
$$

which is sometimes easier to work with than the full potential operator.

Using the effective dielectric potential (2.125), the effective nonlinear Helmholtz equation can be written as,

$$
\left(\mathbb{H}_{0}-\tilde{\mathbb{V}}\right) \overline{\mathbf{E}}-\mathcal{M}[\overline{\mathbf{E}} \otimes \overline{\mathbf{E}}]-\mathcal{N}[\overline{\mathbf{E}} \otimes \overline{\mathbf{E}} \otimes \overline{\mathbf{E}}]=\mathbb{H}_{0} \mathbf{E}_{\text {in }}
$$

This is the equation that determines the results of optical (including linear and nonlinear scattering) measurements.

\subsubsection{The linear response}

The linear response can be determined directly from Eq. (2.129),

$$
\begin{aligned}
\frac{\delta \overline{\mathbf{E}}}{\delta \mathbf{E}_{\text {in }}} \mathbb{G}_{0} & =\left(\mathbb{H}_{0}-\tilde{\mathbb{V}}-2 \mathcal{M}[\overline{\mathbf{E}}]-3 \mathcal{N}[\overline{\mathbf{E}} \otimes \overline{\mathbf{E}}]\right)^{-1} \\
& =\tilde{\mathbb{G}}+2 \tilde{\mathbb{G}} \mathcal{M}[\overline{\mathbf{E}}] \tilde{\mathbb{G}}+3 \tilde{\mathbb{G}} \mathcal{N}[\overline{\mathbf{E}} \otimes \overline{\mathbf{E}}] \tilde{\mathbb{G}} \\
\tilde{\mathbb{G}} & =\lim _{\mathbf{E}_{\text {in }} \rightarrow 0}\left(\frac{\delta \overline{\mathbf{E}}}{\delta \mathbf{E}_{\text {in }}} \mathbb{G}_{0}\right) \\
& =\left(\mathbb{H}_{0}-\tilde{\mathbb{V}}\right)^{-1} \\
& =\mathbb{G}+3 \mathbb{G} \mathcal{N}[\langle\delta \mathbf{E} \otimes \delta \mathbf{E}\rangle] \mathbb{G} .
\end{aligned}
$$

Notice that we use the Green's function $\mathbb{G}$ instead of the linear response $\tilde{\mathbb{G}}$ in the second term of Eq. (2.131). This holds since one can neglect higher order terms in $\chi^{(3)}$. 


\subsection{Summary}

By introducing fluctuations into the equations of nonlinear optics, we saw that the wave equations for the stochastic field and average field differ more than by an additional source term. Indeed, the whole equation must change, because the superposition principle no longer applies. Thus we obtained the effective and stochastic (or bare) Helmholtz equation describing the dynamics of the average and the fluctuating field, respectively.

In the perturbative case, keeping only the leading order terms of the second and third order susceptibilities and using zero-mean noise, the higher order terms remain the same. The difference between the effective and bare electromagnetic potential, however, depends on the fluctuations themselves,

$$
\tilde{\mathbb{V}}=\mathbb{V}+3 \mathcal{N}[\langle\delta \mathbf{E} \otimes \delta \mathbf{E}\rangle]
$$

This will be the main object of study, together with the fluctuations and noise themselves, in Chapters 3 and 4, where we apply the formalism developed in this chapter to systems in equilibrium and out of thermal equilibrium, respectively.

It is important to stress, however, that these results rely quite heavily on the assumption that in the bare Helmholtz equation [Eq. (2.116)] the noise $\mathbf{F}$ has a zero mean even out of equilibrium. This condition is relaxed and investigated more thoroughly in Chapter 5. 


\section{Fluctuational electrodynamics for nonlinear materials in equilibrium}

In this chapter we study optically nonlinear systems at rest, in the absence of unbound charges or external fields, and with the whole system at the same temperature. While a lot of interesting effects of nonlinear optics only manifest at high field intensities or interaction lengths (such as optical fibers), it is just a fact of nature that most materials are only weakly nonlinear. Nevertheless, this dependence on the magnitude of the external field is what separates linear and nonlinear materials. It might therefore seem odd to look at systems where no external fields are present to push the system into a nonlinear regime. However, we can never completely remove fluctuations, which will be probing both the linear and nonlinear properties of the system, even when the system is at rest (and even at zero temperature due to quantum fluctuations). Furthermore, in this chapter and the next, we will make extensive use of the fact that the nonlinear contribution is small. This allows us to treat the problem perturbatively, keeping only the leading order terms.

We start from the stochastic and effective nonlinear Helmholtz equations (SNHE and ENHE) obtained at the end of Chapter 2 [Eqs. (2.116) and (2.129)] and the effective electromagnetic potential given in Eq. (2.125). These equations are generally valid, if one can supply or determine the correct noise $\mathbf{F}$ or fluctuations $\delta \mathbf{E}$. In this chapter we study the system in equilibrium and can therefore directly use the FDT to determine the fluctuations. We do need to use $\langle\mathbf{F}\rangle=0$, however.

These fluctuations together with the nonlinear response give rise to an effective (noisedependent) electromagnetic potential or dielectric function. We explore both its behavior in Section 3.2 for a static system and also for systems consisting of multiple objects that can move relative to each other. The latter case leads to nonlinear contributions to the so-called combination formula of the linear responses of individual objects.

We finish with a practical example in Section 3.3: the equilibrium Casimir force. 


\subsection{Electromagnetic fluctuations and noise in equilibrium}

\subsubsection{Equilibrium fluctuations}

The equilibrium fluctuations are given directly by the FDT [Eq. (2.36)] and the equilibrium linear response [Eq. (2.131)],

$$
\begin{aligned}
\left\langle\delta \mathbf{E}_{\omega} \otimes \delta \mathbf{E}_{\omega^{\prime}}^{*}\right\rangle^{\mathrm{eq}} & =\delta\left(\omega-\omega^{\prime}\right) \frac{b(\omega)}{2 i}\left(\mathbb{H}_{0}-\tilde{\mathbb{V}}^{\mathrm{eq}}\right)_{\mathrm{AH}}^{-1}, \\
& =\delta\left(\omega-\omega^{\prime}\right) \frac{b(\omega)}{2 i}\left(\mathbb{H}_{0}-\mathbb{V}-3 \mathcal{N}\left[\langle\delta \mathbf{E} \otimes \delta \mathbf{E}\rangle^{\mathrm{eq}}\right]\right)_{\mathrm{AH}}^{-1},
\end{aligned}
$$

where we used zero-mean noise $\langle\mathbf{F}\rangle=0$. The superscript in $\tilde{\mathbb{V}}^{\text {eq }}$ denotes that the expression in Eq. (2.125) is evaluated in equilibrium.

As can be seen, this is an implicit equation for the fluctuations. However, because within a perturbative expansion only leading order terms in $\chi^{(3)}$ need to be kept, this equation can be closed by using linear fluctuations on the right hand side. This will be discussed in more detail in Section 3.2.

\subsubsection{Equilibrium noise - equivalence of Rytov theory and the FDT}

By setting $\mathbf{E}_{\text {in }}=\overline{\mathbf{E}}=0$ in Eq. (2.116), we can directly extract the equilibrium noise, which are also known as Rytov currents,

$$
\mathbf{F}^{\mathrm{eq}}=\left(\mathbb{H}_{0}-\mathbb{V}\right) \delta \mathbf{E}^{\mathrm{eq}}-\mathcal{M}\left[\delta \mathbf{E}^{\mathrm{eq}} \otimes \delta \mathbf{E}^{\mathrm{eq}}\right]-\mathcal{N}\left[\delta \mathbf{E}^{\mathrm{eq}} \otimes \delta \mathbf{E}^{\mathrm{eq}} \otimes \delta \mathbf{E}^{\mathrm{eq}}\right] .
$$

Note that $\left\langle\mathbf{F}^{\mathrm{eq}}\right\rangle=0$ to leading order in $\mathcal{M}$ and $\mathcal{N}$ (see discussion before Eq. (2.129) why $\left.\mathcal{M}\left[\langle\delta \mathbf{E} \otimes \delta \mathbf{E}\rangle^{\mathrm{eq}}\right]=0\right)$.

From the above equation we can determine the equilibrium correlator of the noise by keeping only leading order terms,

$$
\begin{aligned}
\left\langle\mathbf{F}_{\omega} \otimes \mathbf{F}_{\omega^{\prime}}^{*}\right)^{\mathrm{eq}} & =\left(\mathbb{H}_{0}-\mathbb{V}\right)_{\omega}\left\langle\delta \mathbf{E}_{\omega} \otimes \delta \mathbf{E}_{\omega^{\prime}}^{*}\right\rangle^{\mathrm{eq}}\left(\mathbb{H}_{0}-\mathbb{V}\right)_{\omega^{\prime}}^{\dagger} \\
& -\left(\mathbb{H}_{0}-\mathbb{V}\right)_{\omega}\left\langle\delta \mathbf{E}_{\omega} \otimes \mathcal{M}[\delta \mathbf{E} \otimes \delta \mathbf{E}]_{\omega^{\prime}}^{*}\right)^{\mathrm{eq}} \\
& -\left(\mathbb{H}_{0}-\mathbb{V}\right)_{\omega}\left\langle\delta \mathbf{E}_{\omega} \otimes \mathcal{N}[\delta \mathbf{E} \otimes \delta \mathbf{E} \otimes \delta \mathbf{E}]_{\omega^{\prime}}^{*}\right\rangle^{\mathrm{eq}} \\
& -\left\langle\mathcal{M}[\delta \mathbf{E} \otimes \delta \mathbf{E}]_{\omega} \otimes \delta \mathbf{E}_{\omega^{\prime}}^{*}\right\rangle^{\mathrm{eq}}\left(\mathbb{H}_{0}-\mathbb{V}\right)_{\omega^{\prime}}^{\dagger} \\
& -\left\langle\mathcal{N}[\delta \mathbf{E} \otimes \delta \mathbf{E} \otimes \delta \mathbf{E}]_{\omega} \otimes \delta \mathbf{E}_{\omega^{\prime}}^{*}\right\rangle^{\mathrm{eq}}\left(\mathbb{H}_{0}-\mathbb{V}\right)_{\omega^{\prime}}^{\dagger} .
\end{aligned}
$$

Note that we can write $(\mathbb{O} \mathbf{v})^{*}=\mathbf{v}^{*} \mathbb{O}^{\dagger}$. For the terms involving $\mathcal{M}$ and $\mathcal{N}$, we can assume that $\delta \mathbf{E}$ is Gaussian, because any non-Gaussianity would give higher than leading 
order contributions in $\chi^{(2)}$ and $\chi^{(3)}$. The third moment of a zero-mean Gaussian variable is zero, and the fourth moment is given by the Isserlis' theorem, Eq. (2.106). Since the $\mathcal{N}$ operator is symmetric (see Section 2.2), we have

$$
\left\langle\mathcal{N}[\delta \mathbf{E} \otimes \delta \mathbf{E} \otimes \delta \mathbf{E}]_{\omega} \otimes \delta \mathbf{E}_{\omega^{\prime}}^{*}\right\rangle^{\mathrm{eq}}=3 \mathcal{N}\left[\langle\delta \mathbf{E} \otimes \delta \mathbf{E}\rangle^{\mathrm{eq}}\right]_{\omega}\left\langle\delta \mathbf{E}_{\omega} \otimes \delta \mathbf{E}_{\omega^{\prime}}^{*}\right\rangle^{\mathrm{eq}} .
$$

Making use of the equilibrium effective potential from Eq. (2.125), the equilibrium noise correlator can therefore be written as

$$
\left\langle\mathbf{F}_{\omega} \otimes \mathbf{F}_{\omega^{\prime}}^{*}\right\rangle^{\mathrm{eq}}=\left(\mathbb{H}_{0}-\tilde{\mathbb{V}}^{\mathrm{eq}}\right)_{\omega}\left\langle\delta \mathbf{E}_{\omega} \otimes \delta \mathbf{E}_{\omega^{\prime}}^{*}\right\rangle^{\mathrm{eq}}\left(\mathbb{H}_{0}-\tilde{\mathbb{V}}^{\mathrm{eq}}\right)_{\omega^{\prime}}^{\dagger}
$$

Using the equilibrium fluctuations [Eq. (3.1)], this can be further simplified as

$$
\left\langle\mathbf{F}_{\omega} \otimes \mathbf{F}_{\omega^{\prime}}^{*}\right\rangle^{\mathrm{eq}}=-\delta\left(\omega-\omega^{\prime}\right) \frac{b(\omega)}{2 i}\left(\mathbb{H}_{0}-\tilde{\mathbb{V}}^{\mathrm{eq}}\right)_{\mathrm{AH}} .
$$

Eq. (3.6) can be considered the "noise-form" or Rytov form of the FDT [compare with Eq. (3.1)].

\section{Vacuum fluctuations}

Notice that the operator

$$
\mathbb{H}_{0}=\nabla \times \nabla \times-\frac{\omega^{2}}{c^{2}} \mathbb{I},
$$

in Eq. (3.6) is formally real and symmetric, so one might wonder: how can it have an anti-Hermitian (or imaginary) part? It turns out that the imaginary part of the free Green's function $\mathbb{G}_{0}=\mathbb{H}_{0}^{-1}$ is indeed zero except in the following limit,

$$
\lim _{\mathbf{r} \rightarrow \mathbf{r}^{\prime}} \operatorname{Im} \mathbb{G}_{0}\left(\mathbf{r}, \mathbf{r}^{\prime}\right)_{i j}=\frac{1}{6 \pi} \frac{\omega}{c} \delta_{i j}
$$

This is a well known result for the vacuum absorption (also known as the environment dust, see Ref. [29] and the bulk calculation in Section B.3). It is called "dust", because the contribution is infinitesimal compared to regular matter. This can be seen by comparing Eq. (3.8) with the definition of the potential operator in Eq. (2.21) - the potential diverges at equal points whereas the dust is finite. They are, however, both important since vacuum has infinite volume.

To illustrate this, we give Eq. (3.6) explicitly for vacuum $\left(\tilde{\mathbb{V}}=\tilde{\mathbb{V}}^{\text {eq }}=0\right)$ and nonvacuum. Being strict, we let $V_{\text {na }}$ denote the space where no absorption is present (prac- 
tically, this could denote vacuum if the objects have finite size),

$$
\frac{1}{2 i}\left[\tilde{\varepsilon}_{i j}^{\mathrm{eq}}\left(\mathbf{r} \in V_{\mathrm{na}}\right)\right]_{\mathrm{AH}}=0 .
$$

The noise can then be separated as

$$
\begin{aligned}
& \left\langle\mathbf{F}_{\omega}\left(\mathbf{r} \in V_{\mathrm{na}}\right) \otimes \mathbf{F}_{\omega^{\prime}}^{*}\left(\mathbf{r}^{\prime} \in V_{\mathrm{na}}\right)\right\rangle_{i j}^{\mathrm{eq}}= \begin{cases}-\delta\left(\omega-\omega^{\prime}\right) b(\omega) 6 \pi \frac{c}{\omega} \delta_{i j}, & \mathbf{r}=\mathbf{r}^{\prime}, \\
0, & \text { otherwise, }\end{cases} \\
& \left\langle\mathbf{F}_{\omega}\left(\mathbf{r} \notin V_{\mathrm{na}}\right) \otimes \mathbf{F}_{\omega^{\prime}}^{*}\left(\mathbf{r}^{\prime} \notin V_{\mathrm{na}}\right)\right\rangle_{i j}^{\mathrm{eq}}=\delta\left(\omega-\omega^{\prime}\right) \delta\left(\mathbf{r}-\mathbf{r}^{\prime}\right) b(\omega) \frac{1}{2 i}\left[\tilde{\varepsilon}_{i j}^{\mathrm{eq}}(\mathbf{r})\right]_{\mathrm{AH}} .
\end{aligned}
$$

Note that the vacuum dust does not affect the non-vacuum fluctuations, since the deltafunction is divergent at $\mathbf{r}=\mathbf{r}^{\prime}$.

\subsection{The effective dielectric function in equilibrium}

As can be seen from Eqs. (2.129) and (3.1), in most cases, e.g. for optics (response measurements) and the Casimir effect, the influence of nonlinear effects can be taken into account with an effective potential [Eq. (2.125)],

$$
\tilde{\mathbb{V}}^{\mathrm{eq}}=\mathbb{V}+3 \mathcal{N}\left[\langle\delta \mathbf{E} \otimes \delta \mathbf{E}\rangle^{\mathrm{eq}}\right],
$$

the effective linear response [Eq. (2.131)],

$$
\tilde{\mathbb{G}}=\left(\mathbb{H}_{0}-\tilde{\mathbb{V}}^{\mathrm{eq}}\right)^{-1}=\mathbb{G}+3 \mathbb{G} \mathcal{N}\left[\langle\delta \mathbf{E} \otimes \delta \mathbf{E}\rangle^{\mathrm{eq}}\right] \mathbb{G},
$$

(no superscript necessary, because $\tilde{\mathbb{G}}$ is already defined in equilibrium), or the effective dielectric function [Eq. (2.127)],

$$
\begin{aligned}
\tilde{\varepsilon}_{i j}^{\mathrm{eq}}(\mathbf{r}, \omega) & =\varepsilon_{i j}(\mathbf{r}, \omega)+N_{i j}^{\mathrm{eq}}(\mathbf{r}, \omega), \\
N_{i j}^{\mathrm{eq}}(\mathbf{r}, \omega) & =3 \int_{-\infty}^{\infty} \mathrm{d} \omega^{\prime} \chi_{i j k l}^{(3)}\left(\mathbf{r} ;-\omega, \omega, \omega^{\prime},-\omega^{\prime}\right)\left\langle\delta \mathbf{E}_{k}(\mathbf{r}) \otimes \delta \mathbf{E}_{l}(\mathbf{r})\right\rangle_{\omega^{\prime}}^{\mathrm{eq}} .
\end{aligned}
$$

The equilibrium fluctuations are given by Eq. (3.1), but since only leading order terms in $\chi^{(3)}$ are kept, it is sufficient for nonlinear terms to use

$$
\left\langle\delta \mathbf{E}_{\omega} \otimes \delta \mathbf{E}_{\omega^{\prime}}^{*}\right\rangle^{\mathrm{eq}}=\delta\left(\omega-\omega^{\prime}\right) b(\omega) \operatorname{Im} \mathbb{G}(\omega)+\mathcal{O}\left(\chi^{(3)}\right) .
$$

Notice also that the electric field is real-valued, so in Fourier space $\delta \mathbf{E}_{\omega^{\prime}}^{*}=\delta \mathbf{E}_{-\omega^{\prime}}$. Ad- 
ditionally, we used $\mathbb{G}=\mathbb{G}^{\mathrm{T}}$ above. This is true because for linear systems in equilibrium the response must be symmetric, as there are no mechanisms to break microreversibility.

With these considerations, the following expression for the effective dielectric function in equilibrium can be obtained,

$$
N_{i j}^{\mathrm{eq}}(\mathbf{r}, \omega)=3 \int_{-\infty}^{\infty} \mathrm{d} \omega^{\prime} \chi_{i j k l}^{(3)}\left(\mathbf{r} ;-\omega, \omega, \omega^{\prime},-\omega^{\prime}\right) b\left(\omega^{\prime}\right) \operatorname{Im} \mathbb{G}_{k l}\left(\mathbf{r}, \mathbf{r} ; \omega^{\prime}\right) .
$$

The effective potential and linear response can be expressed through this,

$$
\begin{aligned}
\mathbb{V}^{\mathrm{eq}} & =\mathbb{V}+\mathbb{N}^{\mathrm{eq}}, \\
\widetilde{\mathbb{G}} & =\mathbb{G}+\mathbb{G N}^{\mathrm{eq}} \mathbb{G}, \\
\mathbb{N}_{i j}^{\mathrm{eq}}\left(\mathbf{r}, \mathbf{r}^{\prime}, \omega\right) & =\delta\left(\mathbf{r}-\mathbf{r}^{\prime}\right) N_{i j}^{\mathrm{eq}}(\mathbf{r}, \omega) .
\end{aligned}
$$

Therefore it is often sufficient to consider the properties of $N_{i j}^{\mathrm{eq}}(\mathbf{r}, \omega)$.

\section{Integrating over positive frequencies}

Considering Eq. (3.17), it is often more convenient (for example for sign analysis or numerical integration) to integrate over positive frequencies only. This can be easily accomplished by noting three properties of the terms in this expression:

1. $\mathbb{G}=\mathbb{G}^{\mathrm{T}}$ due to micro-reversibility,

2. $\operatorname{Im} \mathbb{G}(\omega)=-\operatorname{Im} \mathbb{G}(-\omega)$, because it represents a real quantity (the correlations of $\delta \mathbf{E})$ in time domain,

3. $\chi_{i j k l}^{(3)}\left(\mathbf{r} ;-\omega, \omega, \omega^{\prime},-\omega^{\prime}\right)=\chi_{i j l k}^{(3)}\left(\mathbf{r} ;-\omega, \omega,-\omega^{\prime}, \omega^{\prime}\right)$ due to the intrinsic symmetry discussed in Section 2.2.

Putting these together, the integral in Eq. (3.17) can be written as

$$
\begin{aligned}
N_{i j}^{\mathrm{eq}}(\mathbf{r}, \omega) & =3 \int_{0}^{\infty} \mathrm{d} \omega^{\prime} \chi_{i j k l}^{(3)}\left(\mathbf{r} ;-\omega, \omega, \omega^{\prime},-\omega^{\prime}\right) a\left(\omega^{\prime}\right) \operatorname{Im} \mathbb{G}_{k l}\left(\mathbf{r}, \mathbf{r} ; \omega^{\prime}\right), \\
a(\omega) & =b(\omega)-b(-\omega) \\
& =\frac{\hbar}{\pi \varepsilon_{0}} \frac{\omega^{2}}{c^{2}} \operatorname{coth}\left(\frac{\hbar \omega}{2 k_{\mathrm{B}} T}\right) .
\end{aligned}
$$

$a(\omega)$ is now the familiar coefficient from Refs. $[52,51]$. Both forms are used in the thesis. Mostly the negative frequencies are kept, but for final force and heat radiation 


\section{Fluctuational electrodynamics for nonlinear materials in equilibrium}

formulas it is useful to consider positive frequencies for sign analysis.

Divergence of $\operatorname{Im} \mathbb{G}_{k l}\left(\mathbf{r}, \mathbf{r} ; \omega^{\prime}\right)$

One can see in Eq. (3.17) the appearance of the local part of $\operatorname{Im} \mathbb{G}$. It is well known that the imaginary part of the Green's function $\mathbb{G}$ diverges as $\mathbf{r}^{\prime} \rightarrow \mathbf{r}$ for absorbing media [38] (see also the bulk calculation in Appendix B); this problem arises generally in field theories [40]. For example, Ref. [62] makes use of a rigid sphere approximation for the delta function (thus "smearing out" the singularity), which ultimately has the same effect as introducing an ultraviolet cut-off to classical field theories. In essence, this reflects the breakdown of the field theory at distances much smaller than the coarse graining length.

There are, however, some ways around this issue that do not necessitate a direct evaluation of this singularity. The most simple is to just consider non-absorbing materials. This is of course a very significant simplification and we will only use it to evaluate $N_{i j}^{\mathrm{eq}}(\mathbf{r}, \omega)$ directly for a planar surface below.

More commonly, the absolute numerical values of $N^{\text {eq }}$ are not interesting by themselves. Instead, often differences $N_{\alpha}-N_{\beta}$ can be considered. For example in order to calculate the Casimir force (see Section 3.3), the relevant quantity is $N\left(d=d_{1}\right)-$ $N\left(d=d_{2}\right)$, where $d$ is the distance between plates. In the next chapter, on the other hand, $N(\Delta T=0)-N(\Delta T)$ is interesting, where $\Delta T$ is the temperature difference between two objects. In both of these cases, it turns out that the singular part of $N$ does not change with those variables, so they cancel out.

\subsection{1. $\tilde{\varepsilon}^{\mathrm{eq}}$ for a planar surface - shape dependence}

One of the more intriguing properties of Eq. (3.17) is the fact that, through $\operatorname{Im} \mathbb{G}(\mathbf{r}, \mathbf{r})$, the effective dielectric function of a homogeneous object is in fact inhomogeneous, unless the whole system is homogeneous (no objects nor boundaries).

To picture this, consider the fluctuations in a linear system,

$$
\left.\left\langle\mathbf{E} \otimes \mathbf{E}^{*}\right\rangle_{\omega}\right|_{\omega} ^{\text {in }}=b(\omega) \mathbb{G}(\operatorname{Im} \mathbb{V}) \mathbb{G}^{*} .
$$

This means the fluctuations at any point $\mathbf{r}$ are due to sources at all other points $\operatorname{Im} \mathbb{V}\left(\mathbf{r}^{\prime}, \mathbf{r}^{\prime}\right)$, which are propagated with the Green's function $\mathbb{G}\left(\mathbf{r}, \mathbf{r}^{\prime}\right)$. So if there is a boundary or absorption between the two points, considering different points $\mathbf{r}$ would give very different fluctuations without changing the noise sources. Since the effective dielectric function depends on these fluctuations through Eq. (3.14), $\tilde{\varepsilon}$ becomes inhomogeneous inside objects, if $\varepsilon$ and $\chi^{(3)}$ are homogeneous. 
For a single flat surface, $\operatorname{Im} \mathbb{G}(\mathbf{r}, \mathbf{r})$ is given in Section B.1 and is plotted in Figures 3.1a, and $3.1 \mathrm{~b}$ for the (linearly) non-absorbing and absorbing case, respectively. Mathematically, of course, subtracting the homogeneous (bulk) solution is problematic in the absorbing case, because it heals the singularities everywhere inside the object, except at the surface. In fact, for frequency-independent materials, we will show below in Section 3.2.4 that this divergence is due to a power law close to the surface.

Figures 3.1 show that, through $\operatorname{Im} \mathbb{G}(\mathbf{r}, \mathbf{r}), \tilde{\varepsilon}$ depends on the shape of objects, meaning the effective dielectric function at a particular point depends on the location of boundaries. However, it is not expected for the interference pattern to survive, unless $\chi^{(3)}\left(-\omega, \omega, \omega^{\prime},-\omega^{\prime}\right)$ has a strong resonance peak at some particular frequency. This can occur, for example, in glasses doped with gold or silver nanospheres, because $\chi^{(3)}$ has a strong relation to the plasmon resonance $[33,36]$.

\subsection{2. $\tilde{\varepsilon}^{\mathrm{eq}}$ for separated objects - combination formulas}

Quite often in physics, predicting something in the absolute sense is difficult and instead it is useful to calculate differences from some reference. This is also true for the effective dielectric function. Specifically, considering a system of separated objects, it will be shown that once $\tilde{\varepsilon}$ is determined for the isolated components (for example through linear response measurements), it is possible to give a well-behaved expression for the effective properties of the full system in terms of the individual measurements. For example, while determining the inhomogeneity of $\tilde{\varepsilon}$ as predicted by Eq. (3.17) and Figure 3.1a could be experimentally challenging, determining a change due to separation proves more viable.

It is important to be clear exactly what is happening theoretically and physically when these measurements in isolation are done (objects moved far from each other). Crucially, it needs to be clarified what properties remain constant, either the bare $\varepsilon$ or the effective $\tilde{\varepsilon}$. It should be noted that the calculation until now did not really address or depend on this, nor was this information required, since the system was considered to be static. Throughout the thesis (and in all the previous work in Refs. $[83,85,86]$ ) we will consider the bare coefficients $\chi^{(n)}$ to describe the "immutable" properties of a particular material or medium. This choice was also implicit in considering the bare coefficients to be homogeneous. It should be understood that this is a physical choice, not a mathematical one, because, barring a microscopic model (not available to us), there is no way to prove one assumption or the other theoretically. In the end, only an experiment can identify a correct choice.

Mathematically, this argument is encoded in the so-called combination formulas. These give a way to express the linear response and potential operators of a system in terms of 


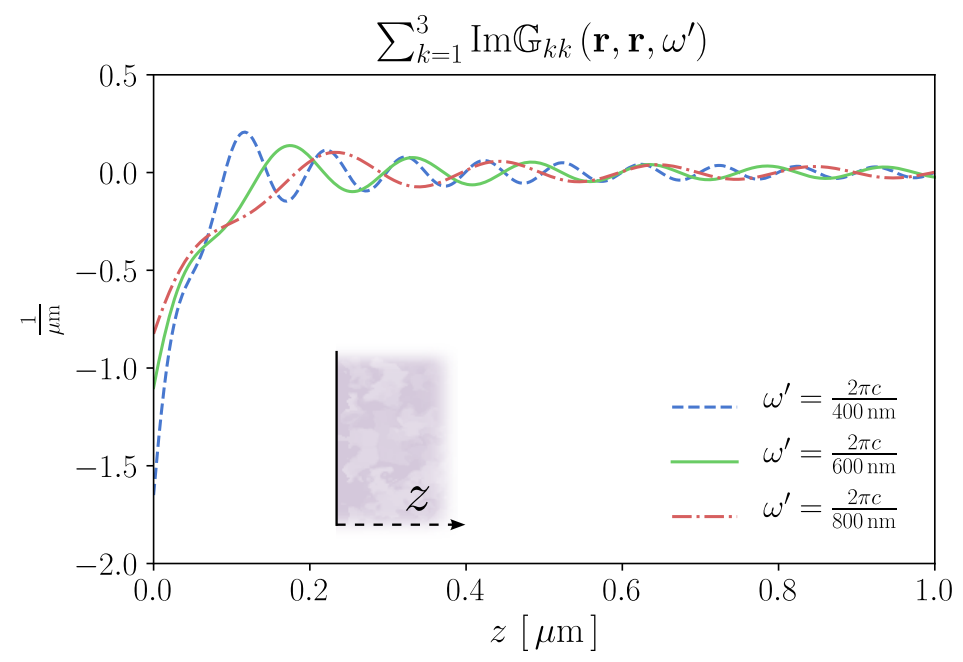

(a)

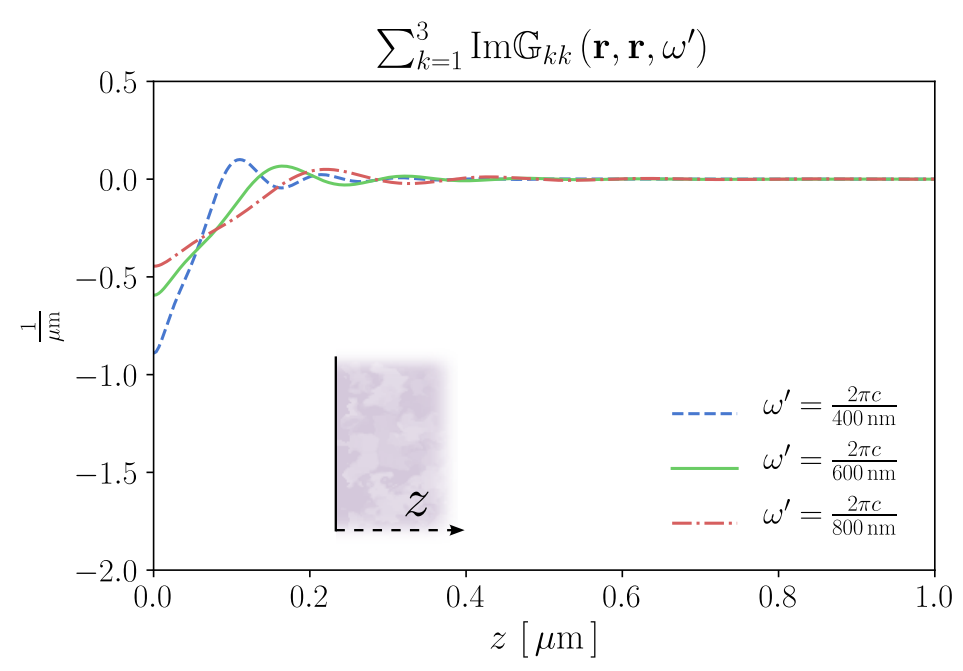

(b)

Figure 3.1.: Inhomogeneity of the effective dielectric function near a surface of a homogeneous isotropic plate for the non-absorbing $\left[\varepsilon\left(\omega^{\prime}\right)=4\right.$, top $]$ and absorbing $\left[\varepsilon\left(\omega^{\prime}\right)=4+i\right.$, bottom $]$ case. By Eq. (3.17), the space-dependence is contained in $\sum_{k} \operatorname{Im} \mathbb{G}_{k k}(\mathbf{r}, \mathbf{r})$, if the nonlinear susceptibility is of the symmetric form $\chi_{i j k l}^{(3)}=\chi^{(3)} \delta_{i j} \delta_{k l}$. 


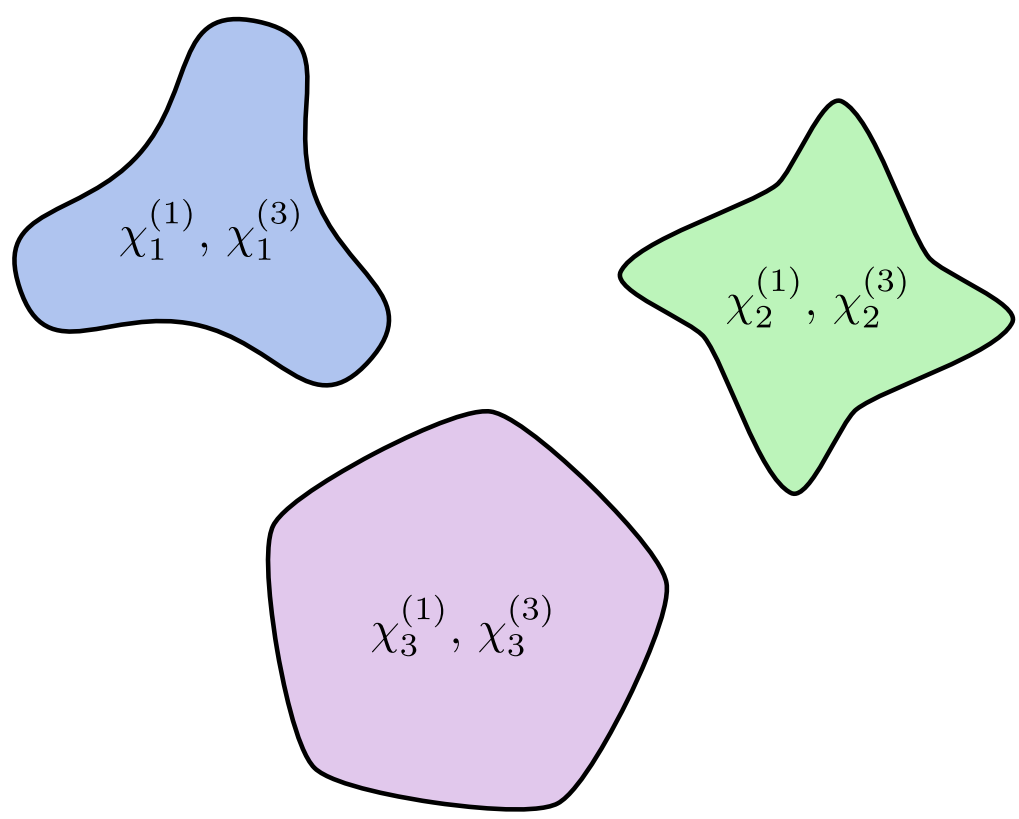

Figure 3.2.: Separation of the bare susceptibilities between spatially separated objects.

the linear responses or potentials of its constituents in isolation. This is important both theoretically, because it gives a way to determine the response of complicated systems, and experimentally, because measuring the response directly might not be practical or even possible (like the effective Fresnel coefficients of a plate when another plate is 10 nanometers away). From the theoretical side, consider the sphere-plate system. The Green's function for a sphere can be conveniently expressed in a partial wave basis [51], while the Green's function for a plate is usually given in plane wave basis with Fresnel coefficients like in Appendix B. For the combined system, with a sphere close to a planar surface, the solution is generally hard to find. A big advantage of FE is the ability to combine together Green's functions of arbitrary systems and perform calculations in a basis-free manner.

\section{Separation of the bare properties of different objects}

As a first step, consider $N$ separated non-overlapping objects, as in Figure 3.2. The region of space occupied by each of them is denoted as $V_{n}$ and the space without any objects as $V_{0}$. Therefore the union $V_{0} \cup V_{1} \cup . . \cup V_{N}$ is the whole space of position vectors $\mathbb{R}^{3}$. The linear and nonlinear susceptibilities of these objects can now be written as

$$
\chi_{n}^{(m)}(\mathbf{r})= \begin{cases}\chi^{(m)}(\mathbf{r}) & \mathbf{r} \in V_{n}, \\ 0 & \text { otherwise. }\end{cases}
$$


The total susceptibility can be recovered by simple summation,

$$
\chi^{(m)}=\sum_{n=1}^{\infty} \chi_{n}^{(m)},
$$

and the bare dielectric functions are simply $\varepsilon_{n}=1+\chi_{n}^{(1)}$. The bare potential operator $\mathbb{V}$ can be also separated,

$$
\begin{aligned}
\mathbb{V} & =\sum_{n=1}^{N} \mathbb{V}_{n}, \\
\mathbb{V}_{n}\left(\mathbf{r}, \mathbf{r}^{\prime}\right)_{i j} & =\delta\left(\mathbf{r}-\mathbf{r}^{\prime}\right) \chi_{n}^{(1)}(\mathbf{r}) .
\end{aligned}
$$

The same holds for the following nonlinear quantities [Eqs. (2.64), (3.20), and (3.17)]:

$$
\begin{aligned}
\mathcal{N} & =\sum_{n=1}^{N} \mathcal{N}_{n}, \\
\mathbb{N} & =\sum_{n=1}^{N} \mathbb{N}_{n}, \\
N & =\sum_{n=1}^{N} N_{n},
\end{aligned}
$$

where the index $n$ denotes that $\chi_{n}^{(3)}$ must be used.

The combination of effective potentials and dielectric functions

For effective potentials, things are slightly more difficult, because the effective potentials are no longer simply additive. Let $\tilde{\mathbb{V}}_{n}^{\text {eq }}$ be the effective potential of object $n$ and $\tilde{\mathbb{V}}^{\text {eq }}$ the full effective potential as per Eq. (3.18),

$$
\begin{aligned}
\tilde{\mathbb{V}}_{n} & =\mathbb{V}_{n}+\mathbb{N}_{n}, \\
\tilde{\mathbb{V}} & =\mathbb{V}+\mathbb{N} .
\end{aligned}
$$




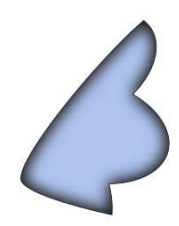

$\tilde{\mathbb{V}}_{1}$

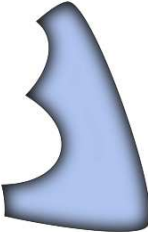

$\tilde{\mathbb{V}}_{2}$

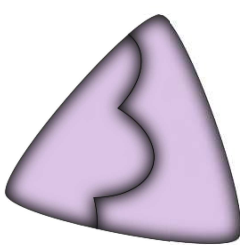

$$
\tilde{\mathbb{V}}^{\prime}=\tilde{\mathbb{V}}_{1}+\tilde{\mathbb{V}}_{2}
$$

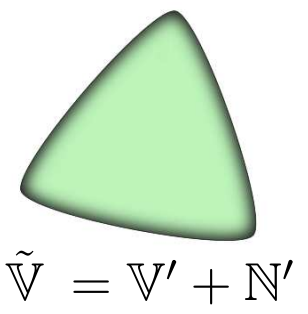

Figure 3.3.: An illustration showing the difference between the naive $\left(\tilde{\mathbb{V}}^{\prime}\right)$ and full $(\tilde{\mathbb{V}})$ effective potentials of the combined object in terms of the effective potentials of its parts $\left(\tilde{\mathbb{V}}_{1}\right.$ and $\left.\tilde{\mathbb{V}}_{2}\right)$, as per Eq. 3.33.

By simple substitution, the effective potential of the combined system can be expressed in terms of $\tilde{\mathbb{V}}_{n}$ :

$$
\begin{aligned}
\tilde{\mathbb{V}}^{\mathrm{eq}} & =\sum_{n=1}^{N}\left(\mathbb{V}_{n}+\mathbb{N}_{n}^{\mathrm{eq}}\right)+\left(\mathbb{N}^{\mathrm{eq}}-\sum_{n=1}^{N} \mathbb{N}_{n}^{\mathrm{eq}}\right) \\
& =\tilde{\mathbb{V}}^{\prime}+\mathbb{N}^{\prime} \\
\mathbb{N}^{\prime} & =\mathbb{N}^{\mathrm{eq}}-\sum_{n=1}^{N} \mathbb{N}_{n}^{\mathrm{eq}}, \\
\tilde{\mathbb{V}}^{\prime} & =\sum_{n=1}^{N} \tilde{\mathbb{V}}_{n}^{\mathrm{eq}} .
\end{aligned}
$$

The quantity on the last line could be called the "naive" effective potential: it is the full potential one would expect, if the nonlinear objects were assumed to be linear (see Figure 3.3). The operator $\mathbb{N}^{\prime}$ could be called the combination correction operator.

As per Eq. (3.20), the analogous equations can be written for the effective dielectric 
function,

$$
\begin{aligned}
\tilde{\varepsilon}^{\mathrm{eq}} & =\tilde{\varepsilon}^{\prime}+N^{\prime}, \\
\tilde{\varepsilon}^{\prime} & =\sum_{n=1}^{N}\left(\tilde{\varepsilon}_{n}^{\mathrm{eq}}-1\right)+1, \\
N^{\prime} & =N^{\mathrm{eq}}-\sum_{n=1}^{N} N_{n}^{\mathrm{eq}} .
\end{aligned}
$$

Using the explicit form for $N^{\text {eq }}$ from Eq. (3.17), and noticing that at any particular coordinate only one $N_{n}^{\text {eq }}$ of the sum contributes, the combination correction to the effective dielectric function $N^{\prime}$ becomes

$$
N_{i j}^{\prime}\left(\mathbf{r} \in V_{n}, \omega\right)=3 \int_{-\infty}^{\infty} \mathrm{d} \omega^{\prime} \chi_{i j k l}^{(3)}\left(\mathbf{r} ;-\omega, \omega, \omega^{\prime},-\omega^{\prime}\right) b\left(\omega^{\prime}\right) \operatorname{Im}\left[\mathbb{G}-\mathbb{G}_{n}\right]_{k l}\left(\mathbf{r}, \mathbf{r} ; \omega^{\prime}\right),
$$

where $\mathbb{G}_{n}=\left(\mathbb{H}_{0}-\mathbb{V}_{n}\right)^{-1}$ is the Green's function with only object $n$ present. Note that since they appear alongside $\chi^{(3)}$, the linear responses $\tilde{\mathbb{G}}$ and $\tilde{\mathbb{G}}_{n}$ could be used just as well.

There are three big benefits to using the effective dielectric function (same applies to the potential operator $\tilde{\mathbb{V}}$ ) defined through the combination formulas Eqs. (3.36) and (3.39) as opposed to the definitions from bare quantities Eqs. (3.14) and (3.17):

1. The effective dielectric function at a point $\mathbf{r} \in V_{n}$ given in Eq. 3.36 is completely specified by measurable quantities: the dielectric function measured in isolation $\left(\tilde{\varepsilon}_{n}\right)$ and the difference between full and isolated absorption, $\operatorname{Im}\left[\tilde{\mathbb{G}}_{-}-\tilde{\mathbb{G}}_{n}\right](\mathbf{r}, \mathbf{r})$.

2. The difference $\operatorname{Im}\left[\tilde{\mathbb{G}}-\tilde{\mathbb{G}}_{n}\right](\mathbf{r}, \mathbf{r})$ removes the divergence that was discussed above in Section 3.2. This means we can treat absorbing materials with ease. ${ }^{1}$

3. Since $\tilde{\varepsilon}^{\prime}$ is defined through isolated measurements, all distance dependence must be contained in $N^{\prime}$ (or, equivalently, $\mathbb{N}^{\prime}$ ). This will be used to calculate deviations from the Lifshitz formula below.

The only downside is that this method cannot be applied to determine to $\tilde{\varepsilon}^{\mathrm{eq}}$ of a single absorbing surface (see Figure 3.1a).

\footnotetext{
${ }^{1}$ This must also be the case as a consequence of the first point: Eqs. (3.33) and (3.36) relate only physically observable quantities, which must be finite.
} 

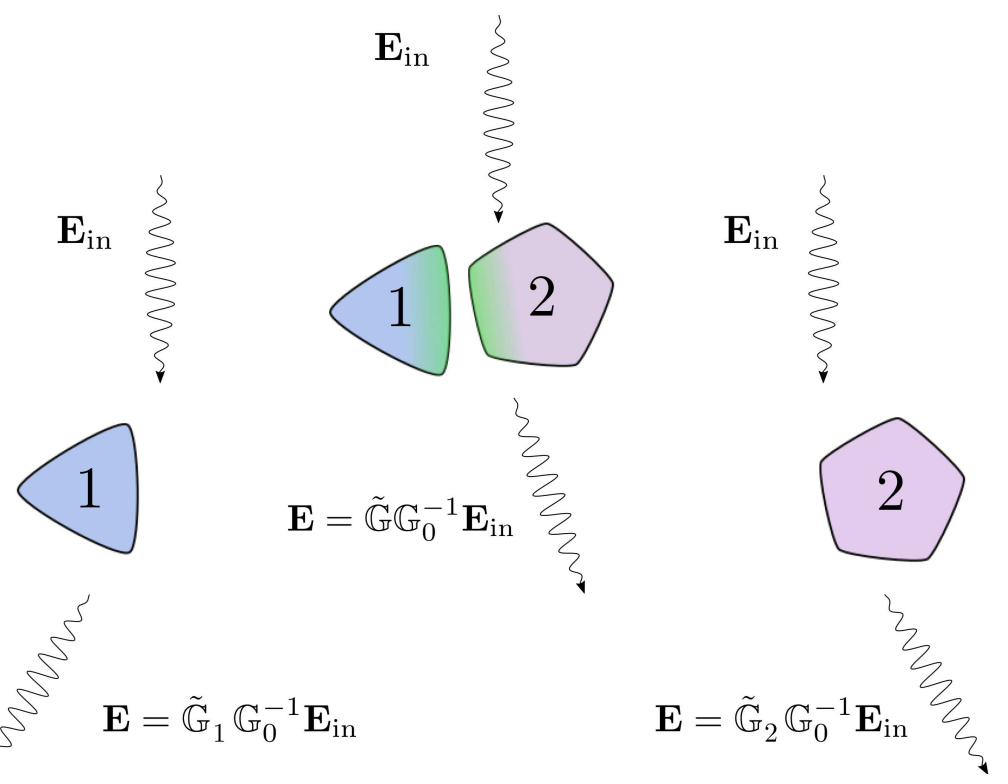

Figure 3.4.: Experimental configurations for linear response measurements. For objects with a nonlinear response, the known combination formula Eq. (3.43) does not give the correct linear response of the combined system.

\section{The combination of linear responses}

More important for calculations in FE, is the combination formula for linear responses. The Green's function corresponding to the naive potential in Eq. 3.35 is

$$
\tilde{\mathbb{G}}^{\prime}=\left(\mathbb{H}_{0}-\tilde{\mathbb{V}}^{\prime}\right)^{-1} .
$$

This is the linear response one expects if the system is assumed to be linear and the potentials of individual objects are measured. The full linear response is

$$
\tilde{\mathbb{G}}=\tilde{\mathbb{G}}^{\prime}+\tilde{\mathbb{G}}^{\prime} \mathbb{N}^{\prime} \tilde{\mathbb{G}}^{\prime}
$$

where the combination correction $\mathbb{N}^{\prime}$ is given by Eq. (3.34) above.

Since we are mostly interested in the combination of the linear responses of two objects (or groups of objects), for example moving one object while keeping the rest fixed, we calculate this case explicitly. The two bodies have potentials $\tilde{\mathbb{V}}_{1}$ and $\tilde{\mathbb{V}}_{2}$ with linear responses $\widetilde{\mathbb{G}}_{1}$ and $\widetilde{\mathbb{G}}_{2}$ (measured in isolation), as illustrated in Figure 3.4. The naive 


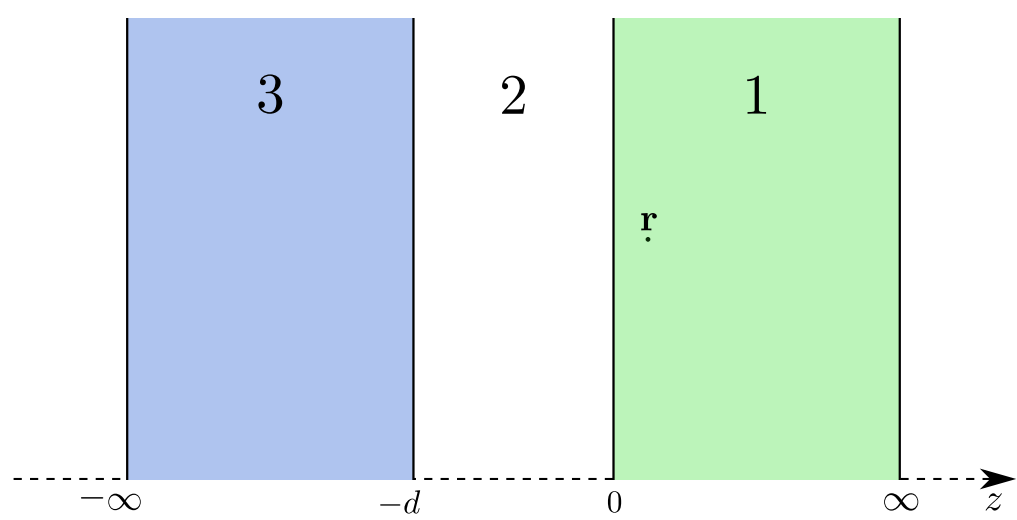

Figure 3.5.: A system of semi-infinite parallel plates, separated by vacuum (2). Plate 1 is considered fixed while the position of plate 3 is varied.

combination formula would be

$$
\begin{gathered}
\tilde{\mathbb{V}}^{\prime}=\tilde{\mathbb{V}}_{1}^{\text {eq }}+\tilde{\mathbb{V}}_{2}^{\text {eq }}, \\
\tilde{\mathbb{G}}^{\prime}=\tilde{\mathbb{G}}_{2}\left(\tilde{\mathbb{G}}_{1}+\tilde{\mathbb{G}}_{2}+\tilde{\mathbb{G}}_{1} \mathbb{G}_{0}^{-1} \tilde{\mathbb{G}}_{2}\right)^{-1} \tilde{\mathbb{G}}_{1} .
\end{gathered}
$$

The last formula is very widely used in linear FE [51] and is in fact the basis for the Lifshitz equation (C.13), while the first is considered trivial. The full potential and response of the combined system are, however, different for nonlinear systems,

$$
\begin{aligned}
\tilde{\mathbb{V}} & =\tilde{\mathbb{V}}_{n}^{\prime}+\mathbb{N}^{\prime}, \\
\tilde{\mathbb{G}} & =\tilde{\mathbb{G}}^{\prime}+\tilde{\mathbb{G}}^{\prime} \mathbb{N}^{\prime} \tilde{\mathbb{G}}^{\prime}, \\
\mathbb{N}^{\prime} & =\left(\mathbb{N}-\mathbb{N}_{1}^{\mathrm{eq}}-\mathbb{N}_{2}^{\mathrm{eq}}\right) .
\end{aligned}
$$

\subsection{3. $\tilde{\varepsilon}^{\mathrm{eq}}$ for two parallel surfaces - distance dependence}

Using Eq. (3.39) for separated objects, it is no longer necessary to restrict the calculations to real (non-absorbing) $\varepsilon$. Instead of considering the full effective dielectric function $\tilde{\varepsilon}$, it is possible study the dependence of the effective properties on the separation of the objects, which is more convenient and interesting, because this behavior is absent for objects with purely linear dielectric properties.

A simple example to consider is the case of two parallel semi-infinite surfaces separated by distance $d$ (see Figure 3.5). The effective dielectric function inside slab 1 (which is 
kept in place) can be written with the help of Eqs. (3.36) and (3.39),

$$
\begin{aligned}
\tilde{\varepsilon}^{\mathrm{eq}}(d) & =\tilde{\varepsilon}^{\mathrm{eq}}(d \rightarrow \infty)+N^{\prime}(d), \\
N_{i j}^{\prime}(\mathbf{r}, \omega, d) & =3 \int_{-\infty}^{\infty} \mathrm{d} \omega^{\prime} \chi_{i j k l}^{(3)}\left(\mathbf{r} ;-\omega, \omega, \omega^{\prime},-\omega^{\prime}\right) b\left(\omega^{\prime}\right) \\
& \times\left[\operatorname{Im} \mathbb{G}_{k l}\left(d ; \mathbf{r}, \mathbf{r} ; \omega^{\prime}\right)-\operatorname{Im} \mathbb{G}_{k l}\left(d \rightarrow \infty ; \mathbf{r}, \mathbf{r} ; \omega^{\prime}\right)\right] .
\end{aligned}
$$

If the plate is homogeneous, meaning $\varepsilon$ and $\chi^{(3)}$ are constant inside the plate, then the $\mathbf{r}$ and $d$ dependence is contained completely in $\operatorname{Im} \mathbb{G}(\mathbf{r}, \mathbf{r})$, as for the case of the single plate in Section 3.2.1.

Numerical results for $\operatorname{Im} \mathbb{G}(d, \mathbf{r}, \mathbf{r})-\operatorname{Im} \mathbb{G}(d \rightarrow \infty, \mathbf{r}, \mathbf{r})$ for non-absorbing $(\varepsilon=4)$ and absorbing $(\varepsilon=4+i)$ cases are shown in Figures 3.6a and 3.6b, respectively. Note that they exhibit a similar interference pattern as the single slab case in Figures 3.1, which now undergoes a phase shift with $d$. For the non-absorbing case this pattern persists throughout the material, while in the absorbing case the bulk value is approached beyond the skin depth. As before, only the $\omega^{\prime}=\frac{2 \pi c}{400 \mathrm{~nm}}$ component is shown, so unless $\chi^{(3)}$ has a strong resonance, the interference at different frequencies would cancel.

By design, $\tilde{\varepsilon}^{\mathrm{eq}}(d)$ at large separations would approach the single plate result. On the other hand, the distance dependence at small $d$ affects directly the Casimir force as given in Refs. $[83,85]$, as we will see in the next section. In particular, the well-known distance power laws of the Lifshitz formula must be modified for nonlinear objects.

\subsubsection{Bulk, surface, and distance contributions for homogeneous objects}

While Eq. (3.47) is useful on its own, this method can be used to identify and investigate all the separate contributions to the effective dielectric function. Consider again the situation of two parallel plates as in Figure 3.5, but let the plates consist of the same, homogeneous material. The dielectric function in plate 1 is given by Eqs. (3.14) and (3.17) (using the coordinate $z=\hat{z} \cdot \mathbf{r}$, because of translation invariance in the xy-plane),

$$
\begin{aligned}
\tilde{\varepsilon}_{i j}^{\mathrm{eq}}(d, z>0, \omega) & =\varepsilon_{i j}(\omega)+N_{i j}^{\mathrm{eq}}(d, z, \omega), \\
N_{i j}^{\mathrm{eq}}(d, z, \omega) & =3 \int_{-\infty}^{\infty} \mathrm{d} \omega^{\prime} \chi_{i j k l}^{(3)}\left(-\omega, \omega, \omega^{\prime},-\omega^{\prime}\right) b\left(\omega^{\prime}\right) \operatorname{Im} \mathbb{G}_{k l}\left(d ; \mathbf{r}, \mathbf{r} ; \omega^{\prime}\right) .
\end{aligned}
$$

By setting up the experiment at different distances, we can identify different contributions. 


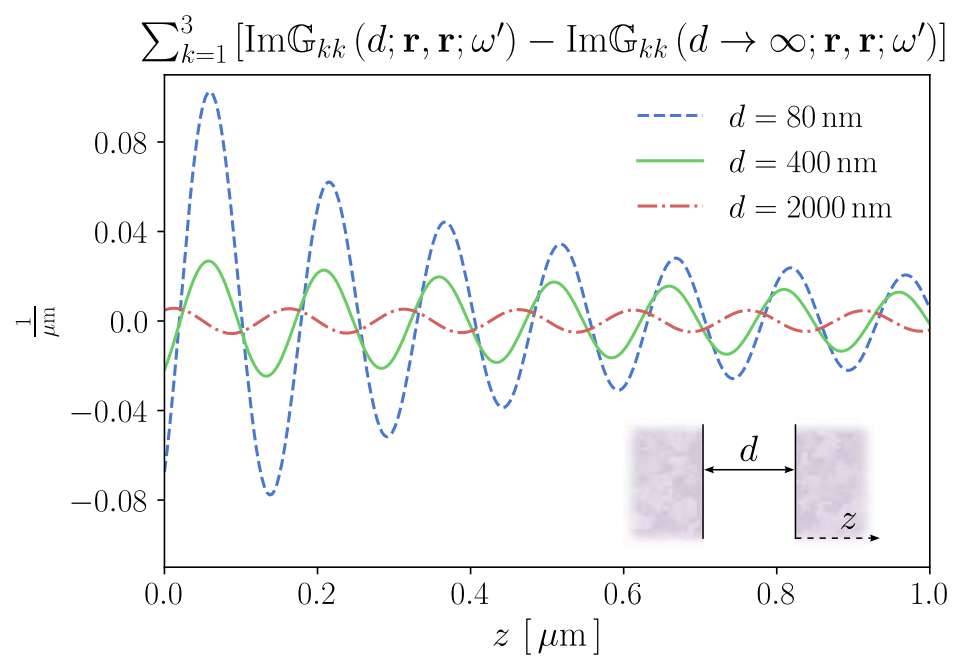

(a)

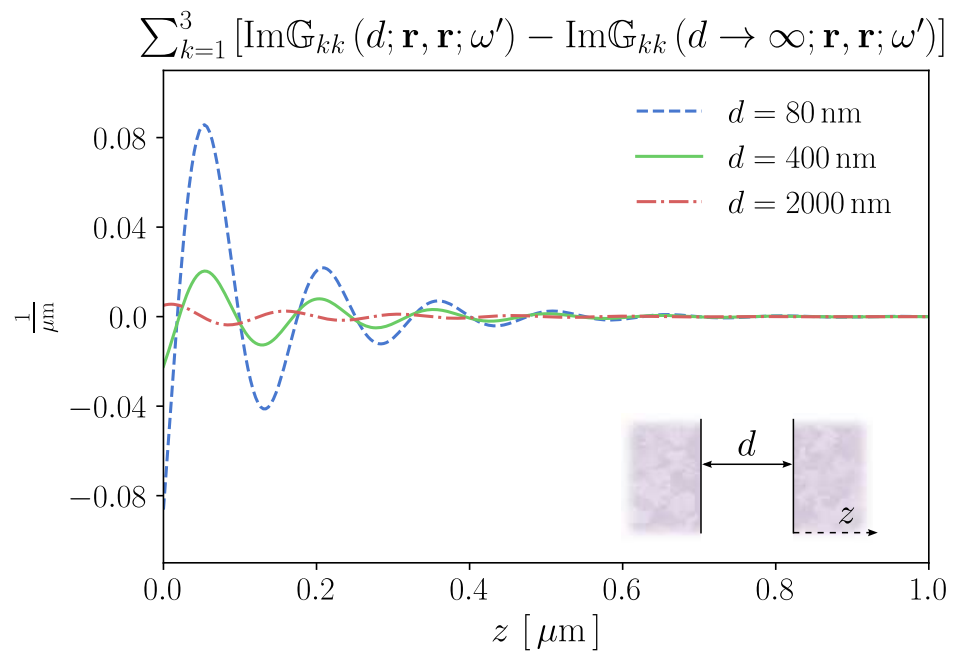

(b)

Figure 3.6.: Dependence of the effective dielectric function on the distance between two homogeneous isotropic plates, for the non-absorbing $\left[\varepsilon\left(\omega^{\prime}\right)=4\right.$, top] and absorbing $\left[\varepsilon\left(\omega^{\prime}\right)=4+i\right.$, bottom] case. These plots are calculated for $\omega^{\prime}=\frac{2 \pi c}{600 \mathrm{~nm}}$, which corresponds to a resonance in the optical region and determines the decay length into the material (skin depth). 


\section{Bulk properties}

At $d \rightarrow 0$, we must obtain a homogeneous effective epsilon for a bulk material

$$
\tilde{\varepsilon}_{i j}^{\text {bulk }}(\omega)=\varepsilon_{i j}(\omega)+N^{\mathrm{eq}}(d \rightarrow 0, \omega) .
$$

As mentioned in Section 3.2 and calculated in Section (B.3), the equal point Green's function in $N^{\mathrm{eq}}(d \rightarrow 0)$ diverges if there is absorption. However, being a result of experiment, $\tilde{\varepsilon}_{i j}^{\text {bulk }}(\omega)$ must be finite (it is very common to renormalize bulk terms in field theories [40]). Therefore terms relative to $\tilde{\varepsilon}^{\text {bulk }}$ will be considered, which sidesteps this problem. Measuring the bare $\varepsilon$ experimentally is in any case not possible.

\section{Surface contributions}

In the limit $d \rightarrow \infty$, we obtain the effective dielectric function for an isolated plate,

$$
\begin{aligned}
\tilde{\varepsilon}_{i j}^{\text {single }}(z, \omega) & =\varepsilon_{i j}(\omega)+N^{\mathrm{eq}}(d \rightarrow \infty, z, \omega) \\
& =\tilde{\varepsilon}_{i j}^{\text {bulk }}(\omega)+N^{\text {surface }}(z, \omega), \\
N^{\text {surface }}(z, \omega) & =\tilde{\varepsilon}_{i j}^{\text {single }}(z, \omega)-\tilde{\varepsilon}_{i j}^{\text {bulk }}(\omega) \\
& =N^{\mathrm{eq}}(d \rightarrow \infty)-N^{\mathrm{eq}}(d \rightarrow 0) \\
& =3 \int_{-\infty}^{\infty} \mathrm{d} \omega^{\prime} \chi_{i j k l}^{(3)}\left(-\omega, \omega, \omega^{\prime},-\omega^{\prime}\right) b\left(\omega^{\prime}\right) \\
& \times \operatorname{Im}\left[\mathbb{G}_{k l}\left(d \rightarrow \infty ; \mathbf{r}, \mathbf{r} ; \omega^{\prime}\right)-\mathbb{G}_{k l}\left(d \rightarrow 0, \mathbf{r}, \mathbf{r} ; \omega^{\prime}\right)\right] .
\end{aligned}
$$

As mentioned above, $\tilde{\varepsilon}^{\text {eq }}$ diverges for a single plate at the surface if there is any absorption, but we can also show this explicitly.

Appendix B contains an explicit calculation for the imaginary part of the difference of Green's functions [Eq. (B.18)]. Expanding the hyperbolic cotangents at high and low temperatures,

$$
\begin{aligned}
\operatorname{coth}\left(\frac{1}{z} \frac{\hbar \omega}{2 k_{\mathrm{B}} T}\right) & \approx 1+\mathcal{O}\left(T^{-1}\right), \\
\operatorname{coth}\left(\frac{1}{z} \frac{\hbar \omega}{2 k_{\mathrm{B}} T}\right) & \approx 2 z \frac{k_{\mathrm{B}} T}{\hbar \omega}+\mathcal{O}\left(T^{2}\right),
\end{aligned}
$$

and assuming no further $\omega$-dependence in $\chi^{(1)}$ or $\chi^{(3)}$, we can obtain the following 
power laws in the corresponding limits,

$$
\begin{aligned}
& N_{i j}^{\text {surface }}(\mathbf{r}, \omega, T \rightarrow 0)=\frac{1}{z^{4}} \frac{3 \hbar}{16 \pi^{2} \varepsilon_{0}} \chi_{i j k l}^{(3)}(\mathbf{r}) \operatorname{Re} \int_{0}^{\infty} \mathrm{d} \omega^{\prime} \frac{\omega^{\prime 2}}{c^{2}} \int_{0}^{\infty} \mathrm{d} q_{\|} e^{2 i p_{1}^{\prime}} \frac{q_{\|}}{p_{1}^{\prime}} \\
& \times\left(\begin{array}{ccc}
\mathcal{F}_{12}^{\prime s}-\mathcal{F}_{12}^{p \prime} \frac{p_{1}^{\prime 2}}{k_{1}^{\prime 2}} & 0 & 0 \\
0 & \mathcal{F}_{12}^{\prime s}-\mathcal{F}_{12}^{\prime p \frac{p_{1}^{\prime 2}}{k_{1}^{\prime 2}}} & 0 \\
0 & 0 & 2\left(\mathcal{F}_{12}^{\prime p}-1\right) \frac{q_{\|}^{2}}{k_{1}^{\prime 2}}
\end{array}\right)_{k l}, \\
& N_{i j}^{\text {surface }}(\mathbf{r}, \omega, T \rightarrow \infty)=\frac{1}{z^{3}} \frac{3 k_{\mathrm{B}} T}{8 \pi^{2} \varepsilon_{0} c} \chi_{i j k l}^{(3)}(\mathbf{r}) \operatorname{Re} \int_{0}^{\infty} \mathrm{d} \omega^{\prime} \frac{\omega^{\prime}}{c} \int_{0}^{\infty} \mathrm{d} q_{\|} e^{2 i p_{1}^{\prime}} \frac{q_{\|}}{p_{1}^{\prime}} \\
& \times\left(\begin{array}{ccc}
\mathcal{F}_{12}^{\prime s}-\mathcal{F}_{12}^{p \prime} \frac{p_{1}^{\prime 2}}{k_{1}^{\prime 2}} & 0 & 0 \\
0 & \mathcal{F}_{12}^{\prime s}-\mathcal{F}_{12}^{\prime p \frac{p_{1}^{2}}{k_{1}^{\prime 2}}} & 0 \\
0 & 0 & 2\left(\mathcal{F}_{12}^{\prime p}-1\right) \frac{q_{\|}^{2}}{k_{1}^{\prime 2}}
\end{array}\right)_{k l},
\end{aligned}
$$

where the various quantities are defined in Appendix B. It can be seen that the quantum and thermal limits of the surface contributions obey the power laws $z^{-4}$ and $z^{-3}$, respectively. This is a sign of the breakdown of the field theory, which does not hold close to or lower than atomic scales (or the scale of building blocks for metamaterials). A common remedy is to introduce an ultraviolet cutoff of the frequencies. In our case we recognize that even though $N^{\text {surface }}$ obeys a power law for large $z$, it must be finite near $z \rightarrow 0$ due to physical considerations.

Compare this result with the Figure 3.1: a wide band $\chi^{(3)}$ leads to a power law, but a resonant $\chi^{(3)}$ gives oscillations.

\section{Distance dependence}

Finally, the effective dielectric function with both plates present can be written as

$$
\begin{aligned}
\tilde{\varepsilon}_{i j}^{\text {double }}(d, z, \omega) & =\varepsilon_{i j}(\omega)+N_{i j}^{\mathrm{eq}}(d, z, \omega) \\
& =\tilde{\varepsilon}_{i j}^{\text {single }}(z, \omega)+N^{\text {dist }}(d, z, \omega) \\
& =\tilde{\varepsilon}_{i j}^{\text {bulk }}(\omega)+N^{\text {surface }}(z, \omega)+N^{\text {dist }}(d, z, \omega),
\end{aligned}
$$


where the distance contribution is already familiar from Eq. (3.48),

$$
\begin{aligned}
N^{\mathrm{dist}}(d, z, \omega) & =\tilde{\varepsilon}_{i j}^{\mathrm{eq}}(d, z>0, \omega) \\
& =N_{i j}^{\mathrm{eq}}(d, z, \omega)-N^{\mathrm{eq}}(d \rightarrow \infty) .
\end{aligned}
$$

In the next chapter this description will be amended with a term taking into account temperature differences [see Eq. (4.27)].

\subsection{Example - equilibrium Casimir force}

With the effective dielectric function at hand, one could think of calculating the Casimir directly from the famous Lifshitz formula [59] (given in Eq. C.13). The problem is that the classic Lifshitz equation assumes that the plates are homogeneous, taking only a single, frequency-dependent dielectric function as a parameter, whereas we found instead that the effective dielectric function is inhomogeneous inside otherwise homogeneous objects.

This problem was explored extensively in Ref. [83], with a relatively convenient form for the nonlinear contribution [Eq. (C.14)] given in Ref. [85] a year later. We refrain from carrying out this derivation again here and rather concentrate on the basis of this calculation and the insights from the analysis in Ref. [85].

\subsubsection{Modified power laws for frequency-independent materials}

\section{Simplifications and geometry}

The exact distance dependence of the nonlinear Casimir force is complicated, not least because it depends on the generally unknown functional forms of $\varepsilon(\omega)$ and $\chi^{(3)}\left(-\omega, \omega, \omega^{\prime},-\omega^{\prime}\right)$. This applies to both the linear and nonlinear parts. However, since the Casimir force is a wide-band effect (all frequencies contribute in principle), a good qualitative result can be obtained by considering frequency-independent materials, as in Section 3.2.4.

For the sake of simplicity, it is further assumed that the materials are non-absorbing (so both $\varepsilon$ and $\chi^{(3)}$ are real) and isotropic (see Section 2.2.1). In the simplest case, the material properties can be written as

$$
\begin{aligned}
\varepsilon_{i j}(\omega) & =\delta_{i j} \varepsilon \\
\chi_{i j k l}^{(3)}\left(-\omega, \omega, \omega^{\prime},-\omega^{\prime}\right) & =\left(\delta_{i j} \delta_{k l}+\delta_{i k} \delta_{j l}+\delta_{i l} \delta_{j k}\right) \chi^{(3)} .
\end{aligned}
$$




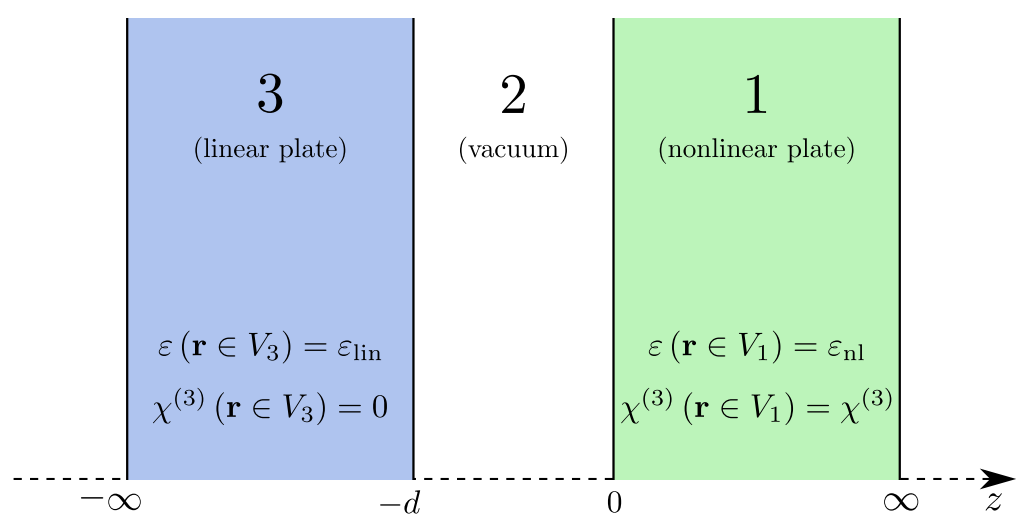

Figure 3.7.: System of one nonlinear (1) and one linear (3) plate, separated by vacuum (2). Due to working in leading order in $\chi^{(3)}$, it is sufficient to consider only a single nonlinear plate at a time for calculating the Casimir force. The nonlinear contributions from either plate are additive.

As per Figure 3.7, the plate at $z<-d$ is characterized by the dielectric constant $\varepsilon_{\text {lin }}$ and the plate at $z>0$ by $\varepsilon_{\mathrm{nl}}$ and $\chi^{(3)}$. Note that it is trivial to make both plates nonlinear, since the contributions are additive.

\section{Scaling argument and power laws}

With these simplifications, it is relatively easy to obtain the distance power laws from Eqs. (C.13) and (C.14). The key is to scale the integration by distance, such that $\omega, \omega^{\prime}, q, q^{\prime} \rightarrow \frac{\omega}{d}, \frac{\omega^{\prime}}{d}, \frac{q}{d}, \frac{q^{\prime}}{d}$. Together with the expansion of the hyperbolic cotangents at high and low temperatures, $\operatorname{coth}\left(\frac{1}{d} \frac{\hbar \omega}{2 k_{\mathrm{B}} T}\right) \approx 1$ and $\operatorname{coth}\left(\frac{1}{d} \frac{\hbar \omega}{2 k_{\mathrm{B}} T}\right) \approx 2 d \frac{k_{\mathrm{B}} T}{\hbar \omega}$, respectively, the following expressions can be obtained,

$$
\begin{aligned}
P^{T \rightarrow 0} & =\frac{\hbar c}{d^{4}} I_{\operatorname{lin}}^{T \rightarrow 0}+\frac{\chi^{(3)}}{\varepsilon_{0}}\left(\frac{\hbar c}{d^{4}}\right)^{2} I_{\mathrm{nl}}^{T \rightarrow 0}, \\
P^{T \rightarrow \infty} & =\frac{k_{\mathrm{B}} T}{d^{3}} I_{\operatorname{lin}}^{T \rightarrow \infty}+\frac{\chi^{(3)}}{\varepsilon_{0}}\left(\frac{k_{\mathrm{B}} T}{d^{3}}\right)^{2} I_{\mathrm{nl}}^{T \rightarrow \infty},
\end{aligned}
$$

where the different $I$ represent the remaining dimensionless integrals, which are independent of distance or temperature (similar to Eqs. (3.56) and (3.58)). While $\frac{1}{d^{3}}$ and $\frac{1}{d^{4}}$ are the well-known power laws of the Casimir force in the quantum and thermal limits, the $\frac{1}{d^{6}}$ and $\frac{1}{d^{8}}$ forces are new. They are caused by the distance dependence of the effective dielectric function of the nonlinear plate (see Eq. (3.48)).

These integrals $I$ are shown in Figures 3.8a and 3.8b for various combinations of the dielectric constants of either plate. Note that the dielectric constant of the linear plate, 


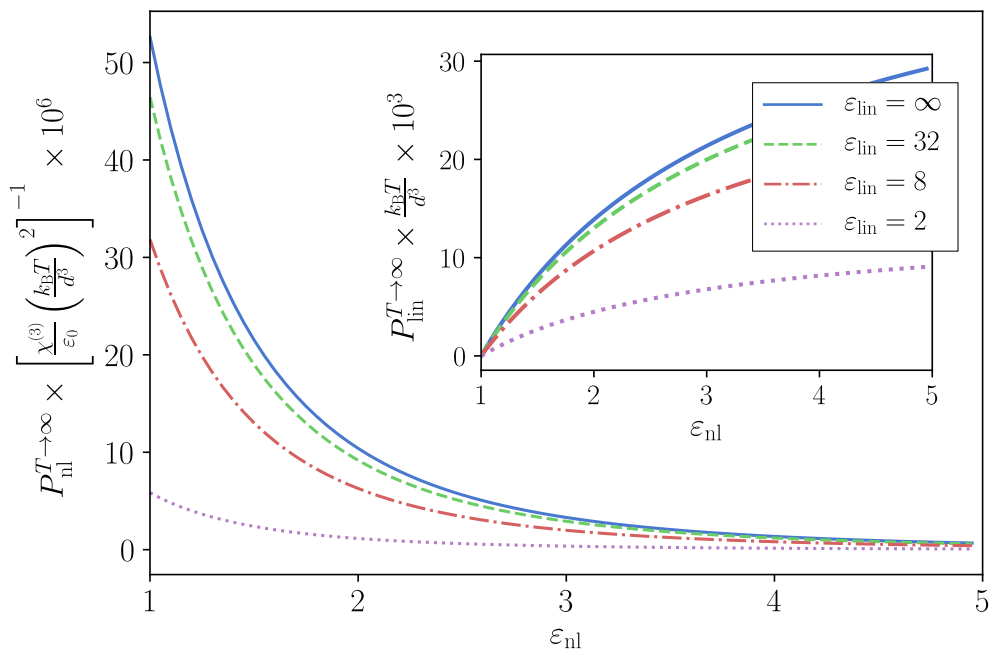

(a)

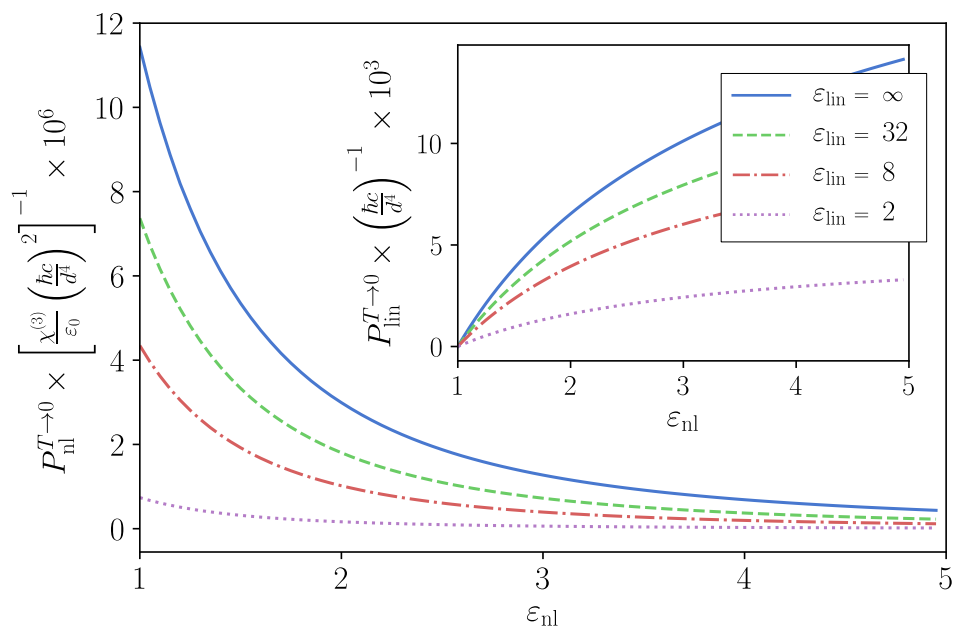

(b)

Figure 3.8.: Casimir force in the thermal $(T \rightarrow \infty)$ and quantum $(T \rightarrow 0)$ limit as per Eqs. 3.63 and 3.64 as a function of the dielectric constant $\varepsilon_{\mathrm{nl}}$ of the nonlinear plate (see Figure 3.7). The main graphs show the nonlinear contributions $\left(I_{\mathrm{nl}}^{T \rightarrow 0}\right.$ and $\left.I_{\mathrm{nl}}^{T \rightarrow \infty}\right)$ while the insets show the linear parts $\left(I_{\operatorname{lin}}^{T \rightarrow 0}\right.$ and $\left.I_{\mathrm{lin}}^{T \rightarrow \infty}\right)$. 


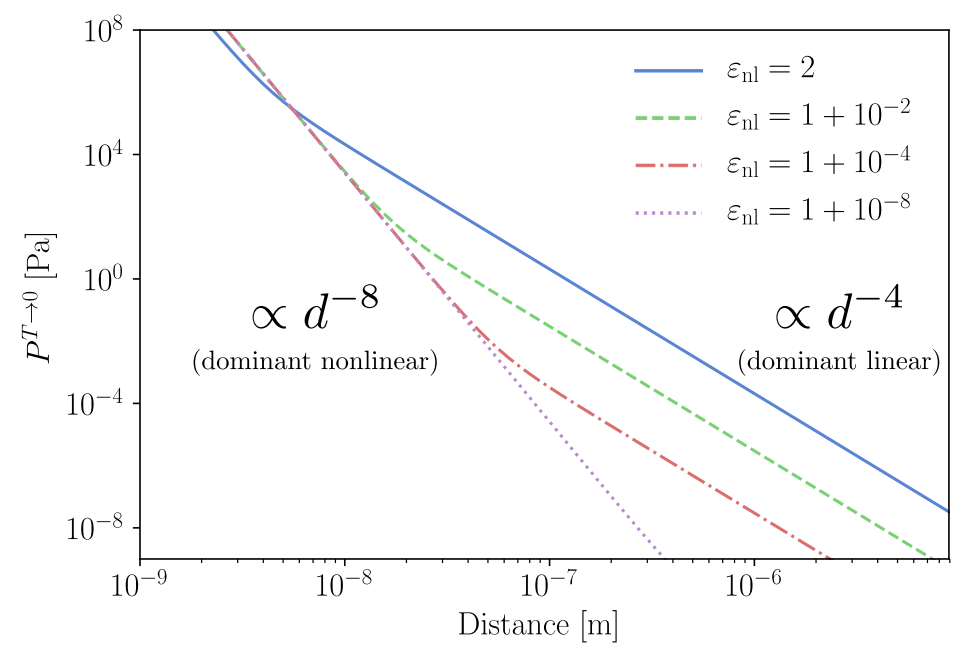

Figure 3.9.: Casimir force between a perfect mirror and a nonlinear slab with a dielectric constant $\varepsilon_{\mathrm{nl}}$ as labeled and nonlinear susceptibility $\chi^{(3)}=2 \times 10^{-16} \frac{\mathrm{m}^{2}}{\mathrm{~V}^{2}}$ (corresponding roughly to glass infused with silver nanoparticles).

$\varepsilon_{\text {lin }}$, can be increased to infinity (the case of a perfect mirror), while the same is not true for the corresponding property of the nonlinear plate $\varepsilon_{n 1}$. Increasing the latter will still increase of the conventional $\frac{1}{d^{3}}$ and $\frac{1}{d^{4}}$ forces, but decrease the $\frac{1}{d^{6}}$ and $\frac{1}{d^{8}}$ forces. In fact the maximum "nonlinear" pressure is obtained if $\varepsilon_{\text {lin }} \rightarrow \infty$ and $\varepsilon_{\mathrm{nl}} \rightarrow 1$. That way no light is "lost" through transmission in the linear plate, or through internal and external reflection from the nonlinear plate. In the limit $\varepsilon_{\mathrm{nl}} \rightarrow 1$, the nonlinear contribution vanishes, because all the fluctuations from the other plate can no longer penetrate the surface and undergo nonlinear interactions.

\section{Crossover}

Figure 3.9 shows the Casimir force in the quantum limit given by Eq. (3.63) for the case $\varepsilon_{\text {lin }} \rightarrow \infty$ (a perfect mirror). As expected, the pressure has the well-known $\frac{1}{d^{4}}$ behavior for large separations and crosses over to $\frac{1}{d^{8}}$ for small separations.

An optimistic nonlinear susceptibility $\chi^{(3)}=2 \times 10^{-16} \frac{\mathrm{m}^{2}}{\mathrm{~V}^{2}}$ was used, corresponding to off-resonant nonlinearity of glass fused with silver nanoparticles [41]. Nevertheless the crossover occurs at around $5-10 \mathrm{~nm}$ for realistic materials with $\varepsilon_{\mathrm{nl}} \sim 2$, which is very small, but potentially achievable with current experimental methods.

By reducing $\varepsilon_{\mathrm{nl}}$, the crossover distance can potentially be increased to infinity. While this seems hard to achieve, the same effect (reducing the linear contribution) could be reached with index-matched materials or coating $[87,16,98]$. 


\subsubsection{Force between an invisible plate and a perfect mirror}

Based on Figure 3.9, it is interesting to consider the limit where the linear contributions vanish. Thus we consider a plate that is invisible (in isolation no light is reflected) in front of a perfect mirror. The linear contribution to the force [Eq. (C.13)] vanishes, while Eq. (C.14) simplifies to

$$
\begin{aligned}
P & =\frac{3}{2^{8} \pi^{4}} \varepsilon_{0} \operatorname{Re} \iiint \int_{0}^{\infty} \mathrm{d} \omega \mathrm{d} \omega^{\prime} \mathrm{d} q \mathrm{~d} q^{\prime} \chi^{(3)}\left(-\omega, \omega, \omega^{\prime},-\omega^{\prime}\right) \\
& \times a(\omega) a\left(\omega^{\prime}\right)\left[\frac{e^{2 i\left(p+p^{\prime}\right) d}}{\left(p+p^{\prime}\right) p^{\prime}}+\frac{e^{2 i\left(p-p^{* *}\right) d}}{\left(p-p^{\prime *}\right) p^{\prime}}\right] \\
& \times \frac{q q^{\prime}}{k^{2} k^{\prime 2}}\left[k^{2}\left(4 k^{\prime 2}-3 q^{\prime 2}\right)-q^{2}\left(6 k^{\prime 2}-7 q^{\prime 2}\right)\right]
\end{aligned}
$$

where $k=\frac{\omega}{c}$ and $p=\sqrt{k^{2}-q^{2}}$.

The "invisibility" condition for the nonlinear plate is required in isolation, $\tilde{\varepsilon}_{\mathrm{nl}}(d \rightarrow \infty)=1$. A nonzero force will still be observed, because the previously invisible plate becomes reflecting near the mirror due to Eq. (3.47). It should also be noted that the condition $\tilde{\varepsilon}_{\mathrm{nl}}(d \rightarrow \infty)=1$ is far from innocuous and cannot be achieved with homogeneous media (see Eqs. (3.52) and (3.56)).

\subsection{Summary}

By taking the stochastic and effective Helmholtz equations obtained in the previous chapter and applying the FDT in equilibrium, the noise and fluctuations could be determined explicitly. We also confirmed that the calculation agrees with the Rytov theory, provided the effective potential or dielectric function is used.

The effective dielectric function $\tilde{\varepsilon}$ was investigated in great detail, as a quantity directly affected by fluctuations. Since the latter depend greatly on the rest of the system, so too does $\tilde{\varepsilon}$ become "aware" of the surroundings (as opposed to linear systems, where the dielectric function is purely a local property). We saw that $\tilde{\varepsilon}$ becomes inhomogeneous for otherwise homogeneous objects, because it depends on the distance to the surfaces (vacuum fluctuations for example limited by the skin depth). This dependence is a power law for frequency-independent materials or results in an interference pattern if the nonlinear susceptibility $\chi^{(3)}$ is highly resonant.

The results were also applied to the classic problem of the Casimir force between parallel plates. Specifically, we saw that at very close distances the ordinary $d^{-4}\left(d^{-3}\right)$ power 
3. Fluctuational electrodynamics for nonlinear materials in equilibrium

law crosses over to $d^{-8}\left(d^{-6}\right)$ for the $T \rightarrow 0(T \rightarrow \infty)$ limit. For experimental detection, the linear component can be minimized by considering a plate that in isolation has a nonlinear, but no linear response. 


\section{Thermal imbalance in nonlinear systems and radiative heat transfer}

In the previous chapter we investigated electromagnetic fluctuations in equilibrium systems, and, even though some assumptions were needed (that $\langle\mathbf{F}\rangle=0$ and that the bare electromagnetic potential $\mathbb{V}$ is constant), the theory is still well grounded through the FDT. In this chapter we will consider a non-equilibrium scenario in which case the FDT no longer applies. Specifically, in order to treat heat radiation and transfer, regions of the system (usually separate objects) must be allowed to have different temperatures from each other. By assuming that the individual objects reach or are kept at a specific temperature in a much shorter timescale than reaching a global equilibrium, one can use the "equilibrium" noise in these objects, just with different temperatures.

This so-called local thermal equilibrium (LTE) approximation has been very successfully employed in the linear case $[52,51]$. We will see that this approach works very well in the nonlinear case as well and proceed to determine the non-equilibrium fluctuations in Section 4.1. With these fluctuations at hand, we continue by determining the effective electromagnetic potential or dielectric function in Section 4.2, just like for the equilibrium case in Section 3.2.

A general heat transfer formula is given in Appendix D, which will be applied to the case of a nanosphere in Section 4.3. The case of the plate is instructive to discuss Kirchhoff's law of radiation. The heat radiation of a nanosphere is interesting, because it is not necessary to consider the full space-dependent effective dielectric function. Rather, the effective properties can be taken into account as an effective polarizability. Since this is a single (frequency-dependent) number, we get a concise but explicit formula for the heat radiation, which allows us to discuss interesting qualitative properties of the heat radiation of nonlinear nanoparticles. An intriguing case we consider is the radiation of a purely nonlinear particle - we find that such a particle would always radiate energy away, even if it is colder than the environment. 


\subsection{Fluctuations in the local thermal equilibrium approximation}

\subsubsection{Non-equilibrium Rytov currents in the LTE approximation}

The LTE approximation is made by assuming that the statistics of noise will locally retain the form of Eq. 4.3, even if the temperature is not the same everywhere in space. This is physically best motivated, if the noise relaxes to (local) equilibrium at much faster time scales compared to the complete system. In particular, we will consider separated objects with different temperatures, but with every point inside any particular object at the same temperature. The assumption is therefore that these objects reach equilibrium within themselves much faster (for example through heat conduction) than it takes to transfer non-negligible amounts of energy between the different objects through the electromagnetic field (radiative heat transfer).

\section{Space-dependent temperature}

The equilibrium Rytov currents are given by Eq. (3.6), the noise-form of the FDT. Because the free Green's function is symmetric, it can be written as

$$
\left\langle\mathbf{F}_{\omega} \otimes \mathbf{F}_{\omega^{\prime}}^{*}\right\rangle^{\mathrm{eq}}=-\delta\left(\omega-\omega^{\prime}\right) b(\omega)\left(\operatorname{Im} \mathbb{G}_{0}^{-1}-\frac{1}{2 i} \tilde{\mathbb{V}}_{\mathrm{AH}}^{\mathrm{eq}}\right),
$$

where, as a reminder, the subscript "AH" denotes the anti-Hermitian part, $\mathbb{O}_{\mathrm{AH}}=\mathbb{O}-$ $\mathbb{O}^{\dagger}$. Temperature enters through the coefficient $b(\omega)$ [see Eq. (2.38)],

$$
b(\omega)=\frac{\hbar}{\pi \varepsilon_{0}} \frac{\omega^{2}}{c^{2}} \frac{1}{1-e^{-\frac{\hbar \omega}{k_{\mathrm{B}} T}}} .
$$

Unlike the correlator for fluctuations in Eq. (3.1), $\left\langle\mathbf{F}_{\omega} \otimes \mathbf{F}_{\omega^{\prime}}^{*}\right\rangle^{\text {eq }}$ is a local operator, depending actually on one spatial coordinate. This means the coefficient $b(\omega)$ in Eq. (4.1) really describes the temperature at the particular point $\mathbf{r}$. Letting the temperature be non-constant in space with $T \rightarrow T(\mathbf{r})$ and $b(\omega) \rightarrow b(\mathbf{r}, \omega)$, the "equilibrium" (constant) temperatures will be denoted as $T^{\mathrm{eq}}(\mathbf{r})=T$ and $b^{\mathrm{eq}}(\mathbf{r}, \omega)=b(\omega)$. The noise correlator can be written more explicitly as

$$
\begin{aligned}
\left\langle\mathbf{F}_{\omega}(\mathbf{r}) \otimes \mathbf{F}_{\omega^{\prime}}^{*}\left(\mathbf{r}^{\prime}\right)\right\rangle_{i j}^{\mathrm{eq}} & =-\delta\left(\omega-\omega^{\prime}\right) b^{\mathrm{eq}}(\mathbf{r}, \omega) \\
& \times\left[\operatorname{Im} \mathbb{G}_{0}^{-1}\left(\mathbf{r}, \mathbf{r}^{\prime}\right)_{i j}-\frac{1}{2 i}\left[\tilde{\varepsilon}_{i j}^{\mathrm{eq}}(\mathbf{r})\right]_{\mathrm{AH}} \delta\left(\mathbf{r}-\mathbf{r}^{\prime}\right)\right] .
\end{aligned}
$$


Where we remember that the first term in the brackets represents the infinitesimal vacuum dust [see Eq. (3.10)], which is non-zero only in the limit $\mathbf{r} \rightarrow \mathbf{r}^{\prime}$, while the second term gives noise in objects.

\section{LTE approximation for separated objects}

We follow the same procedure as in Section 3.2.2 and consider $N$ separated continuous objects (see Figure 3.2), giving us (see Eq. (3.24))

$$
\begin{aligned}
\chi^{(m)} & =\sum_{n=1}^{\infty} \chi_{n}^{(m)}, \\
\mathbb{V} & =\sum_{n=1}^{N} \mathbb{V}_{n} .
\end{aligned}
$$

As opposed to Section 3.2.2, however, the effective properties will not be considered to be determined in isolation; rather they are separated geometrically into pieces like the bare quantities. Thus the dielectric function $\tilde{\varepsilon}$ from Eq. (2.127) is written as

$$
\begin{aligned}
\tilde{\varepsilon} & =1+\sum_{n=1}^{N}\left(\tilde{\varepsilon}_{n}-1\right), \\
\tilde{\varepsilon}_{n} & =\varepsilon_{n}+N_{n}, \\
N_{n}(\mathbf{r}, \omega)_{i j} & =3 \int_{-\infty}^{\infty} \mathrm{d} \omega^{\prime} \chi_{n, i j k l}^{(3)}\left(\mathbf{r} ;-\omega, \omega, \omega^{\prime},-\omega^{\prime}\right)\left\langle\delta \mathbf{E}_{k}(\mathbf{r}) \otimes \delta \mathbf{E}_{l}(\mathbf{r})\right\rangle_{\omega^{\prime}} .
\end{aligned}
$$

The effective potential $\tilde{\mathbb{V}}$ is obtained from Eq. (2.125) or directly from the effective dielectric function above,

$$
\begin{aligned}
\tilde{\mathbb{V}} & =\sum_{n=1}^{N} \tilde{\mathbb{V}}_{n}, \\
\tilde{\mathbb{V}}_{n}\left(\mathbf{r}, \mathbf{r}^{\prime}\right)_{i j} & =\delta\left(\mathbf{r}-\mathbf{r}^{\prime}\right)\left[\tilde{\varepsilon}_{n}(\mathbf{r})-1\right] \\
& =\mathbb{V}_{n}+3 \mathcal{N}_{n}[\langle\delta \mathbf{E} \otimes \delta \mathbf{E}\rangle]
\end{aligned}
$$

Note again that, unlike for combination formulas in Section 3.2.2, the individual $\tilde{\varepsilon}_{n}$ and $\tilde{\mathbb{V}}_{n}$ do not correspond to the effective quantities of the object $n$ in the absence of other objects. This is purely a spatial separation.

Finally, we assign the temperature $T_{n}$ to each object and the temperature $T_{0}$ to vacuum, with the corresponding coefficients $b_{n}(\omega)$, as illustrated in Figure 4.1. The Rytov cur- 


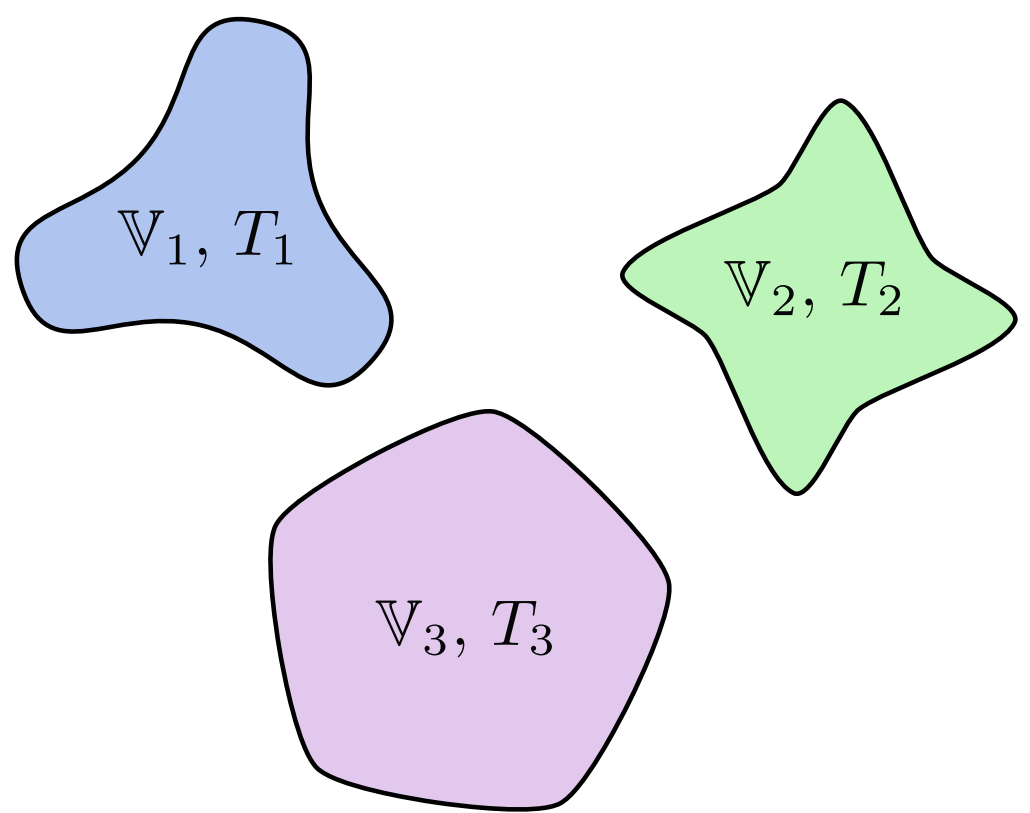

Figure 4.1.: Separation of the bare susceptibilities (potentials $\mathbb{V}_{n}$ ) between spatially separated objects with different temperatures $T_{n}$.

rents (noise) of Eq. (4.3) can now be written in the LTE approximation for separated objects,

$$
\left\langle\mathbf{F}_{\omega}(\mathbf{r}) \otimes \mathbf{F}_{\omega^{\prime}}^{*}\left(\mathbf{r}^{\prime}\right)\right\rangle^{\mathrm{LTE}}=\delta\left(\omega-\omega^{\prime}\right)\left[-b_{0}(\omega) \operatorname{Im} \mathbb{G}_{0}^{-1}\left(\mathbf{r}, \mathbf{r}^{\prime}\right)_{i j}+\sum_{n=1}^{N} \frac{b_{n}(\omega)}{2 i}\left(\tilde{\mathbb{V}}_{n}^{\mathrm{LTE}}\right)_{\mathrm{AH}}\right] .
$$

The superscript "LTE" means that all the averages in the effective quantities must be evaluated using the LTE noise.

By introducing a potential operator for the vacuum dust,

$$
\tilde{\mathbb{V}}_{0}^{\mathrm{LTE}}=\mathbb{V}_{0}=-\mathbb{G}_{0}^{-1},
$$

we can write the fluctuations even more briefly as

$$
\left\langle\mathbf{F}_{\omega}(\mathbf{r}) \otimes \mathbf{F}_{\omega^{\prime}}^{*}\left(\mathbf{r}^{\prime}\right)\right\rangle^{\mathrm{LTE}}=\delta\left(\omega-\omega^{\prime}\right) \sum_{n=0}^{N} \frac{b_{n}(\omega)}{2 i}\left(\tilde{\mathbb{V}}_{n}^{\mathrm{LTE}}\right)_{\mathrm{AH}}
$$

This is the final form for the noise correlator in the LTE approximation. 


\subsubsection{Non-equilibrium fluctuations in the LTE approximation}

\section{The fluctuations from non-equilibrium Rytov currents}

The system in the LTE is out of equilibrium, therefore we can not use the FDT [Eq. (2.36)] to obtain the fluctuations. Nevertheless, we remain in the limit of zero external field, so $\mathbf{E}_{\mathrm{in}}=\overline{\mathbf{E}}=0$. We can therefore use the same reasoning from the previous chapter, starting from Eq. (2.36), to obtain an analogue of Eq. 3.5,

$$
\left\langle\mathbf{F}_{\omega} \otimes \mathbf{F}_{\omega^{\prime}}^{*}\right\rangle^{\mathrm{LTE}}=\left(\mathbb{H}_{0}-\tilde{\mathbb{V}}^{\mathrm{LTE}}\right)_{\omega}\left\langle\delta \mathbf{E}_{\omega} \otimes \delta \mathbf{E}_{\omega^{\prime}}^{*}\right\rangle^{\mathrm{LTE}}\left(\mathbb{H}_{0}-\tilde{\mathbb{V}}^{\mathrm{LTE}}\right)_{\omega^{\prime}}^{\dagger} .
$$

The correlator for the fluctuations can be obtained by simply applying $\tilde{\mathbb{G}}^{\text {LTE }}=$ $\left(\mathbb{H}_{0}-\tilde{\mathbb{V}}^{\mathrm{LTE}}\right)^{-1}$ from the left and $\tilde{\mathbb{G}}^{\mathrm{LTE} \dagger}$ from the right,

$$
\left\langle\delta \mathbf{E}_{\omega} \otimes \delta \mathbf{E}_{\omega^{\prime}}^{*}\right\rangle^{\mathrm{LTE}}=\tilde{\mathbb{G}}^{\mathrm{LTE}}\left\langle\mathbf{F}_{\omega} \otimes \mathbf{F}_{\omega^{\prime}}^{*}\right\rangle^{\mathrm{LTE}} \tilde{\mathbb{G}}^{\mathrm{LTE} \dagger} .
$$

Using the noise correlator from Eq. (4.14), the above equation can be written as,

$$
\left\langle\delta \mathbf{E}_{\omega} \otimes \delta \mathbf{E}_{\omega^{\prime}}^{*}\right\rangle^{\mathrm{LTE}}=\delta\left(\omega-\omega^{\prime}\right) \sum_{n=0}^{N} \frac{b_{n}(\omega)}{2 i}\left(\tilde{\mathbb{G}} \tilde{\mathbb{V}}_{n} \tilde{\mathbb{G}}^{\dagger}\right)_{\mathrm{AH}}^{\mathrm{LTE}},
$$

where we used the easily provable identity $\mathbb{A B}_{\mathrm{AH}} \mathbb{A}^{\dagger}=\left(\mathbb{A B A}^{\dagger}\right)_{\mathrm{AH}}$. This is the final form for correlator of fluctuations in the LTE limit.

\section{Closing the system with a perturbative effective potential $\tilde{\mathbb{V}}_{n}^{\mathrm{LTE}}$}

As in the previous chapter, by keeping only leading order terms in $\chi^{(3)}$, the potentials $\tilde{\mathbb{V}}_{n}^{\mathrm{LTE}}$ defined by Eq. (4.11) can be written simply as

$$
\begin{aligned}
\tilde{\mathbb{V}}_{n}^{\mathrm{LTE}} & =\mathbb{V}_{n}+3 \mathcal{N}_{n}\left[\langle\delta \mathbf{E} \otimes \delta \mathbf{E}\rangle^{\mathrm{LTE}}\right] \\
\mathcal{N}_{n}\left[\langle\delta \mathbf{E} \otimes \delta \mathbf{E}\rangle^{\mathrm{LTE}}\right]\left(\mathbf{r}, \mathbf{r}^{\prime} ; \omega\right)_{i j} & =\delta\left(\mathbf{r}-\mathbf{r}^{\prime}\right) \int_{-\infty}^{\infty} \mathrm{d} \omega^{\prime} \chi_{n, i j k l}^{(3)}\left(\mathbf{r} ;-\omega, \omega, \omega^{\prime},-\omega^{\prime}\right) \\
& \times \sum_{n=0}^{N} b_{n}\left(\omega^{\prime}\right) \operatorname{Im}\left(\mathbb{G} \mathbb{V}_{n} \mathbb{G}^{\dagger}\right)\left(\mathbf{r}, \mathbf{r} ; \omega^{\prime}\right)_{k l}
\end{aligned}
$$

We will investigate the behavior of this quantity in the form of the effective dielectric function in more detail in the next section. 


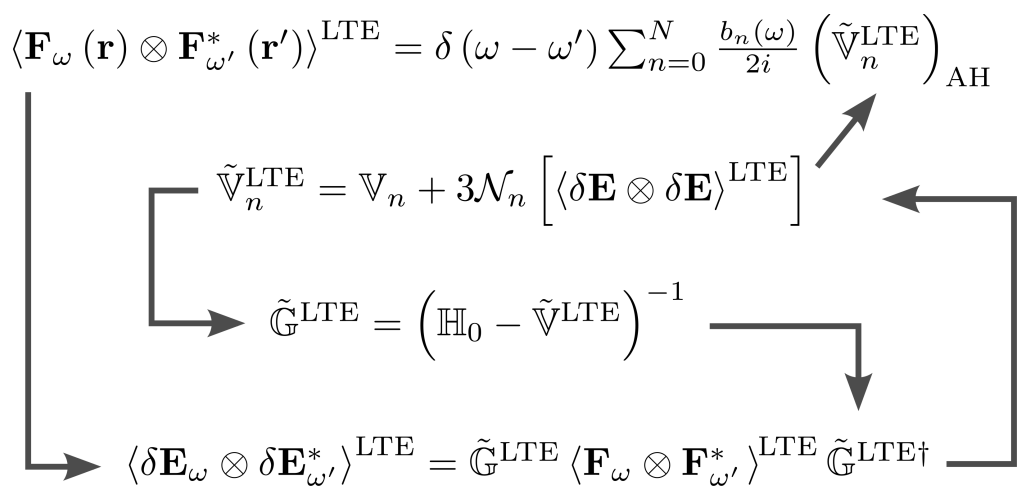

Figure 4.2.: Diagram showing the relations between equations for the non-equilibrium fluctuations with arrows corresponding to the "flow" of solution.

Figure 4.2 shows the interdependence between the various equations introduced in this chapter. In equilibrium the fluctuations are given by the FDT, and all other quantities, including the Rytov currents, follow from that. It is curious that in this case, however, the non-equilibrium Rytov currents were first fixed and only then the fluctuations, which no longer follow the FDT, could be determined.

It should also be noted that, just like in equilibrium (see Section 3.2.2), a choice needs to be made regarding which quantities remain constant under temperature change $\mathbb{V}_{n}$ or $\tilde{\mathbb{V}}_{n}^{\mathrm{LTE}}$. Eq. (4.18) gives only the difference. As before, we will use immutable (and homogeneous) bare susceptibilities $\chi^{(1)}$, whereas the effective quantities become temperature-dependent and inhomogeneous.

\subsection{The effective dielectric function out of thermal equilibrium}

\subsubsection{The effective dielectric function in the LTE approximation}

From Eq. (4.17) we see that the effects of the nonlinear susceptibility can be taken into account with an effective electromagnetic potential or dielectric function, just like in Chapter 3. Substituting the field correlator from Eq. (4.17) into Eq. (2.127), we can 
obtain a closed expression for the effective dielectric function,

$$
\begin{aligned}
\tilde{\varepsilon}_{i j}^{\mathrm{LTE}}(\mathbf{r}, \omega) & =\varepsilon_{i j}(\mathbf{r}, \omega)+N^{\mathrm{LTE}}(\mathbf{r}, \omega)_{i j}, \\
N^{\mathrm{LTE}}(\mathbf{r}, \omega)_{i j} & =3 \int_{-\infty}^{\infty} \mathrm{d} \omega^{\prime} \chi_{i j k l}^{(3)}\left(\mathbf{r} ;-\omega, \omega, \omega^{\prime},-\omega^{\prime}\right) \\
& \times \sum_{m=0}^{N} b_{m}\left(\omega^{\prime}\right) \operatorname{Im}\left(\mathbb{G} \mathbb{V}_{m} \mathbb{G}^{\dagger}\right)\left(\mathbf{r}, \mathbf{r} ; \omega^{\prime}\right)_{k l} .
\end{aligned}
$$

Notice that the equilibrium expression from Eq. (3.17) is recovered if all temperatures $T_{m}$ are equal since $\sum_{m=0}^{N} \operatorname{Im}\left(\mathbb{G} \mathbb{V}_{m} \mathbb{G}^{\dagger}\right)=\operatorname{Im} \tilde{\mathbb{G}}$.

As discussed in Section 3.2, Eq. (4.21) can be written equivalently as an integral over positive frequencies as well,

$$
\begin{aligned}
N^{\mathrm{LTE}}(\mathbf{r}, \omega)_{i j} & =3 \int_{0}^{\infty} \mathrm{d} \omega^{\prime} \chi_{i j k l}^{(3)}\left(\mathbf{r} ;-\omega, \omega, \omega^{\prime},-\omega^{\prime}\right) \\
& \times \sum_{m=0}^{N} a_{m}\left(\omega^{\prime}\right) \operatorname{Im}\left(\mathbb{G} \mathbb{V}_{m} \mathbb{G}^{\dagger}\right)\left(\mathbf{r}, \mathbf{r} ; \omega^{\prime}\right)_{k l}, \\
a_{m}(\omega) & =b_{m}(\omega)-b_{m}(-\omega) \\
& =\frac{\hbar}{\pi \varepsilon_{0}} \frac{\omega^{2}}{c^{2}} \operatorname{coth}\left(\frac{\hbar \omega}{2 k_{\mathrm{B}} T_{m}}\right) .
\end{aligned}
$$

\subsubsection{The non-equilibrium part to the effective dielectric function}

In order to distinguish and highlight the non-equilibrium properties of $\tilde{\mathbb{V}}^{\mathrm{LTE}}$ and $\tilde{\varepsilon}^{\mathrm{LTE}}$, it is useful to look at the difference of these quantities to their equilibrium counterparts, $\tilde{\mathbb{V}}^{\mathrm{eq}}$ and $\tilde{\varepsilon}^{\mathrm{eq}}$. We will denote this difference with the superscript " $\Delta T$ " to signify that it is the contribution due to temperature differences,

$$
\begin{aligned}
\mathbb{N}^{\Delta T}\left(\mathbf{r}, \mathbf{r}^{\prime} ; \omega\right)_{i j} & =\delta\left(\mathbf{r}-\mathbf{r}^{\prime}\right) N^{\Delta T}(\mathbf{r}, \omega)_{i j}, \\
\mathbb{N}^{\Delta T} & =\tilde{\mathbb{V}}^{\mathrm{LTE}}-\tilde{\mathbb{V}}^{\mathrm{eq}} \\
& =3 \mathcal{N}\left[\langle\delta \mathbf{E} \otimes \delta \mathbf{E}\rangle^{\mathrm{LTE}}-\langle\delta \mathbf{E} \otimes \delta \mathbf{E}\rangle^{\mathrm{eq}}\right], \\
N^{\Delta T} & =\tilde{\varepsilon}^{\mathrm{LTE}}-\tilde{\varepsilon}^{\mathrm{eq}} .
\end{aligned}
$$

The last expression can be evaluated directly using Eqs. (4.21) and (3.17). Practically, however, it is easier to use the expression for $\tilde{\varepsilon}^{\mathrm{LTE}}$ and set the temperatures equal. It is important, however, to choose a particular temperature for $\tilde{\varepsilon}^{\mathrm{eq}}$. Our choice is to consider 


\section{Thermal imbalance in nonlinear systems and radiative heat transfer}

the "reference temperature" to be the temperature of the object that the position vector $\mathbf{r}$ is in. That is, if $\mathbf{r} \in V_{n}$, then in $\tilde{\varepsilon}^{\text {eq }}$ we choose $b(\omega)=b_{n}(\omega)$. We therefore obtain the following expression,

$$
\begin{aligned}
N^{\Delta T}\left(\mathbf{r} \in V_{n}, \omega\right)_{i j} & =3 \int_{-\infty}^{\infty} \mathrm{d} \omega^{\prime} \chi_{i j k l}^{(3)}\left(\mathbf{r} ;-\omega, \omega, \omega^{\prime},-\omega^{\prime}\right) \\
& \times \sum_{m=0}^{N}\left[b_{m}\left(\omega^{\prime}\right)-b_{n}\left(\omega^{\prime}\right)\right] \operatorname{Im}\left(\mathbb{G} \mathbb{V}_{m} \mathbb{G}^{\dagger}\right)\left(\mathbf{r}, \mathbf{r} ; \omega^{\prime}\right)_{k l} .
\end{aligned}
$$

One can see now directly that $N^{\Delta T}$ becomes zero in object $n$ if the temperature of all other objects is the same.

We can also see from Eq. (4.27) that the effective dielectric function of a particular object starts to directly depend on the temperatures of other objects. While local temperaturedependence of epsilon is well known (for example, ordinary thermal expansion leads to changes in the dielectric function), the dependence on the temperature at other locations and even other objects is a novel prediction.

Furthermore, Eq. (4.27) is neat since it shows that really only objects which have different temperatures contribute, while the terms for objects $n$ and $m$ with $T_{n}=T_{m}$ will be zero. Most importantly, that includes the contribution from the same body $m=n$. That means in all non-zero terms of Eq. (4.27) the two Green's functions will only connect points in different objects, therefore avoiding the divergence $\operatorname{Im} \mathbb{G}(\mathbf{r}, \mathbf{r})$ as discussed in the previous chapter in Section 3.2. The divergence is still there, in $\tilde{\varepsilon}^{\mathrm{eq}}$, but we can proceed to study the physically relevant part $N^{\Delta T}$ without problems, exactly like the distance dependent part of the effective potential in Section 3.2.3.

\subsubsection{One object in vacuum - passive gain media}

A special case to consider is a single object at temperature $T_{\text {obj }}$ in vacuum at temperature $T_{\text {env }}$. In that case Eq. (4.27) can be written explicitly as

$$
\begin{aligned}
N_{\text {single }}^{\Delta T}(\mathbf{r}, \omega)_{i j} & =-18 \pi \int_{-\infty}^{\infty} \mathrm{d} \omega^{\prime} \frac{c}{\omega^{\prime}} \chi_{i j k l}^{(3)}\left(\mathbf{r} ;-\omega, \omega, \omega^{\prime},-\omega^{\prime}\right) \\
& \times \sum_{m=0}^{N}\left[b_{\text {env }}\left(\omega^{\prime}\right)-b_{\text {obj }}\left(\omega^{\prime}\right)\right] \frac{1}{V_{0}} \int_{V_{0}} \mathrm{~d} \mathbf{r}^{\prime} G_{k m}\left(\mathbf{r}, \mathbf{r}^{\prime} ; \omega^{\prime}\right) G_{l m}^{*}\left(\mathbf{r}, \mathbf{r}^{\prime} ; \omega^{\prime}\right) .
\end{aligned}
$$


where we used Eq. (3.8) for the environment dust. Remember that $V_{0}$ is the volume of all space except for (absorbing) objects. This volume is infinite, but the integral $\frac{1}{V_{0}} \int_{V_{0}} \mathrm{~d} \mathbf{r}^{\prime}$ is nevertheless finite.

As an illustration, the space and temperature dependence of $N^{\Delta T}$ for the case of a single planar interface with homogeneous (and temperature independent) bare coefficients is shown in Figures 4.3a and 4.3b. It shows clearly how the effective dielectric function depends on the temperature of external objects (in this case, the environment). As mentioned above, this is a purely nonlinear effect, absent for linear materials.

\section{Kirchhoff's law of radiation}

From Eq. (4.28) and also Figure 4.3b, we can make an interesting observation regarding the Kirchhoff's law of radiation. While this law applies strictly only in equilibrium, it is valid in the LTE approximation as well. However, care must be taken if nonlinear materials are present, since the effective dielectric properties depend on the temperatures of all the objects. For example, comparing the absorption in equilibrium and emissivity after lowering the temperature of the environment to zero will seemingly violate the Kirchhoff's law. All measurements must be performed at equal conditions (same temperatures and locations of objects).

\section{Passive gain media}

Eq. (4.28) is interesting, because even though it is hard to predict the sign of $N_{\text {single }}^{\Delta T}$ due to the integration and the unknown sign of $\chi^{(3)}$, it is clear that the $N_{\text {single }}^{\Delta T}$ changes sign if $T_{\text {env }}$ and $T_{\text {obj }}$ are switched (because $b(\omega)$ is monotonic in temperature). This has some very pertinent consequences for the imaginary part of $\tilde{\varepsilon}$, which is generally positive for passive media, which can only dissipate passing light but not amplify it. Materials with negative $\operatorname{Im} \tilde{\varepsilon}$ are called gain media and normally require some energy source in order to achieve this.

We can see from Eq. (4.28), however, that passive (meaning $\operatorname{Im} \varepsilon, \operatorname{Im} \tilde{\varepsilon}>0, \operatorname{Im} \chi^{(3)}<$ 0 ) but nonlinear materials can become gain media in some cases. Specifically, this could happen in scenario where for some frequencies and temperatures $\left|\operatorname{Im} \tilde{\varepsilon}^{\mathrm{eq}}(\omega)\right|<$ $\left|\operatorname{Im} N^{\Delta T}(\omega)\right|$. Since $\operatorname{Im} N^{\Delta T}(\omega)$ depends on the sign of $T_{\mathrm{env}}-T_{\mathrm{obj}}$, this must happen either when $T_{\text {env }}>T_{\text {obj }}$ or $T_{\text {env }}<T_{\text {obj. }}$. This change in sign will also have a further very interesting effect on the heat radiation, which we will discuss below. 


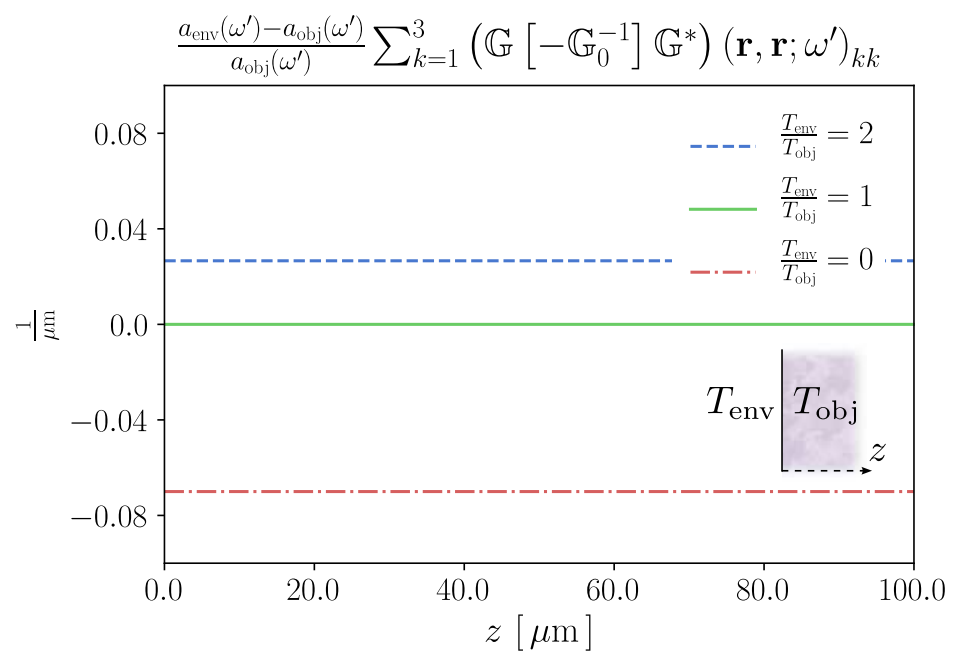

(a)

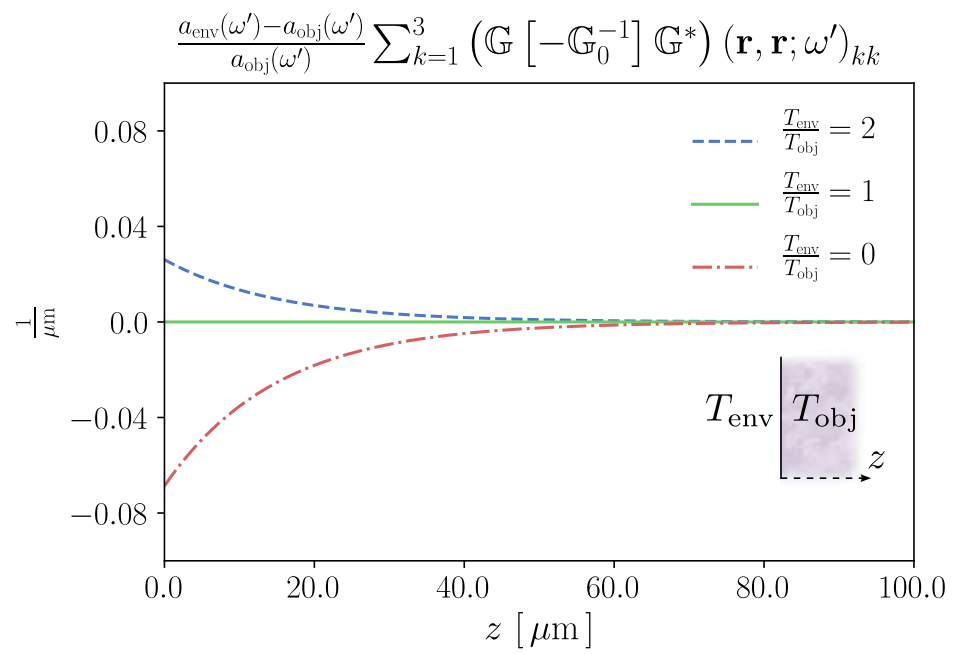

(b)

Figure 4.3.: Dependence of the effective dielectric function of an isotropic dielectric slab at a fixed temperature $T_{\mathrm{obj}}=300 \mathrm{~K}$ on the temperature $T_{\text {env }}$ of the environment, for the non-absorbing $\left[\varepsilon\left(\omega^{\prime}\right)=4\right.$, top] and absorbing $\left[\varepsilon\left(\omega^{\prime}\right)=4+i\right.$, bottom] case. These plots are calculated for $\omega^{\prime}=\frac{2 \pi c}{50 \mu \mathrm{m}}$, which sets the decay length into the material (skin depth). Compare with Figures 3.1 and 3.6. 


\subsection{Heat radiation and transfer in nonlinear systems}

In Appendix D a general heat transfer formula (D.5) is derived. Interestingly, it retains the same form for linear and nonlinear objects, as long as the correct effective dielectric properties are used.

\subsubsection{A single arbitrary object - anomalous modes}

Considering only a single object, Eq. (D.5) reduces to

$$
\begin{aligned}
H & =-\frac{1}{8 \pi \mu_{0}} \int_{-\infty}^{\infty} \mathrm{d} \omega \frac{1}{\omega}\left[b_{\mathrm{obj}}(\omega)-b_{\mathrm{env}}(\omega)\right] \\
& \times \operatorname{Tr}\left[(\tilde{\mathbb{V}})_{\mathrm{AH}} \tilde{\mathbb{G}}\left(\tilde{\mathbb{V}}_{0}\right)_{\mathrm{AH}} \tilde{\mathbb{G}}^{\dagger}\right] .
\end{aligned}
$$

If we make the rather significant simplification that the material is isotropic, $\tilde{\varepsilon}_{i j}=\delta_{i j} \tilde{\varepsilon}$ (see Section 2.2.1 for the conditions on $\chi^{(3)}$ ), then we can further reduce this to

$$
\begin{aligned}
H & =-\frac{1}{8 \pi \mu_{0}} \int_{-\infty}^{\infty} \mathrm{d} \omega \frac{1}{\omega}\left[b_{\text {obj }}(\omega)-b_{\text {env }}(\omega)\right] \\
& \times \frac{1}{V_{\text {env }}} \int_{\substack{\mathbf{r}_{1} \in V_{\text {obj }} \\
\mathbf{r}_{2} \in V_{\text {env }}}} \mathrm{d} \mathbf{r}_{1} \mathrm{~d} \mathbf{r}_{2} \operatorname{Im} \tilde{\varepsilon}\left(\mathbf{r}_{1}, \omega\right) \operatorname{Im} \tilde{\varepsilon}\left(\mathbf{r}_{2}, \omega\right) \sum_{i j}\left|\tilde{G}_{i j}\left(\mathbf{r}_{1}, \mathbf{r}_{2} ; \omega\right)\right|^{2} .
\end{aligned}
$$

In general, this expression involves many additional nonlinear terms due to the product of three effective quantities.

Most interesting, however, is the possibility of so-called anomalous modes or phases, where heat radiation is in the wrong direction. That is because, as we discussed below Eq. (4.28), the imaginary part of $\tilde{\varepsilon}$ can become negative for some combination of temperatures and positive if the temperatures are switched. Since Eq. (4.30) contains also the factor, $b_{\mathrm{obj}}(\omega)-b_{\mathrm{env}}(\omega)$, the direction of heat flow (for that particular frequency) remains unchanged, thus flowing in the wrong direction, from a cold body to the hot environment; hence the name "anomalous modes".

Reversing the heat direction of some frequency ranges is not necessarily a problem, if the total heat still flows from hot to cold regions. This can correspond, for example, to nonlinear processes which absorb in a wide range of frequencies but emit in a narrow range. Note that this is not possible in the linear case, because different frequencies are uncoupled. 


\subsubsection{Example a nonlinear nanosphere}

\section{The effective dielectric function}

A useful and simple example system for studying heat radiation is a single nanosphere in vacuum. In the limit where its radius is much smaller than the thermal wavelength $\lambda_{T}=\frac{\hbar c}{k_{\mathrm{B}} T}$ and the skin depth $\delta=\frac{1}{\operatorname{Im} \sqrt{\varepsilon \mu}} \frac{c}{\omega}$, the (linear) Green's function connecting points inside and outside the nanosphere can be approximated [51] as

$$
\mathbb{G}_{\text {sphere }}=\frac{3}{\varepsilon+2} \mathbb{G}_{0} .
$$

The thermal part of the effective dielectric function can then be almost directly calculated from Eq. 4.27. First, using $\mathbb{V}_{m}=-\mathbb{G}_{0}^{-1}$ (remembering to exclude the volume of the sphere), we have

$$
\begin{aligned}
N_{\text {sphere }}^{\Delta T}(\mathbf{r} ; \omega)_{i j} & =-3 \int_{-\infty}^{\infty} \mathrm{d} \omega^{\prime} \chi_{i j k l}^{(3)}\left(\mathbf{r} ;-\omega, \omega, \omega^{\prime},-\omega^{\prime}\right) \\
& \times\left[b_{\mathrm{env}}\left(\omega^{\prime}\right)-b_{\text {obj }}\left(\omega^{\prime}\right)\right]\left[\mathbb{G I m}\left(-\mathbb{G}_{0}^{-1}\right) \mathbb{G}^{\dagger}\right]\left(\mathbf{r}, \mathbf{r} ; \omega^{\prime}\right)_{k l} .
\end{aligned}
$$

The Green's functions in $\mathbb{G I m}\left(-\mathbb{G}_{0}^{-1}\right) \mathbb{G}^{\dagger}$ connect only points in the sphere with those outside, so we can use the approximation in Eq. (4.31). Since the volume of the sphere is negligible (meaning the properties are the same everywhere inside), we obtain simply

$$
\mathbb{G} \operatorname{Im}\left(-\mathbb{G}_{0}^{-1}\right) \mathbb{G}^{\dagger}=\left|\frac{3}{\varepsilon+2}\right|^{2} \operatorname{Im} \mathbb{G}_{0}
$$

Using Eq. (3.8), the thermal part of the effective dielectric function becomes

$$
\begin{aligned}
N_{\text {sphere }}^{\Delta T}(\omega)_{i j} & =-\frac{1}{2 \pi} \int_{-\infty}^{\infty} \mathrm{d} \omega^{\prime} \chi_{i j k k}^{(3)}\left(-\omega, \omega, \omega^{\prime},-\omega^{\prime}\right) \\
& \times \frac{\omega^{\prime}}{c}\left|\frac{3}{\varepsilon\left(\omega^{\prime}\right)+2}\right|^{2}\left[b_{\text {env }}\left(\omega^{\prime}\right)-b_{\text {obj }}\left(\omega^{\prime}\right)\right],
\end{aligned}
$$

which will be used to calculate the thermal contribution to the equilibrium polarizability below. 


\section{The effective polarizability and heat radiation}

The polarizability of a nanosphere is defined as

$$
\tilde{\alpha} \equiv \frac{\tilde{\varepsilon}-1}{\tilde{\varepsilon}+2} R^{3} .
$$

Using Eq. (4.34), we can determine the effective polarizability with respect to the equilibrium value,

$$
\tilde{\alpha}^{\mathrm{LTE}}=\tilde{\alpha}^{\mathrm{eq}}\left[1+\frac{3}{(\varepsilon-1)(\varepsilon+2)} N_{\text {sphere }}^{\Delta T}\right]
$$

The polarizability governs the radiation of small spheres [11],

$$
H=\frac{4 \varepsilon_{0}}{\pi^{2} c} \int_{-\infty}^{\infty} \mathrm{d} \omega \omega^{2}\left[b_{\text {obj }}(\omega)-b_{\text {env }}(\omega)\right] \operatorname{Im} \tilde{\alpha}^{\operatorname{LTE}}(\omega) .
$$

Since there is no problem with an inhomogeneous effective dielectric function in the case of a nanosphere, the known formula can be applied directly.

\section{The non-absorbing nanosphere}

As an intriguing example, we consider an extreme case - a nanosphere that is nonabsorbing in equilibrium,

$$
\operatorname{Im} \tilde{\varepsilon}^{\mathrm{eq}}=\operatorname{Im} \tilde{\alpha}^{\mathrm{eq}}=0 .
$$

By Eqs. (4.36) and (4.37), the sphere can only emit or absorb energy through a finite $\operatorname{Im} \chi^{(3)}$. Explicitly, the heat radiation is

$$
\begin{aligned}
H & =-54 \frac{\varepsilon_{0}}{\pi^{3} c^{3}} \int_{-\infty}^{\infty} \mathrm{d} \omega \int_{-\infty}^{\infty} \mathrm{d} \omega^{\prime} \omega^{2} \omega^{\prime} \operatorname{Im} \chi^{(3)}\left(-\omega, \omega, \omega^{\prime},-\omega^{\prime}\right) \\
& \times \frac{\left[b_{\mathrm{obj}}(\omega)-b_{\mathrm{env}}(\omega)\right]\left[b_{\mathrm{obj}}\left(\omega^{\prime}\right)-b_{\mathrm{env}}\left(\omega^{\prime}\right)\right]}{[\varepsilon(\omega)+2]^{2}\left[\varepsilon\left(\omega^{\prime}\right)+2\right]^{2}} .
\end{aligned}
$$

In this case, the heat radiation remains completely unchanged if the temperatures of the sphere and environment are interchanged. Since $\operatorname{Im} \chi^{(3)}<0$ in passive media [13], Eq. (4.39) yields $H>0$ for all temperatures, which would violate the laws of thermodynamics. 


\subsection{Summary}

By applying the LTE approximation on the results obtained in the previous chapter, we obtained the noise and fluctuations in a (nonlinear) system, where bodies can have different temperatures. This gave a yet another (generally inhomogeneous) contribution to the effective dielectric function.

Surprisingly, however, this contribution changes signs if the temperatures of two bodies were swapped. This paves way for the possibility of pseudo-gain media: otherwise passive media where waves of some frequency range experience gain instead of absorption due to "leeching" energy from fluctuations.

The results for radiative heat transfer were even more interesting, since these pseudogain regimes also tend to reverse the direction of heat flow (in a particular frequency range). This example was taken to the extreme with the radiation of a (linearly) nonabsorbing nanosphere: such an object would always radiate away more heat than absorb from the environment, even if it is at a lower temperature. 


\section{Nonlinear fluctuations in the presence of external fields}

Moving even further away from equilibrium, the goal of the this chapter is to investigate the influence of external fields on the fluctuations and Rytov currents. While in the linear case the fluctuations are decoupled from the incoming field due to the superposition principle, in nonlinear systems the fluctuations are expected to depend directly on the external field. We will find that, unlike in equilibrium, the FDT does not contain enough information to completely fix this dependence. Even more assumptions need to be made to predict non-equilibrium behavior.

After a short example of an exact calculation, the van der Waals force in a constant external field in Section 5.1, we turn our attention to the general case. Based on arbitrarily nonlinear scalar model in Section 5.2, we map the conditions that the noise has to follow in either the effective (with coefficients corresponding to response measurements) or bare (corresponding more to the model used in Chapters 3 and 4) equation of motion. While the general conditions are theoretically pleasing, they are better illustrated in a perturbative case with third order nonlinearities in Section 5.3, which corresponds also more closely with previous chapters. While the noise and fluctuations cannot be unambiguously determined, a useful framework is developed for testing different assumptions on noise. The non-equilibrium fluctuations for two specific cases are compared explicitly, which give two clearly different predictions that could be tested experimentally.

The chapter is concluded by bringing the story back to electromagnetic fluctuations in Section 5.4, where we use the simplest noise that still obeys the necessary conditions: a constant one. Nevertheless, a rich and explicit field dependence of the fluctuations is still obtained.

\subsection{Introductory example - van der Waals force in a constant field}

In Ref. [84], we studied a very particular system of nonlinear polarizable particles, connected through regular dipole-dipole interaction, in a static electric field. Since it is a 


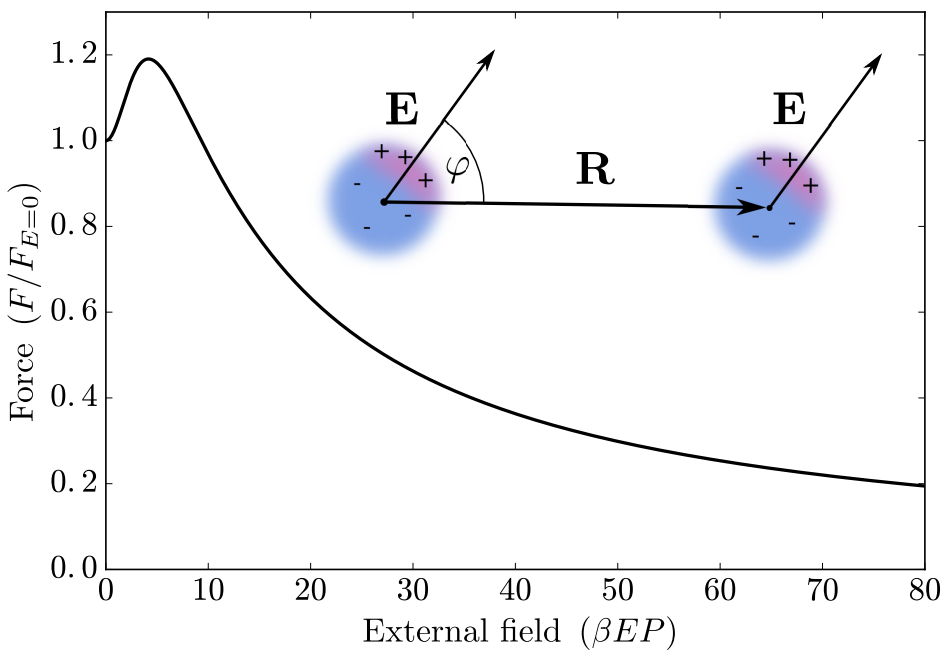

Figure 5.1.: Casimir force between two saturating particles in an external electric field E. The angle between the field and the vector $\mathbf{R}$ is $\varphi=\arccos \left(\frac{1}{\sqrt{3}}\right)$.

classical model with a fixed, microscopic Hamiltonian [14, 24, 26], all calculations could be carried out directly. There was no need to introduce noise by hand or use the FDT.

Compared to regular harmonic dipoles, able to respond in a limitless fashion to an electric field, these particles were given a hard limit to their maximum dipole moment in order to make them nonlinear (a bit like an opposite of a muffin tin potential). They behave like harmonic dipoles, if there is a small or no electric field, but within higher fields they become harder and harder to polarize - thus exhibiting a nonlinear response.

We were interested in the force between these particles. Under general conditions, putting polarizable particles in an electric field induces average dipole moments, which dominates the force between them at long distances. This deterministic force can be attractive or repulsive, however. By carefully choosing the direction of the electric field, as in Figure 5.1, this contribution could be canceled altogether.

Figure 5.1 shows that the force vanishes at very high fields. This is because the dominant contribution at high fields originates from the interaction of a strong induced dipole and a dipole with a negligible effective polarizability. If the dipole is strongly saturated, there is very little "room" for fluctuations. This effective polarizability would represent the effective dielectric function in the case of nonlinear fluctuational electrodynamics. It is the goal in this chapter to step closer to calculating the Casimir force and also heat transfer in the presence of strong external fields, by seeking to calculate the non-equilibrium fluctuations. 


\subsection{Stochastic scalar model - general approach}

In order to avoid some peculiarities of the Helmholtz equation, nonlinear fluctuations (or rather, fluctuations in nonlinear systems) will be investigated here in a simplified manner. We treat here a scalar model and ignore the complexities arising from differential operators. More generally, one would have to look at the problem in Fourier space (time derivatives) and/or employ a Green's function formalism (which was done for the EM).

\subsubsection{The phenomenological model}

We are interested in the fluctuating field or observable $x$ in a system which is being driven by an external deterministic source $a$. As opposed to the approach of Chapter 2, we do not yet fix the dynamics that this observable has to obey, but would rather aim to obtain this from measurements. The same goes for any fluctuations or noise sources.

An interesting aspect of fluctuating systems is that the observable $x$ by itself is not strictly speaking a "measurable quantity". The reason for this is that the values at different times are by definition random and therefore repeating the experiment can and will yield different results. Rather one must consider different kinds of averages like $\langle x\rangle$ and $\left\langle x^{2}\right\rangle$ to describe experiments. In equilibrium it is sufficient to average the values of an observable at different times. However, when the system is driven out of equilibrium (in this case by the external source $a$ ), the probability distribution of the fluctuating quantity becomes generally time-dependent. Therefore the averages must be considered to be ensemble averages or averages over many realizations of the fluctuating quantities (see Section 2.1.3).

By measuring $\langle x\rangle$ for different driving sources $a$, the effective response function can be obtained,

$$
\tilde{g}(a)=\langle x\rangle .
$$

This function could be linear or nonlinear, and would need to be determined for all possible values of $a$. Since that is not usually feasible experimentally, different kinds of models are used, such that only a small number of parameters would need to be determined. However, usually the model is given for the inverse $\tilde{h}$ of the response function ${ }^{1}$ (if it exists), such that

$$
\tilde{h}(\langle x\rangle)=a .
$$

\footnotetext{
${ }^{1}$ The model could be for example $\tilde{h}(v)=\left(\frac{d}{d t}+\gamma_{0}\right) v$ in the case of underdamped Brownian motion or $\tilde{h}(\mathbf{E})=\left(\nabla \times \nabla-\frac{\omega^{2}}{c^{2}}\right) \mathbf{E}$ for electrodynamics in vacuum. Note that these examples are linear in the field, which does not have to be the case.
} 


\section{Nonlinear fluctuations in the presence of external fields}

As in the previous chapters, the functions with tildes are called the "effective" response $(\tilde{g})$ and model $(\tilde{h})$ for the averaged quantity $\langle x\rangle$, in contrast to the "intrinsic" or bare response and model for the fluctuating quantity $x$ itself, which are as of yet unknown.

For the following, it is useful to represent Eqs. (5.1) and (5.1) as a power series,

$$
\begin{gathered}
\tilde{g}(a)=\sum_{m=0}^{\infty} \tilde{g}^{(m)} a^{m}, \\
\tilde{h}(\langle x\rangle)=\sum_{m=0}^{\infty} \tilde{h}^{(m)}\langle x\rangle^{m},
\end{gathered}
$$

which also more closely mimics the description of the nonlinear electromagnetic field as given in Section 2.2.

\subsubsection{The fluctuations and effective noise}

Knowing the effective response (5.1) or the model (5.2) determines the dynamics of $\langle x\rangle$ for any external source, which is useful if the fluctuations do not play a role. In cases where the fluctuations are absent $(\langle x\rangle=x)$, the knowledge of $\tilde{g}$ and $\tilde{h}$ is enough to fully describe the system. As we have seen already for the electromagnetic case, however, not only the mean of the observable $x$ but also the higher moments $\left\langle x^{n}\right\rangle$ are important, giving rise to the Casimir effect and heat radiation, for example. The response function itself does not give that information directly.

Fluctuations are defined as the deviation from the mean,

$$
\delta x=x-\langle x\rangle .
$$

By the above definition, fluctuations themselves must always have a zero mean, $\langle\delta x\rangle=$ 0 . The goal is to determine the higher moments $\left\langle\delta x^{n}\right\rangle$ (equivalently, the probability distribution). Then any moment of $x$ can be represented in terms of $\langle x\rangle$ (which we get from the response) and the moments of the fluctuations by straightforward binomial expansion,

$$
\left\langle x^{n}\right\rangle=\sum_{m=0}^{n}\left(\begin{array}{c}
m \\
n-m
\end{array}\right)\langle x\rangle^{n-m}\left\langle\delta x^{m}\right\rangle .
$$

Note that if the fluctuations are Gaussian, then only $\left\langle\delta x^{2}\right\rangle$ is needed, with higher moments given by Isserlis' theorem (see Section 2.3.4). This is often assumed if the system is linear, but is generally not true for nonlinear systems. 
We can add fluctuations directly to Eqs. (5.1) and (5.2) by substituting $\langle x\rangle \rightarrow\langle x\rangle+\delta x$,

$$
\begin{aligned}
\tilde{h}(x) & =a+\tilde{n}, \\
\tilde{g}(a+\tilde{n}) & =x,
\end{aligned}
$$

where stochastic source,

$$
\tilde{n}=\tilde{h}(\langle x\rangle+\delta x)-\tilde{h}(\langle x\rangle),
$$

appears. These sources, which we call the effective noise, are chosen such that they produce the correct field $x$ from Eq. (5.7) (that is, obeying the FDT and all other restrictions). A tilde is used to denote the noise that produces the correct field from the effective response and model, which governs the behavior of $\langle x\rangle$. This is necessary because we will see that there are cases where it is useful to absorb deterministic parts of the noise into the model due to physical considerations (e.g. Chapters 3 and 4).

An important thing to note is that even though we defined the noise source, no new information was included. The noise and fluctuations are at this point just two sides of the same quantity,

$$
\begin{aligned}
\delta x & =\sum_{n=0}^{\infty} \sum_{k=1}^{\infty} \tilde{g}^{(n+k)}\left(\begin{array}{c}
n+k \\
n
\end{array}\right) a^{n} \tilde{n}^{k}, \\
\tilde{n} & =\sum_{n=0}^{\infty} \sum_{k=1}^{\infty} \tilde{h}^{(n+k)}\left(\begin{array}{c}
n+k \\
n
\end{array}\right)\langle x\rangle^{n} \delta x^{k} .
\end{aligned}
$$

Knowing one, the other can be determined directly by above equations. This is particularly obvious, to the point where the terms are used interchangeably, if the response and model are linear. Then the above equations simplify significantly, giving

$$
\begin{aligned}
\delta x & =\tilde{g}^{(1)} \tilde{n}, \\
\tilde{n} & =\tilde{h}^{(1)} \delta x .
\end{aligned}
$$

This also shows that in the linear case the noise and fluctuations are independent of $a$ - the fluctuations are induced purely by noise (see also Section 2.1.3). By contrast, the fluctuations in nonlinear systems depend on the external source. Also note that the average effective noise must be zero for linear systems.

\subsubsection{The statistical properties of the effective noise}

The statistical properties (the probability distribution) of the fluctuations and noise are encoded into their moments $\left\langle\delta x^{m}\right\rangle$ and $\left\langle\tilde{n}^{m}\right\rangle$. If the stochastic variables are Gaussian, it 


\section{Nonlinear fluctuations in the presence of external fields}

is enough to know the second moments (see Section 2.3.4), but this cannot be assumed for nonlinear systems.

Since $\delta x$ and $\tilde{n}$ are directly related, conditions on one of them affects directly the other. So far, the only restriction is that the mean of the fluctuations is zero by definition, $\langle\delta x\rangle=0$. The corresponding condition for $\tilde{n}$ is obtained from Eq. (5.10),

$$
\langle\tilde{g}(a+\tilde{n})\rangle=\tilde{g}(a),
$$

This equation can also be interpreted as a condition that, for any particular input $a$, the added effective noise $\tilde{n}$ must leave output $\langle x\rangle$ unchanged. Notice that the mean $\langle\tilde{n}\rangle$ is still not known, since it depends on the higher moments of $\delta x$ through Eq. (5.11).

To see more clearly the free parameters that are left, the moments of noise can be written in a formal power series as

$$
\left\langle\tilde{n}^{m}\right\rangle=\sum_{k=0}^{\infty} \tilde{K}_{k}^{m} a^{k},
$$

with $\tilde{K}_{0}^{0}=1$ and $\tilde{K}_{k>0}^{0}=0$, because we must have $\left\langle\tilde{n}^{0}\right\rangle=1$. Combining this with Eq. (5.14) results in the following condition,

$$
\sum_{m=0}^{\infty} \tilde{g}^{(m)} \sum_{k=0}^{m}\left(\begin{array}{c}
m \\
k
\end{array}\right)\left\langle\tilde{n}^{k}\right\rangle a^{m-k}=\sum_{m=0}^{\infty} \tilde{g}^{(m)} a^{m} .
$$

Since this must be valid for any $a$, the following system of equations can be obtained:

$$
\tilde{g}^{(p)}=\tilde{g}^{(p)}+\sum_{m=0}^{p} \sum_{n=1}^{\infty} \tilde{g}^{(n+m)}\left(\begin{array}{c}
n+m \\
n
\end{array}\right) \tilde{K}_{p-m}^{n} .
$$

Therefore, for every $p \geq 0$,

$$
\sum_{m=0}^{p} \sum_{n=1}^{\infty} \tilde{g}^{(n+m)}\left(\begin{array}{c}
n+m \\
n
\end{array}\right) \tilde{K}_{p-m}^{n}=0 .
$$

Here we have explicitly a relation between the measured quantities $\tilde{g}^{(m)}$ (which are just numbers in the scalar model) and the unknown quantities, which are elements of the matrix $\tilde{K}$.

Noticing that the sum over $m$ goes to $p$, allures to a recursive relation. Indeed, Eq. (5.18) 
can be given in a very useful recursive form. For every $p \geq 1$, we have

$$
\begin{aligned}
\sum_{n=1}^{\infty} \tilde{g}^{(n)} \tilde{K}_{p}^{n} & =-\sum_{m=1}^{p} \sum_{n=1}^{\infty} \tilde{g}^{(n+m)}\left(\begin{array}{c}
n+m \\
n
\end{array}\right) \tilde{K}_{p-m}^{n} \\
& =-\sum_{m=0}^{p-1} \sum_{n=1}^{\infty}\left[\tilde{g}^{(n+p-m)}\left(\begin{array}{c}
n+p-m \\
n
\end{array}\right)\right] \tilde{K}_{m}^{n}
\end{aligned}
$$

The key takeaway is that the system of equations constitutes a necessary, but not a sufficient condition on noise. There are simply more unknowns in $\tilde{K}$ than there are equations. Furthermore, knowing the moments of noise (of which the FDT fixes the second moment) in equilibrium determines only one row of $\tilde{K}$,

$$
\left\langle\tilde{n}^{m}\right\rangle^{\mathrm{eq}}=\tilde{K}_{0}^{m} .
$$

This means that even knowing everything in equilibrium, as well as Eq. (5.17), is not enough to fully determine the noise moments (and thus fluctuations) out of equilibrium. This is separate from the issue of distinction between the bare and effective quantities outlined in Section 2.3, which shall be investigated next.

\subsubsection{Zero-mean noise: the bare model}

\section{The relation between bare and effective models}

The average of the effective noise $\tilde{n}$ is generally nonzero by Eqs. (5.7) and (5.11),

$$
\begin{aligned}
\langle\tilde{n}\rangle & =\langle\tilde{h}(x)\rangle-\tilde{h}(\langle x\rangle) \\
& =\sum_{n=0}^{\infty} \sum_{k=1}^{\infty} \tilde{h}^{(n+k)}\left(\begin{array}{c}
n+k \\
n
\end{array}\right)\langle x\rangle^{n}\left\langle\delta x^{k}\right\rangle .
\end{aligned}
$$

As an average, $\langle\tilde{n}\rangle$ is not itself stochastic (fluctuating). As we saw in Chapters 2 to 4 , however, it was useful to consider the noise to have a zero mean and include this deterministic part implicitly in the so-called bare susceptibilities $\chi^{(n)}$. Using similar notation and terminology, we can define the following "bare" model as an alternative to Eq. (5.7),

$$
\begin{aligned}
h(x) & =a+n, \\
g(a+n) & =x, \\
\langle n\rangle & =0,
\end{aligned}
$$


Eq. (5.22) is the equivalent to the stochastic nonlinear Helmholtz equation, Eq. (2.116), where the new coefficients are related to the effective ones via

$$
\begin{aligned}
\langle h(x)\rangle & =a=\tilde{h}(\langle x\rangle), \\
0 & =\sum_{n=0}^{\infty}\langle x\rangle^{n}\left[\tilde{h}^{(n)}-\sum_{k=0}^{\infty} h^{(n+k)}\left(\begin{array}{c}
n+k \\
n
\end{array}\right)\left\langle\delta x^{k}\right\rangle\right] .
\end{aligned}
$$

The expression in the square brackets is in general not zero for each $n$, however, because the moments $\left\langle\delta x^{k}\right\rangle$ can also depend on $\langle x\rangle$. Making a similar expansion to Eq. (5.15), we write

$$
\left\langle\delta x^{k}\right\rangle=\sum_{m=0}^{\infty} D_{m}^{k}\langle x\rangle^{m},
$$

where $D_{0}^{0}=1, D_{k>0}^{0}=0, D_{k}^{1}=0$. The previous condition can then be written explicitly as

$$
\begin{aligned}
\tilde{h}^{(p)} & =\sum_{n=0}^{p} \sum_{k=0}^{\infty} h^{(n+k)}\left(\begin{array}{c}
n+k \\
n
\end{array}\right) D_{p-n}^{k} \\
& =h^{(p)}+\sum_{n=0}^{p} \sum_{k=2}^{\infty} h^{(n+k)}\left(\begin{array}{c}
n+k \\
n
\end{array}\right) D_{p-n}^{k} .
\end{aligned}
$$

In general, the relation between the effective and bare equations depends on how the moments $\left\langle\delta x^{k}\right\rangle$ depend on $\langle x\rangle$ or, equivalently, $a$.

\section{The pseudo-effective model}

From Eq. (5.26), one can formally obtain the effective coefficients as

$$
\begin{aligned}
\tilde{h}^{\prime(p)} & =\sum_{k=0}^{\infty} h^{(p+k)}\left(\begin{array}{c}
p+k \\
p
\end{array}\right)\left\langle\delta x^{k}\right\rangle \\
& =\sum_{k=0}^{\infty} h^{(p+k)}\left(\begin{array}{c}
p+k \\
p
\end{array}\right) \sum_{n=0}^{\infty} D_{n}^{k}\langle x\rangle^{n},
\end{aligned}
$$


where the coefficients $\tilde{h}^{\prime(n)}$ are themselves understood to be be dependent on $\langle x\rangle$. The first few coefficients are

$$
\begin{aligned}
\tilde{h}^{(0)} & =\sum_{k=0}^{\infty} h^{(k)}\left\langle\delta x^{k}\right\rangle \\
& =h^{(0)}+h^{(2)}\left\langle\delta x^{2}\right\rangle+\ldots, \\
\tilde{h}^{(1)} & =\sum_{k=0}^{\infty} h^{(k+1)}(k+1)\left\langle\delta x^{k}\right\rangle \\
& =h^{(1)}+3 h^{(3)}\left\langle\delta x^{2}\right\rangle+\ldots
\end{aligned}
$$

These are already familiar from Section 2.4.

Note that the response function itself remains correct, but only if it is applied to the mean,

$$
\begin{aligned}
\tilde{h}^{\prime}(y) & =\sum_{n=0}^{\infty} \tilde{h}^{\prime(n)} y^{n}=\sum_{n=0}^{\infty}\left[\sum_{k=0}^{\infty} h^{(n+k)}\left(\begin{array}{c}
n+k \\
n
\end{array}\right)\left\langle\delta x^{k}\right\rangle_{\langle x\rangle=y} y^{n}\right] \\
& =\sum_{n=0}^{\infty} \tilde{h}^{(n)} y^{n}=\tilde{h}(y) .
\end{aligned}
$$

This is just a different representation of $\tilde{h}$, where it is important to keep in mind that the primed coefficients depend on $\langle x\rangle$. For example, while $\tilde{h}^{(1)}$ represents the full linear response, the primed $\tilde{h}^{\prime(1)}$ in general does not, since the lower orders can include linear terms in $\langle x\rangle$ (such as $h^{(2)}\left\langle\delta x^{2}\right\rangle$ in $\tilde{h}^{\prime(0)}$ above).

\section{The bare noise $n$}

Using Eq. (5.22), the bare noise $n$ can be written as

$$
n=n-\langle n\rangle=h(x)-\langle h(x)\rangle,
$$

which in series form is

$$
n=\sum_{n=0}^{\infty}\langle x\rangle^{n} \sum_{k=0}^{\infty} h^{(n+k)}\left(\begin{array}{c}
n+k \\
n
\end{array}\right)\left[\delta x^{k}-\left\langle\delta x^{k}\right\rangle\right] .
$$

And similar to Eq. (5.10), there exists a dual relation for the fluctuations

$$
\delta x=\sum_{n=0}^{\infty} \sum_{k=0}^{\infty} g^{(n+k)}\left(\begin{array}{c}
n+k \\
n
\end{array}\right) a^{n}\left[n^{k}-\left\langle n^{k}\right\rangle\right] .
$$


Writing it this way, the condition $\langle\delta x\rangle=0$ is trivially true. However, it does give an alternative to Eq. (5.28).

Introducing a power series for the moments of $n$ as as an analogue to Eq. (5.15),

$$
\left\langle n^{m}\right\rangle=\sum_{k=0}^{\infty} K_{k}^{m} a^{k}
$$

where $K_{0}^{0}=1, K_{k>0}^{0}=0, K_{k}^{1}=0$, the condition $\langle g(a+n)\rangle=\langle x\rangle=\tilde{g}(a)$ can be written as

$$
\begin{aligned}
\sum_{p=0}^{\infty} \tilde{g}^{(p)} a^{p} & =\sum_{n=0}^{\infty} \sum_{k=0}^{\infty} g^{(n+k)}\left(\begin{array}{c}
n+k \\
n
\end{array}\right) a^{n}\left\langle n^{k}\right\rangle, \\
\tilde{g}^{(p)} & =g^{(n)}+\sum_{n=0}^{\infty} \sum_{k=2}^{\infty} g^{(n+k)}\left(\begin{array}{c}
n+k \\
n
\end{array}\right) K_{p-n}^{k} .
\end{aligned}
$$

Which is the mirror relation to Eq. (5.28).

\subsubsection{Summary and discussion about assumptions}

\section{The three models}

These models all give different descriptions of the same system:

1. By measuring the average $\langle x\rangle$ of a stochastic variable for various inputs $a$, a phenomenological model $\tilde{g}$ is built with an inverse $\tilde{h}$ [Eqs. (5.2) and (5.1)],

$$
\begin{aligned}
\tilde{h}(\langle x\rangle) & =a, \\
\tilde{g}(a) & =\langle x\rangle .
\end{aligned}
$$

2. By adding $\delta x=x-\langle x\rangle$ to the phenomenological equations the stochastic effective equations are obtained [Eqs (5.7) and (5.8)],

$$
\begin{aligned}
\tilde{h}(x) & =a+\tilde{n}, \\
\tilde{g}(a+\tilde{n}) & =x .
\end{aligned}
$$

3. Since $\langle\tilde{n}\rangle \neq 0$, a further bare model [Eqs (5.22) and (5.23)] can be postulated,

$$
\begin{aligned}
h(x) & =a+n, \\
g(a+n) & =x, \\
\langle n\rangle & =0 .
\end{aligned}
$$


Note that it is just as easy to start from the bare equations with $\langle n\rangle=0$ and obtain the stochastic and non-stochastic effective models, which is the route selected in Chapter 2.

\section{Translation between the models}

The difference between the effective and bare models is given by Eqs. (5.28) and (5.38),

$$
\begin{aligned}
& \tilde{h}^{(p)}=h^{(p)}+\sum_{n=0}^{p} \sum_{k=2}^{\infty} h^{(n+k)}\left(\begin{array}{c}
n+k \\
n
\end{array}\right) D_{p-n}^{k}, \\
& \tilde{g}^{(p)}=g^{(p)}+\sum_{n=0}^{\infty} \sum_{k=2}^{\infty} g^{(n+k)}\left(\begin{array}{c}
n+k \\
n
\end{array}\right) K_{p-n}^{k},
\end{aligned}
$$

where the non-equilibrium properties of the noise and fluctuations can be represented generally as [Eqs. (5.36) and (5.27)]

$$
\begin{aligned}
\left\langle\delta x^{k}\right\rangle & =\sum_{m=0}^{\infty} D_{m}^{k}\langle x\rangle^{m}, \\
\left\langle n^{m}\right\rangle & =\sum_{k=0}^{\infty} K_{k}^{m} a^{k} .
\end{aligned}
$$

An important point is that while for every bare model $h(x)$ there corresponds in principle an effective model $\tilde{h}(\langle x\rangle)$, then the reverse is not necessarily true.

\section{Noise, fluctuations, and assumptions}

The fluctuations and noise can be written formally in terms of each other,

$$
\begin{aligned}
\delta x & =\sum_{n=0}^{\infty} \sum_{k=1}^{\infty} \tilde{g}^{(n+k)}\left(\begin{array}{c}
n+k \\
n
\end{array}\right) a^{n} \tilde{n}^{k}, \\
\tilde{n} & =\sum_{n=0}^{\infty} \sum_{k=1}^{\infty} \tilde{h}^{(n+k)}\left(\begin{array}{c}
n+k \\
n
\end{array}\right)\langle x\rangle^{n} \delta x^{k}, \\
\delta x & =\sum_{n=0}^{\infty} \sum_{k=0}^{\infty} g^{(n+k)}\left(\begin{array}{c}
n+k \\
n
\end{array}\right) a^{n}\left[n^{k}-\left\langle n^{k}\right\rangle\right], \\
n & =\sum_{n=0}^{\infty} \sum_{k=0}^{\infty} h^{(n+k)}\left(\begin{array}{c}
n+k \\
n
\end{array}\right)\langle x\rangle^{n}\left[\delta x^{k}-\left\langle\delta x^{k}\right\rangle\right] .
\end{aligned}
$$


They are, however, unknown in general, besides conditions stemming from their definitions $(\langle\delta x\rangle=\langle x-\langle x\rangle\rangle=0,\langle n\rangle=0)$ :

$$
\begin{aligned}
\langle\delta x\rangle & =0, \\
\langle n\rangle & =0, \\
\langle\tilde{g}(a+\tilde{n})\rangle & =\tilde{g}(a), \\
\langle g(a+n)\rangle & =\tilde{g}(a) .
\end{aligned}
$$

The last two equations [for series forms see Eqs. (5.18) and (5.19)] are a necessary, but not a sufficient condition to determine either $n$ or $\tilde{n}$, even if the moments in equilibrium are known (the coefficients $\left.K_{0}^{n}, \tilde{K}_{0}^{n}\right)$. Higher moments $\left\langle\delta x^{m}\right\rangle$, which can be expressed in terms of these by Eqs. (5.50) and (5.52), are necessary to unambiguously describe the stochastic phenomena in the presence of a strong external field.

Therefore, in applying these equations, either additional information needs to be obtained or reasonable assumptions need to be made. These fall into two separate categories:

\section{Reactive noise or reactive effective properties?}

This is mostly a physical problem. Eq. (5.38) gives the difference between the effective and bare properties, but does not say if $h$ or $\tilde{h}$ should depend on fluctuations. The question is, which stochastic model corresponds to the underlying microscopic theory? In the context of EM field, it is the choice of whether the bare or effective properties remain unchanged if parts of the system are investigated in isolation (see Section 3.2.2). Since this choice affects physical properties, either case could be ruled out by experiment.

\section{What happens to noise out of equilibrium?}

This is a mathematical problem: fixing either $\delta x, n$, or $\tilde{n}$ in equilibrium (for example with the FDT) does not reduce the degrees of freedom enough to make definitive predictions out of equilibrium. The options include using a particular model for the $a$-dependence of the noise (the route taken in Refs. $[43,44]$ ) or use the noise from equilibrium (either $n=n^{\mathrm{eq}}$ or $\tilde{n}=\tilde{n}^{\mathrm{eq}}$ ) to approximate out of equilibrium fluctuations (which must depend on the external field through Eqs. (5.50) and (5.52)).

Nevertheless, some cases can be ruled out purely by Eqs. (5.56) and (5.57). For example Gaussian noise can be ruled out already in the perturbative analysis below. 


\subsection{Stochastic scalar model - perturbative approach}

The general case above is useful for general discussion and gives a good insight into what can be calculated and what assumptions must be made. However, more intuitive understanding can be obtained through explicit calculation in simplified cases.

\subsubsection{Perturbative models}

\section{The truncated response}

Mirroring Section 2.4, we limit the response to third order nonlinear terms,

$$
\begin{aligned}
& \tilde{h}(x)=\left(h_{0}-\tilde{\chi}^{(1)}\right) x-\tilde{\chi}^{(3)} x^{3}, \\
& h(x)=\left(h_{0}-\chi^{(1)}\right) x-\chi^{(3)} x^{3},
\end{aligned}
$$

and give leading order results. Here $h_{0}$ mimics the free Helmholtz operator $\mathbb{H}_{0}$, and $\tilde{\chi}^{(1)}, \tilde{\chi}^{(3)}$ the effective susceptibilities. As per the program, all calculations are given to leading order in $\chi^{(3)}$ (we anticipate that the effective $\tilde{\chi}^{(3)}$ and bare $\chi^{(3)}$ are equal). This means the inverses of the above equations can be easily found as,

$$
\begin{aligned}
& \tilde{g}(a)=\left(h_{0}-\tilde{\chi}^{(1)}\right)^{-1} a+\tilde{\chi}^{(3)}\left(h_{0}-\tilde{\chi}^{(1)}\right)^{-4} a^{3}, \\
& g(a)=\left(h_{0}-\chi^{(1)}\right)^{-1} a+\chi^{(3)}\left(h_{0}-\chi^{(1)}\right)^{-4} a^{3} .
\end{aligned}
$$

\section{The three models}

With explicit forms for the functions $\tilde{h}(x), h(x), \tilde{g}(a)$, and $g(a)$, the three models from the previous section can be written as follows:

1. The phenomenological model [Eqs. (5.2) and (5.1)],

$$
\begin{aligned}
\left(h_{0}-\tilde{\chi}^{(1)}\right)\langle x\rangle-\tilde{\chi}^{(3)}\langle x\rangle^{3} & =a, \\
\left(h_{0}-\tilde{\chi}^{(1)}\right)^{-1} a+\chi^{(3)}\left(h_{0}-\tilde{\chi}^{(1)}\right)^{-4} a^{3} & =\langle x\rangle .
\end{aligned}
$$

Notice that this supports three "equilibrium" $(a \rightarrow 0)$ solutions: the trivial $\langle x\rangle^{\text {eq }}=0$, but also $\pm \sqrt{\frac{\chi^{(3)}}{h_{0}-\tilde{\chi}^{(1)}}}$. We choose the solution where the system relaxes to zero with $\langle x\rangle^{\mathrm{eq}}=0$. Otherwise a zeroth order term would also need to be included in Eqs. (5.60) and (5.61). 
2. The effective model [Eqs (5.7) and (5.8)],

$$
\begin{aligned}
\left(h_{0}-\tilde{\chi}^{(1)}\right) x-\tilde{\chi}^{(3)} x^{3} & =a+\tilde{n}, \\
\left(h_{0}-\tilde{\chi}^{(1)}\right)^{-1}(a+\tilde{n})+\tilde{\chi}^{(3)}\left(h_{0}-\tilde{\chi}^{(1)}\right)^{-4}(a+\tilde{n})^{3} & =x .
\end{aligned}
$$

3. The bare model [Eqs (5.22) and (5.23)]

$$
\begin{aligned}
\left(h_{0}-\chi^{(1)}\right) x-\chi^{(3)} x^{3} & =a+n, \\
\left(h_{0}-\chi^{(1)}\right)^{-1}(a+n)+\chi^{(3)}\left(h_{0}-\chi^{(1)}\right)^{-4}(a+n)^{3} & =x, \\
\langle n\rangle & =0 .
\end{aligned}
$$

These equations must all hold true at the same time, but are useful in different contexts. The phenomenological model is used to relate the theory to linear and nonlinear response measurements. The effective or bare model are each useful depending on which is considered more "fundamental". For example, in the previous chapters we considered the bare coefficients to be immutable, which leads to a change in the effective coefficients.

\section{Translation equations}

The difference between the effective and bare models can be obtained directly from Eqs. (5.28) and (5.38),

$$
\begin{aligned}
& \tilde{h}^{(1)}=h^{(1)}+3 h^{(3)} D_{0}^{2}+h^{(3)} D_{1}^{3}, \\
& \tilde{h}^{(3)}=h^{(3)}+3 h^{(3)} D_{2}^{2}+h^{(3)} D_{3}^{3}, \\
& \tilde{g}^{(1)}=g^{(1)}+3 g^{(3)} K_{0}^{2}+g^{(3)} K_{1}^{3}, \\
& \tilde{g}^{(3)}=g^{(3)}+3 g^{(3)} K_{2}^{2}+g^{(3)} K_{3}^{3} .
\end{aligned}
$$

Now we keep only leading order terms in $\chi^{(3)}$. In that case the fluctuations and noise can be assumed to be Gaussian in linear order, meaning $\left\langle\delta x^{2}\right\rangle=\left\langle\delta x^{2}\right\rangle_{\langle x\rangle=0}+\mathcal{O}\left(\chi^{(3)}\right)$ and $\left\langle\delta x^{3}\right\rangle=\mathcal{O}\left(\chi^{(3)}\right)$. With this approximation, the above equations simplify to

$$
\begin{aligned}
& \tilde{\chi}^{(1)}=\chi^{(1)}+3 \chi^{(3)}\left\langle\delta x^{2}\right\rangle_{\langle x\rangle=0}, \\
& \tilde{\chi}^{(3)}=\chi^{(3)}, \\
& \tilde{g}^{(1)}=g^{(1)}+3 g^{(3)}\left\langle n^{2}\right\rangle_{a=0}, \\
& \tilde{g}^{(3)}=g^{(3)} .
\end{aligned}
$$


Since the effective and bare third order coefficients are equal, the tildes can be dropped from them. For the linear order, however, we keep them separated, because only the difference is known.

\subsubsection{Perturbative noise and fluctuations}

The noise and fluctuations are given by Eqs. (5.50) to (5.53),

$$
\begin{aligned}
\delta x & =\left(\tilde{g}^{(1)}+3 \tilde{g}^{(3)} a^{2}\right) \tilde{n}+3 g^{(3)} a \tilde{n}^{2}+g^{(3)} \tilde{n}^{3}, \\
\tilde{n} & =\left(\tilde{h}^{(1)}+3 h^{(3)}\langle x\rangle^{2}\right) \delta x+3 h^{(3)}\langle x\rangle \delta x^{2}+h^{(3)} \delta x^{3}, \\
\delta x & =\left(g^{(1)}+3 g^{(3)} a^{2}\right) n+3 g^{(3)} a\left[n^{2}-\left\langle n^{2}\right\rangle\right]+g^{(3)}\left[n^{3}-\left\langle n^{3}\right\rangle\right], \\
n & =\left(h^{(1)}+3 h^{(3)}\langle x\rangle^{2}\right) \delta x+3 h^{(3)}\langle x\rangle\left[\delta x^{2}-\left\langle\delta x^{2}\right\rangle\right]+h^{(3)}\left[\delta x^{3}-\left\langle\delta x^{3}\right\rangle\right] .
\end{aligned}
$$

These quantities are, however, unknown in general.

\section{Relation between effective and bare noise}

Subtracting the effective model, Eq. (5.64), from the bare model, Eq. (5.66), we also obtain the relation between the bare and effective noise,

$$
\begin{aligned}
n & =\tilde{n}+3 \chi^{(3)}\left\langle\delta x^{2}\right\rangle x, \\
& =\tilde{n}+\chi^{(3)} \frac{3 a\left\langle\tilde{n}^{2}\right\rangle+3\left\langle\tilde{n}^{2}\right\rangle \tilde{n}}{\left(h_{0}-\tilde{\chi}^{(1)}\right)^{3}} .
\end{aligned}
$$

The physical significance is that, as with the effective susceptibilities $\tilde{\chi}^{(1)}$ and $\chi^{(1)}$, we only know the difference between $\tilde{n}$ and $n$, since in the nonlinear term either one could be used (the change would be of order $\left(\chi^{(3)}\right)^{2}$ ). Fixing one of them, for example by some microscopic motivation, completely determines the other. Furthermore, we can see that both of them cannot be independent of the external source $a$ at the same time.

This equation has a couple of interesting consequences. First, the average of $\tilde{n}$ can be obtained easily,

$$
\langle\tilde{n}\rangle=-3 \chi^{(3)}\left\langle\delta x^{2}\right\rangle_{\langle x\rangle=0}\langle x\rangle .
$$

Secondly, the zero-mean part of the effective noise, $\delta \tilde{n}=\tilde{n}-\langle\tilde{n}\rangle$, is not equal to the bare noise,

$$
n=\delta \tilde{n}+3 \chi^{(3)}\left\langle\delta x^{2}\right\rangle_{\langle x\rangle=0} \delta x .
$$


5. Nonlinear fluctuations in the presence of external fields

This is shows that it was prudent not to take the tempting path of forcing the bare model as

$$
h^{\prime}(x)=\tilde{h}(x)-\langle\tilde{n}\rangle=a+\delta \tilde{n},
$$

because the bare noise $n$ is different from $\delta \tilde{n}$.

\section{Condition on effective noise}

As we saw in the general case, one of the few pieces of information regarding the noise is contained in the fact that $\langle\delta x\rangle=0$. The general conditions, Eqs. (5.18) and (5.19), can be written in the perturbative case, taking into account that only $\tilde{g}^{(1)}$ and $\tilde{g}^{(3)}$ are nonzero, as

$$
\tilde{g}^{(1)} \tilde{K}_{p}^{1}+\tilde{g}^{(3)} \tilde{K}_{p}^{3}=-3 \tilde{g}^{(3)}\left(\tilde{K}_{p-1}^{2}+\tilde{K}_{p-2}^{1}\right)
$$

where any $\tilde{K}_{m<0}^{n}=0$. Taking into account that $\tilde{K}_{n}^{1}, \tilde{K}_{n}^{3}=\mathcal{O}\left(\chi^{(3)}\right)$, this expression shortens to

$$
\tilde{g}^{(1)} \tilde{K}_{p}^{1}=-3 \tilde{g}^{(3)} \tilde{K}_{p-1}^{2} .
$$

As an example, the first few terms would be

$$
\begin{aligned}
& \tilde{g}^{(1)} \tilde{K}_{0}^{1}=0, \\
& \tilde{g}^{(1)} \tilde{K}_{1}^{1}=3 \tilde{g}^{(3)} \tilde{K}_{0}^{2}, \\
& \tilde{g}^{(1)} \tilde{K}_{2}^{1}=3 \tilde{g}^{(3)} \tilde{K}_{1}^{2} .
\end{aligned}
$$

It can be seen that these expressions relate $\langle\tilde{n}\rangle$ to $\left\langle\tilde{n}^{2}\right\rangle$,

$$
\tilde{g}^{(1)}\langle\tilde{n}\rangle=3 \tilde{g}^{(3)}\left\langle\tilde{n}^{2}\right\rangle a \text {. }
$$

This equation is equivalent to the condition in Eq. (5.82) above. Note that for selfconsistency, we must have $\left\langle\tilde{n}^{2}\right\rangle=\left\langle\tilde{n}^{2}\right\rangle_{a=0}+\mathcal{O}\left(\chi^{(3)}\right)$. 


\section{General form of the perturbative stochastic scalar model}

Based on above, it is now possible to write down a "general" form of the perturbative model,

$$
\begin{aligned}
\left(h_{0}-\tilde{\chi}^{(1)}+3 \tilde{\chi}^{(3)} \frac{\left\langle n^{2}\right\rangle}{\left(h_{0}-\chi^{(1)}\right)^{2}}\right) x-\tilde{\chi}^{(3)} x^{3} & =a+n, \\
\left(h_{0}-\tilde{\chi}^{(1)}\right)\langle x\rangle-\tilde{\chi}^{(3)}\langle x\rangle^{3} & =a, \\
\langle n\rangle & =0,
\end{aligned}
$$

where the "bare" noise appears. Besides having a zero mean, the higher moments of the noise $n$ can depend in an arbitrary fashion on the external field, as long as it is $\mathcal{O}\left(\chi^{(3)}\right)$. The only other requirement would be adherence to the FDT (see Section (2.1.3)), which pertains to equilibrium $(a=0)$ only.

This justifies the choice $\langle\mathbf{F}\rangle=0$ in Chapters 2 to 4 , as in equilibrium (or in local equilibrium for unequal temperatures) further field dependence of the noise does not enter (in the nonlinear term one uses $\left.\left\langle n^{2}\right\rangle=\left\langle n^{2}\right\rangle_{a=0}+\mathcal{O}\left(\chi^{(3)}\right)\right)$. The question of whether the bare $\left(\chi^{(1)}\right)$ or effective $\left(\tilde{\chi}^{(1)}\right)$ susceptibility remains unchanged can not be addressed from this analysis either, however. We obtain only the difference.

\section{Second and fourth moments of $\delta x$ and Gaussianity}

As seen in the previous chapters, the interesting quantities are not necessarily the stochastic fields or quantities themselves, but rather their moments $\left\langle x^{2}\right\rangle=\langle x\rangle^{2}+\left\langle\delta x^{2}\right\rangle$. From Eq. (5.79), the fluctuations can be written as

$$
\delta x=\frac{n}{\left(h_{0}-\tilde{\chi}^{(1)}\right)}+\chi^{(3)} \frac{3 a^{2} n-3\left\langle n^{2}\right\rangle n+3 a\left[n^{2}-\left\langle n^{2}\right\rangle\right]+\left[n^{3}-\left\langle n^{3}\right\rangle\right]}{\left(h_{0}-\tilde{\chi}^{(1)}\right)^{4}} .
$$

We are interested in the second, third, and fourth moments. To leading order in $\chi^{(3)}$, these are

$$
\begin{aligned}
\left\langle\delta x^{2}\right\rangle & =\frac{\left\langle n^{2}\right\rangle}{\left(h_{0}-\tilde{\chi}^{(1)}\right)^{2}}+6 \chi^{(3)} \frac{a^{2}\left\langle n^{2}\right\rangle}{\left(h_{0}-\tilde{\chi}^{(1)}\right)^{5}} \\
\left\langle\delta x^{3}\right\rangle & =\frac{\left\langle n^{3}\right\rangle}{\left(h_{0}-\tilde{\chi}^{(1)}\right)^{3}}-9 \chi^{(3)} \frac{a\left\langle n^{2}\right\rangle^{2}}{\left(h_{0}-\tilde{\chi}^{(1)}\right)^{6}} \\
\left\langle\delta x^{4}\right\rangle & =\frac{\left\langle n^{4}\right\rangle}{\left(h_{0}-\tilde{\chi}^{(1)}\right)^{4}}+36 \chi^{(3)} \frac{a^{2}\left\langle n^{2}\right\rangle^{2}}{\left(h_{0}-\tilde{\chi}^{(1)}\right)^{7}}+24 \chi^{(3)} \frac{\left\langle n^{2}\right\rangle^{3}}{\left(h_{0}-\tilde{\chi}^{(1)}\right)^{7}}
\end{aligned}
$$




\section{Nonlinear fluctuations in the presence of external fields}

where the Isserlis' theorem $\left(\left\langle n^{4}\right\rangle=\left\langle n^{2}\right\rangle^{2}+\mathcal{O}\left(\chi^{(3)}\right)\right)$ was used in the nonlinear terms. It is interesting to note that these fluctuations cannot be Gaussian. This can be seen trivially from the third moment for non-zero $a$, but it is also true in equilibrium. To see that, we can calculate the "non-Gaussianity" as

$$
\left\langle\delta x^{4}\right\rangle-3\left\langle\delta x^{2}\right\rangle^{2}=\frac{\left\langle n^{4}\right\rangle-3\left\langle n^{2}\right\rangle^{2}}{\left(h_{0}-\tilde{\chi}^{(1)}\right)^{4}}+24 \chi^{(3)} \frac{\left\langle n^{2}\right\rangle^{3}}{\left(h_{0}-\tilde{\chi}^{(1)}\right)^{7}} .
$$

If the fluctuations and noise were Gaussian, then $\left\langle\delta x^{4}\right\rangle-3\left\langle\delta x^{2}\right\rangle^{2}=0$ and $\left\langle n^{4}\right\rangle-$ $3\left\langle n^{2}\right\rangle^{2}=0$. Since the last term does not disappear, however, we must conclude that either $\delta x$ or $n$ (or both) must be non-Gaussian or $\left\langle n^{2}\right\rangle=\mathcal{O}\left(\chi^{(3)}\right)$, which is generally not true because of the FDT.

One should remember that these expressions do not show the complete $a$-dependence explicitly. While the second moment can be determined in equilibrium from FDT, the $\left\langle n^{k}\right\rangle$ can depend arbitrarily on the external force. Possible choices for modelling the noise are given in the next section.

\subsubsection{Modelling non-equilibrium noise}

Without further assumptions, the behavior of noise and fluctuations out of equilibrium still has a lot of freedom. For the third order case, the following condition holds for the average effective noise [Eq. (5.82)],

$$
\langle\tilde{n}\rangle=-3 \chi^{(3)}\left\langle\delta x^{2}\right\rangle_{\langle x\rangle=0}\langle x\rangle .
$$

Alternatively, the bare noise $n$ with a vanishing mean can be used in Eq. (5.91).

In this section we are not particularly interested in the equilibrium fluctuations, which can be fixed through the FDT to some degree (limited to second moment), but rather on how noise or fluctuations depend on the external force $a$ or average field $\langle x\rangle$.

\section{The kinetic form}

Owing to a microscopic description of gas particles, the distribution function is sometimes approximated as being quadratic in momenta $[48,49]$. This is also the route taken in Ref. [43]. This assumption can be leveraged through Focker-Planck equation analysis with the noise taking the following form,

$$
\tilde{n}_{1}=n_{0,1}\left(1+A x^{2}\right),
$$


where $n_{0,1}$ is an independent zero-mean Gaussian noise source. Using Eqs. (5.82) and (5.64), the unknown coefficient $A$ can be solved for. First, setting $a=\langle x\rangle=0$, $n_{0}$ can be expressed through equilibrium $\delta x$ from the effective stochastic equation as

$$
\left[\left(h_{0}-\tilde{\chi}^{(1)}\right) \delta x-\tilde{\chi}^{(3)} \delta x^{3}\right]_{a=0}=n_{0,1} .
$$

Through the condition on the average of the noise, and keeping only leading order terms in $\chi^{(3)}$, we get

$$
\begin{aligned}
\left\langle\tilde{n}_{1}\right\rangle & =A\left\langle n_{0,1} x^{2}\right\rangle \\
& =2 A\langle x\rangle\left\langle\delta x^{2}\right\rangle_{\langle x\rangle=0}\left[h_{0}-\tilde{\chi}^{(1)}-3 \tilde{\chi}^{(3)}\left\langle\delta x^{2}\right\rangle\right] \\
& =-3 \chi^{(3)}\left\langle\delta x^{2}\right\rangle_{\langle x\rangle=0}\langle x\rangle, \\
A & =-\frac{3}{2} \frac{\chi^{(3)}}{\left(h_{0}-\tilde{\chi}^{(1)}\right)} .
\end{aligned}
$$

This noise then becomes

$$
\tilde{n}_{1}=n_{0,1}\left(1-\frac{3}{2} \tilde{\chi}^{(3)} \frac{x^{2}}{h_{0}-\tilde{\chi}^{(1)}}\right),
$$

and the corresponding non-equilibrium fluctuations can be obtained from Eq. (5.77) as

$$
\delta x=\frac{n_{0,1}}{h_{0}-\tilde{\chi}^{(1)}}-\frac{1}{2} \tilde{\chi}^{(3)} \frac{n_{0,1}^{3}}{\left(h_{0}-\tilde{\chi}^{(1)}\right)^{4}}-\frac{1}{2} \tilde{\chi}^{(3)} \frac{a^{2} n_{0,1}}{\left(h_{0}-\tilde{\chi}^{(1)}\right)^{4}} .
$$

Note that since neither $n_{0,1}$ nor $\tilde{\chi}^{(1)}$ depend on $a$, this is the complete dependence, from which one can calculate explicitly the non-equilibrium second, third, and fourth moments as

$$
\begin{aligned}
\left\langle\delta x^{2}\right\rangle & =\frac{\left\langle n_{0,1}^{2}\right\rangle}{\left(h_{0}-\tilde{\chi}^{(1)}\right)^{2}}-\tilde{\chi}^{(3)} \frac{a^{2}\left\langle n_{0,1}^{2}\right\rangle+3\left\langle n_{0,1}^{2}\right\rangle^{2}}{\left(h_{0}-\tilde{\chi}^{(1)}\right)^{5}}, \\
\left\langle\delta x^{3}\right\rangle & =0, \\
\left\langle\delta x^{4}\right\rangle & =\frac{\left\langle n_{0,1}^{4}\right\rangle}{\left(h_{0}+\tilde{\chi}^{(1)}\right)^{4}}-6 \tilde{\chi}^{(3)} \frac{a^{2}\left\langle n_{0,1}^{2}\right\rangle^{2}}{\left(h_{0}+\tilde{\chi}^{(1)}\right)^{7}}-30 \tilde{\chi}^{(3)} \frac{\left\langle n_{0,1}^{2}\right\rangle^{3}}{\left(h_{0}+\tilde{\chi}^{(1)}\right)^{7}} .
\end{aligned}
$$




\section{The minimal form}

As a contrasting case, let's take the $n$ in Eq. (5.91) as a completely independent source of noise. As shown above, we have the equation

$$
\left(h_{0}-\tilde{\chi}^{(1)}+3 \tilde{\chi}^{(3)} \frac{\left\langle n_{0,2}^{2}\right\rangle}{\left(h_{0}+\tilde{\chi}^{(1)}\right)^{2}}\right) x-\tilde{\chi}^{(3)} x^{3}=a+n_{0,2} .
$$

This corresponds to an effective noise

$$
\tilde{n}_{2}=n_{0,2}-3 \tilde{\chi}^{(3)}\left\langle\delta x^{2}\right\rangle x
$$

Compared to the "kinetic" form of the effective noise $\tilde{n}_{1}$ in Eq. $5.104, \tilde{n}_{2}$ has a linear term in $x$, as opposed to a quadratic one. The corresponding non-equilibrium fluctuations can be obtained from Eq. (5.77) as

$$
\delta x=\frac{n_{0,2}}{h_{0}-\tilde{\chi}^{(1)}}+\frac{\tilde{\chi}^{(3)}}{\left(h_{0}-\tilde{\chi}^{(1)}\right)^{4}}\left(3 a^{2} n_{0,2}+3 a n_{0,2}^{2}-3 a\left\langle n_{0,2}^{2}\right\rangle-3\left\langle n_{0,2}^{2}\right\rangle n_{0,2}+n_{0,2}^{3}\right) .
$$

The second, third, and fourth moments are then

$$
\begin{aligned}
\left\langle\delta x^{2}\right\rangle & =\frac{\left\langle n_{0,2}^{2}\right\rangle}{\left(h_{0}-\tilde{\chi}^{(1)}\right)^{2}}+6 \chi^{(3)} \frac{a^{2}\left\langle n_{0,2}^{2}\right\rangle}{\left(h_{0}-\tilde{\chi}^{(1)}\right)^{5}}, \\
\left\langle\delta x^{3}\right\rangle & =-9 \chi^{(3)} \frac{a\left\langle n_{0,2}^{2}\right\rangle^{2}}{\left(h_{0}-\tilde{\chi}^{(1)}\right)^{6}}, \\
\left\langle\delta x^{4}\right\rangle & =\frac{\left\langle n_{0,2}^{4}\right\rangle}{\left(h_{0}-\tilde{\chi}^{(1)}\right)^{4}}+36 \chi^{(3)} \frac{a^{2}\left\langle n_{0,2}^{2}\right\rangle^{2}}{\left(h_{0}-\tilde{\chi}^{(1)}\right)^{7}}+24 \chi^{(3)} \frac{\left\langle n_{0,2}^{2}\right\rangle^{3}}{\left(h_{0}-\tilde{\chi}^{(1)}\right)^{7}} .
\end{aligned}
$$

\section{Comparison and remarks}

Comparing the fluctuations obtained from these two models, obvious differences can be seen. Due to FDT, the equilibrium second moment $\left\langle\delta x^{2}\right\rangle_{\langle x\rangle=0}$ must be equal for both (the equilibrium linear response is given by $\tilde{\chi}^{(1)}$, which is the same). This gives a relation between the second moments of $n_{0,1}$ and $n_{0,2}$,

$$
\begin{aligned}
& \left\langle n_{0,1}^{2}\right\rangle=\left\langle n_{0,2}^{2}\right\rangle+\chi^{(3)} \frac{3\left\langle n_{0,2}^{2}\right\rangle^{2}}{\left(h_{0}-\tilde{\chi}^{(1)}\right)^{3}}, \\
& \left\langle n_{0,2}^{2}\right\rangle=\left\langle n_{0,1}^{2}\right\rangle-\chi^{(3)} \frac{3\left\langle n_{0,1}^{2}\right\rangle^{2}}{\left(h_{0}-\tilde{\chi}^{(1)}\right)^{3}} .
\end{aligned}
$$


Note that this difference is of order $\chi^{(3)}$. The non-equilibrium second moments $\left\langle\delta x^{2}\right\rangle$ can now be compared directly,

$$
\left\langle\delta x_{1}^{2}\right\rangle-\left\langle\delta x_{2}^{2}\right\rangle=7 \chi^{(3)} \frac{a^{2}\left\langle n_{0,1}^{2}\right\rangle}{\left(h_{0}-\tilde{\chi}^{(1)}\right)^{5}} .
$$

The second moments of the fluctuations are equal in equilibrium by design (it is given by the linear response, which must be same for identical systems), but the two models predict a different behavior out of equilibrium. Comparing higher moments is more difficult, because $n_{0,1}$ and $n_{0,2}$ are not necessarily Gaussian, which means the relation between $\left\langle n_{0,1}^{4}\right\rangle$ and $\left\langle n_{0,2}^{4}\right\rangle$ is unknown. (A possible route is to enforce the equality of all moments in equilibrium.)

On one hand, the different results obtained from these models reflects a lack of predictive power. On the other hand, this framework lends itself nicely to making informed assumptions about the underlying physics. The exact form of the noise depends, at least on this level, on unknown microscopic details. The conditions obtained here and in the previous section must nevertheless be fulfilled, even if the underlying physics is clarified or better theoretical tools developed.

\subsection{Nonlinear fluctuational electrodynamics with external fields}

As seen in Sections 5.2 and 5.3, the non-equilibrium behavior of the fluctuations cannot be determined without further assumptions on how the noise depends on the external field. The goal of this section is to show how the fluctuations depend on the external field for a particular choice of this dependence, that still obeys the restrictions outlined above (particularly Eq. (5.14)).

Since in equilibrium we studied the case of $\langle\mathbf{F}\rangle=0$ (see Section 2.4), a natural extension out of equilibrium is similar to the "minimal form" in Eq. (5.107). The analogue for (5.106) is the bare equation 2.116,

$$
\left(\mathbb{H}_{0}-\mathbb{V}\right) \mathbf{E}-\mathcal{M}[\mathbf{E} \otimes \mathbf{E}]-\mathcal{N}[\mathbf{E} \otimes \mathbf{E} \otimes \mathbf{E}]=\mathbb{H}_{0} \mathbf{E}_{\text {in }}+\mathbf{F},
$$

where the noise in equilibrium is given by Eq. 3.6 as

$$
\left\langle\mathbf{F}_{\omega} \otimes \mathbf{F}_{\omega^{\prime}}^{*}\right\rangle^{\mathrm{eq}}=-\delta\left(\omega-\omega^{\prime}\right) \frac{b(\omega)}{2 i}\left(\mathbb{H}_{0}-\tilde{\mathbb{V}}^{\mathrm{eq}}\right)_{\mathrm{AH}} .
$$

In order to make the minimal extension into non-equilibrium, we assume that the noise correlator does not depend on the field, that the noise $\mathbf{F}$ is the analogue of $n_{0,2}$ in 
5. Nonlinear fluctuations in the presence of external fields

(5.106).

By subtracting its own average from Eq. (5.115), we obtain an expression for the fluctuations $\delta \mathbf{E}=\mathbf{E}-\overline{\mathbf{E}}$ as

$$
\begin{aligned}
\left(\mathbb{H}_{0}-\mathbb{V}\right) \delta \mathbf{E} & =\mathbf{F} \\
& +\mathcal{M}[\delta \mathbf{E} \otimes \delta \mathbf{E}-\langle\delta \mathbf{E} \otimes \delta \mathbf{E}\rangle] \\
& +\mathcal{N}[\delta \mathbf{E} \otimes \delta \mathbf{E} \otimes \delta \mathbf{E}-\langle\delta \mathbf{E} \otimes \delta \mathbf{E} \otimes \delta \mathbf{E}\rangle] \\
& +\mathcal{N}[3 \overline{\mathbf{E}} \otimes \overline{\mathbf{E}} \otimes \delta \mathbf{E}] \\
& +\mathcal{M}[2 \overline{\mathbf{E}} \otimes \delta \mathbf{E}]+\mathcal{N}[3 \overline{\mathbf{E}} \otimes \delta \mathbf{E} \otimes \delta \mathbf{E}-3 \overline{\mathbf{E}} \otimes\langle\delta \mathbf{E} \otimes \delta \mathbf{E}\rangle]
\end{aligned}
$$

Keeping only first order terms in $\chi^{(2)}$ and $\chi^{(3)}$, as before, and using

$$
\mathbf{F}=\left(\mathbb{H}_{0}-\mathbb{V}\right) \delta \mathbf{E}+\mathcal{O}\left(\chi^{(2)}\right)+\mathcal{O}\left(\chi^{(3)}\right)
$$

we obtain

$$
\begin{aligned}
& \left(\mathbb{H}_{0}-\mathbb{V}\right)\left\langle\delta \mathbf{E} \otimes \delta \mathbf{E}^{*}\right\rangle\left(\mathbb{H}_{0}-\mathbb{V}\right)^{\dagger}=\left\langle\mathbf{F} \otimes \mathbf{F}^{*}\right\rangle \\
& +3 \mathcal{N}[\langle\delta \mathbf{E} \otimes \delta \mathbf{E}\rangle]\left\langle\delta \mathbf{E} \otimes \delta \mathbf{E}^{*}\right\rangle\left(\mathbb{H}_{0}-\mathbb{V}\right)^{\dagger} \\
& +3\left\langle\mathcal{N}[\overline{\mathbf{E}} \otimes \overline{\mathbf{E}}]\left\langle\delta \mathbf{E} \otimes \delta \mathbf{E}^{*}\right\rangle\left(\mathbb{H}_{0}-\mathbb{V}\right)^{\dagger}\right\rangle \\
& +2\left\langle\mathcal{M}[\overline{\mathbf{E}}]\left\langle\delta \mathbf{E} \otimes \delta \mathbf{E}^{*}\right\rangle\left(\mathbb{H}_{0}-\mathbb{V}\right)^{\dagger}\right\rangle \\
& +3\left(\mathbb{H}_{0}-\mathbb{V}\right)\left\langle\delta \mathbf{E} \otimes \delta \mathbf{E}^{*}\right\rangle \mathcal{N}[\langle\delta \mathbf{E} \otimes \delta \mathbf{E}\rangle]^{\dagger} \\
& +3\left(\mathbb{H}_{0}-\mathbb{V}\right)\left\langle\delta \mathbf{E} \otimes \delta \mathbf{E}^{*}\right\rangle \mathcal{N}[\overline{\mathbf{E}} \otimes \overline{\mathbf{E}}]^{\dagger} \\
& +2\left(\mathbb{H}_{0}-\mathbb{V}\right)\left\langle\delta \mathbf{E} \otimes \delta \mathbf{E}^{*}\right\rangle \mathcal{M}[\overline{\mathbf{E}}]^{\dagger} \text {. }
\end{aligned}
$$

It can be seen that $\langle\delta \mathbf{E} \otimes \delta \mathbf{E}\rangle=\langle\delta \mathbf{E} \otimes \delta \mathbf{E}\rangle^{\mathrm{eq}}+\mathcal{O}\left(\chi^{(2)}\right)+\mathcal{O}\left(\chi^{(3)}\right)$. The above relation can then be rearranged as

$$
\left(\mathbb{H}_{0}-\tilde{\mathbb{V}}_{\overline{\mathbf{E}}}\right)\left\langle\delta \mathbf{E} \otimes \delta \mathbf{E}^{*}\right\rangle\left(\mathbb{H}_{0}-\tilde{\mathbb{V}}_{\overline{\mathbf{E}}}\right)^{\dagger}=\left\langle\mathbf{F} \otimes \mathbf{F}^{*}\right\rangle,
$$

where the effective non-equilibrium potential appears,

$$
\tilde{\mathbb{V}}_{\overline{\mathbf{E}}}=\mathbb{V}+3 \mathcal{N}\left[\langle\delta \mathbf{E} \otimes \delta \mathbf{E}\rangle^{\mathrm{eq}}\right]+2 \mathcal{M}[\overline{\mathbf{E}}]+3 \mathcal{N}[\overline{\mathbf{E}} \otimes \overline{\mathbf{E}}] .
$$

This quantity can be viewed as a potential, because the effective Helmholtz equation [Eq. (2.123)] can be written as

$$
\left(\mathbb{H}_{0}-\tilde{\mathbb{V}}_{\overline{\mathbf{E}}}\right) \overline{\mathbf{E}}=\mathbb{H}_{0} \mathbf{E}_{\text {in }}
$$


It represents the linear response at a finite average field. In equilibrium the known effective potential [see Eq. (3.12)] is recovered, $\tilde{\mathbb{V}}_{\overline{\mathbf{E}} \rightarrow 0}=\tilde{\mathbb{V}}^{\mathrm{eq}}$.

Letting $\left\langle\mathbf{F} \otimes \mathbf{F}^{*}\right\rangle=\left\langle\mathbf{F} \otimes \mathbf{F}^{*}\right\rangle^{\text {eq }}$ from Eq. (5.116) as intended, the non-equilibrium fluctuations can be obtained explicitly:

$$
\begin{aligned}
\left\langle\delta \mathbf{E} \otimes \delta \mathbf{E}^{*}\right\rangle & =-\delta\left(\omega-\omega^{\prime}\right) \frac{b(\omega)}{2 i}\left(\mathbb{H}_{0}-\tilde{\mathbb{V}}_{\overline{\mathbf{E}}}\right)^{-1}\left(\mathbb{H}_{0}-\tilde{\mathbb{V}}^{\mathrm{eq}}\right)_{\mathrm{AH}}\left(\mathbb{H}_{0}-\tilde{\mathbb{V}}_{\overline{\mathbf{E}}}\right)^{-1 \dagger} \\
& =\delta\left(\omega-\omega^{\prime}\right) \frac{b(\omega)}{2 i}\left[\tilde{\mathbb{G}}_{\mathrm{AH}}\right] \\
& +\delta\left(\omega-\omega^{\prime}\right) \frac{b(\omega)}{2 i}[\tilde{\mathbb{G}}(2 \mathcal{M}[\overline{\mathbf{E}}]+3 \mathcal{N}[\overline{\mathbf{E}} \otimes \overline{\mathbf{E}}]) \tilde{\mathbb{G}}]_{\mathrm{AH}} \\
& -\delta\left(\omega-\omega^{\prime}\right) \frac{b(\omega)}{2 i}\left[\tilde{\mathbb{G}}(2 \mathcal{M}[\overline{\mathbf{E}}]+3 \mathcal{N}[\overline{\mathbf{E}} \otimes \overline{\mathbf{E}}])_{\mathrm{AH}} \tilde{\mathbb{G}}^{\dagger}\right] \\
& =\delta\left(\omega-\omega^{\prime}\right) \frac{b(\omega)}{2 i}\left[\tilde{A}_{\overline{\mathbf{E}}} \tilde{\mathbb{G}}_{\mathrm{AH}} \mathbb{A}_{\overline{\mathbf{E}}}^{\dagger}\right] \\
\mathbb{A}_{\overline{\mathbf{E}}} & =\mathbb{I}+2 \tilde{\mathbb{G}} \mathcal{M}[\overline{\mathbf{E}}]+3 \tilde{\mathbb{G}} \mathcal{N}[\overline{\mathbf{E}} \otimes \overline{\mathbf{E}}]
\end{aligned}
$$

where $\tilde{\mathbb{G}}=\left(\mathbb{H}_{0}-\tilde{\mathbb{V}}^{e q}\right)^{-1}$. As can be seen, the nonlinear fluctuations grow with the external field. However, since the full field correlator is $\left\langle\mathbf{E} \otimes \mathbf{E}^{*}\right\rangle=\left\langle\delta \mathbf{E} \otimes \delta \mathbf{E}^{*}\right\rangle+\overline{\mathbf{E}} \otimes \overline{\mathbf{E}}^{*}$, the deterministic part cannot be overcome through an increase in the scale of $\overline{\mathbf{E}}$. Instead experimental situations should be considered, where the deterministic effects vanish. An example of this is the dipole-field configuration in Figure 5.1. Another example could be field-modified heat radiation spectra, in the spirit of Ref. [43], for general systems.

\subsection{Summary}

This chapter was light on physical predictions and focused more on carefully peeling off as many degrees of freedom from the noise. What we end up with, is quite a robust framework for studying different kinds of physical constraints (for example due to microscopic detail) or assumptions on the noise, while keeping the theory self-consistent and at the same time grounded in measurable quantities (the response of the mean to an external force).

To test the formalism, we applied two different assumptions on how the noise could depend on the stochastic variable (linear or quadratic), one motivated from the work on electrodynamics and the other from nonlinear Brownian motion. Both are valid choices from the viewpoint of this chapter, but they give different fluctuations out of equilibrium. On the bright side, this gives a tool for comparing both of these assumptions directly with the experiment. 
5. Nonlinear fluctuations in the presence of external fields

As a proof of concept, we demonstrated how the findings can be generalized for the electromagnetic field. The simplest noise, which can be determined completely from equilibrium, leads to very clear field dependence of the fluctuations. This result can be used to calculate the Casimir force or, with the addition of the LTE approximation, radiative heat transfer. Therefore this assumption can also be tested directly from experiment. 


\section{Concluding remarks}

\subsection{Results and conclusions}

This project started four years ago with a deceptively simple goal: to develop fluctuational electrodynamics for nonlinear materials, with "tunable" Casimir forces and heat radiation in mind. We only had two tools, the nonlinear Helmholtz equation (albeit with an unknown noise term) and the FDT. By using the FDT as a foundation, the theory was always focused strictly on measurable quantities: the linear response, corresponding to a scattering experiment, and the field correlator, corresponding to the Casimir force and heat radiation. In this sense, almost all of the results given in this thesis correspond to directly observable quantities from experiments.

In order to make predictions, assumptions generally have to be made within any model. As we saw in Chapter 5, the FDT on its own leaves quite a lot of freedom in the model if no other restrictions apply. It is visible through Chapters 3,4 , and 5 , that as we go from equilibrium, out of thermal equilibrium, to full non-equilibrium case, the more egregious the necessary assumptions become, since the theory moves farther and farther away from the FDT. Nevertheless, reasonable choices were suggested and corresponding predictions obtained. In the end, the ultimate test of the validity of our assumptions (and thus the value of this part of the theory) can only come from experiments.

The formula for the equilibrium Casimir force between parallel nonlinear plates (the most well-known and easily measurable effect) obtained in the Master's thesis [83] forms the basis of a refined equilibrium fluctuational electrodynamics for nonlinear materials, which was consolidated in Ref. [85] and outlined here in Chapter 3. It was shown that the interesting properties (the linear response and field correlator) depend on an effective dielectric function, which, in turn, depends on fluctuations. Since all parts of a system are sources for fluctuations, the effective dielectric function depends on boundaries (becoming inhomogeneous near them) as well as the locations and properties of other objects. It is this dependence on relative locations that causes the crossovers from well-known power laws of the Casimir force between nonlinear parallel plates.

The step to break equilibrium in Chapter 4 within the local equilibrium approximation was a small one, following the same line of thought familiar from linear FE while taking 


\section{Concluding remarks}

care of the effective properties. As expected, since the non-equilibrium fluctuations change (the FDT no longer holds), so too does the effective dielectric function. In fact, it depends on the temperature difference between all other objects. Surprisingly, though, this contribution turned out to change its sign under the change of temperatures if a single object was considered. This makes it possible for a passive medium to become pseudo-active, at least for some frequency range, amplifying fields at those frequencies propagating through the material. This curiosity was demonstrated to have an even more surprising effect on the heat radiation of a nanosphere that is non-absorbing in equilibrium: it could never absorb more heat than it radiates away, thus cooling even if it is at a lower temperature than the environment.

Incorporating external fields, with the end goal of tunable Casimir forces and heat radiation, was tackled in Chapter 5. This turned out to be quite challenging due to a lack of information regarding non-equilibrium behavior in the FDT itself. Using a scalar model for simplicity, it was shown how the noise in an effective model cannot be completely freely chosen. In a perturbative treatment this condition gives a non-zero mean of the effective noise, which can be incorporated into the bare coefficients (as was done in previous chapters implicitly). While this does not fix completely the second moment of the noise, which is necessary to predict the non-equilibrium Casimir effect or heat radiation, it does create a useful framework for additional physically motivated conditions on the noise. Two of these were compared for the scalar model: the kinetic model (effective noise is quadratic in field) and the minimal model (effective noise is linear in field, but bare noise remains independent), which lead to different field dependence out of equilibrium. For the latter case, we also gave an expression for the field-dependent electromagnetic fluctuations.

\subsection{Open questions}

The nature of this work is inherently exploratory - nonlinear fluctuational electrodynamics is niche topic (no prior research) and there are no preexisting experimental results to guide theoretical frameworks. Therefore, together with predictions for experiments, this thesis also raises a lot of new questions which remain beyond the scope of this work.

One of these questions is the distinction between the bare and effective quantities and which one should remain constant. This could be tested through linear response measurements under changing conditions: either a two-object system with variable distance or a single object at constant temperature with variable environmental temperature. A change in the measured dielectric function in either case would indicate that the bare properties remain constant. This question could also be answered by considering 
microscopic theories.

Another problem concerns higher order moments of the noise and field. The standard FDT gives directly only the second moment, and while this is enough for perturbative treatment (Gaussianity can be assumed in nonlinear terms where the higher moments appear), extending the theory to higher orders in terms of the nonlinear susceptibilities would further necessitate a way of calculating higher order moments.

Furthermore, while some restriction on the dependence of noise on the external field was established, notably the first moment in perturbative case, the behavior of higher order moments remains unknown in general. Whether these conditions can be narrowed through general theoretical considerations or microscopic detail is unclear. However, the formalism allows for straightforward comparison of different assumptions with experiment or microscopic calculations.

\subsection{Outlook}

Besides clarifying the remaining questions above, there are very clear paths for future research that would benefit from and extend the current results. This is especially relevant in the context of increased interest in the nature of non-equilibrium fluctuations in nonlinear systems. Furthermore, due to the weakness of optical nonlinearities in currently available materials, it would be of great interest to isolate effects that are absent in purely linear systems.

Most prominently, while the formalism was applied for the Casimir force and heat radiation in simple geometries as examples, different setups and effects present an attractive avenue for future work. For viable short range measurements, for example, it is necessary to give results for the sphere-plate geometry (representing the tip of an atomic force microscope and the substrate). Other configurations could be of interest to eliminate or minimize linear or deterministic effects, in the spirit of the "magic angle" of Figure 5.1.

In addition to different geometries, effects beside Casimir force and heat radiation could be of interest. This would include quantum friction that particles moving near surfaces experience [66] as well as extending the studies to tunable (field-dependent) heat radiation and Casimir force. Furthermore, the emergence of effective gain media and possible effective non-reciprocity induced by thermal imbalance (see Chapter 4 ) could have very interesting experimental applications $[6,94,82]$.

The formalism can also be extended to include non-local effects by allowing the susceptibilities depend on not only frequencies but also the k-vector. Most notably, this 


\section{Concluding remarks}

would allow for the treatment of plasmas, which are strongly nonlinear, but inherently non-local $[93,31,30]$. This is also possibly a viable system to model microscopically.

While the list of open questions and research ideas looks daunting, the author hopes that future years will shed more light on the many as of yet unexplored aspects of electromagnetic fluctuations in nonlinear systems. Especially with the ever advancing experimental capabilities, it is a field of great potential to both theory as well as application. 


\section{A. Functional derivatives}

A general functional derivative is defined as

$$
\begin{aligned}
& \frac{\delta \mathcal{A}(\mathbf{B})}{\delta \mathbf{B}}=\delta_{\mathbf{B}} \mathcal{A}=\mathcal{A}^{\prime}, \\
& \mathcal{A}^{\prime}(\mathbf{F})=\lim _{\epsilon \rightarrow 0} \frac{\mathcal{A}(\mathbf{B}+\varepsilon \mathbf{F})-\mathcal{A}(\mathbf{B})}{\epsilon} \forall \mathbf{F},
\end{aligned}
$$

where $\mathcal{A}$ or $\mathbf{B}$ could be any vector or tensor or field. For example if $\mathcal{A}(\mathbf{B}), \mathbf{B} \in \mathbb{R}$, then we recover the regular definition of a derivative. On the other hand, an action would be $\mathcal{F}[\phi(\mathbf{r})] \in \mathbb{R}$, where the arguments are functions themselves, $\phi \in\{\mathbb{R} \rightarrow \mathbb{R}\}$. In that case, we recover the well known formula [3],

$$
\delta_{\phi} \mathcal{F}[f(\mathbf{r})]=\int_{\mathbb{R}} \mathrm{d} \mathbf{r} \delta_{\phi} \mathcal{F}(\mathbf{r}) f(\mathbf{r})=\lim _{\epsilon \rightarrow 0} \frac{\mathcal{F}[\phi(\mathbf{r})+\epsilon f(\mathbf{r})]-\mathcal{F}[\phi(\mathbf{r})]}{\epsilon} .
$$

This is, however, not enough for the purposes of this thesis. $\mathcal{A}$ needs to be a function mapping tensor fields and operators to tensor fields or operators, because we want to study how the electric field (or composed tensors such as $\mathbf{E} \otimes \mathbf{E}$ ) change under small changes of the external field. A generic notation is useful, because quite often it is not necessary to use Eq. (A.2) directly. Instead, analogues to regular derivative rules (such as the chain rule) often apply in more complicated cases with no or minor differences $[3]$.

\section{Representing a functional as a series}

We define a vector field $\mathbf{A}$, with components $A_{i} \rightarrow A_{i}(\mathbf{r}, \omega)$ which include the directional indices $\{1,2,3\}$, the spatial coordinate $\mathbf{r}$ and frequency $\omega$. We consider it to be a function of a similar vector field $\mathbf{B}$,

$$
\begin{aligned}
A_{i}(\mathbf{B}) & =A_{i}^{(0)}+A_{i j}^{(1)} B_{j}+A_{i j k}^{(2)} B_{j} B_{k}+A_{i j k l}^{(3)} B_{j} B_{k} B_{l}+\ldots \\
& =\sum_{n=0}^{\infty} A_{i j_{1} . . j_{n}}^{(n)} \prod_{m=1}^{n} B_{j_{m}} .
\end{aligned}
$$




\section{A. Functional derivatives}

Notice that since the components $B_{j}$ commute, the coefficients $A_{i j_{1} . . j_{n}}^{(n)}$ can be considered symmetric under the exchange of any two $j_{k}$ indices. The first functional derivative is then

$$
\left(\frac{\delta \mathbf{A}}{\delta \mathbf{B}}\right)_{i j} \mathbf{f}_{j}=\lim _{\epsilon \rightarrow 0} \frac{\sum_{n=0}^{\infty} A_{i j_{1 . . j}}^{(n)} \prod_{m=1}^{n}\left(B_{j_{m}}+\epsilon f_{j_{m}}\right)-\sum_{n=0}^{\infty} A_{i j_{1 . . j}}^{(n)} \prod_{m=1}^{n} B_{j_{m}}}{\epsilon} .
$$

In the denominator only terms linear in $\epsilon$ survive. Taking also into account the symmetry in $A_{i_{0} . . i_{n}}^{(n)}$, we arrive at

$$
\begin{aligned}
\left(\frac{\delta \mathbf{A}}{\delta \mathbf{B}}\right)_{i j} \mathbf{f}_{j} & =A_{i j_{1}}^{(1)} n f_{j_{1}}+\sum_{n=2}^{\infty} A_{i j_{1} . . j_{n}}^{(n)} n f_{j_{1}} \prod_{m=2}^{n} B_{j_{m}} \\
& =\left(A_{i j_{1}}^{(1)}+\sum_{n=2}^{\infty} n A_{i j_{1} . . j_{n}}^{(n)} \prod_{m=2}^{n} B_{j_{m}}\right) f_{i_{1}} .
\end{aligned}
$$

Therefore, explicitly, the first derivative is

$$
\left(\frac{\delta \mathbf{A}}{\delta \mathbf{B}}\right)_{i j}=A_{i j}^{(1)}+\sum_{n=2}^{\infty} n A_{i j j_{2} . . j_{n}}^{(n)} \prod_{m=2}^{n} B_{j_{m}} .
$$

This can be easily continued to obtain

$$
\left(\frac{\delta^{k} \mathbf{A}}{\delta \mathbf{B}^{k}}\right)_{i j_{1} . . j_{k}}=k ! A_{i j_{1} . . j_{k}}^{(k)}+\sum_{n=k+1}^{\infty} \frac{n !}{(n-k) !} A_{i j_{1} j_{2} . . j_{n}}^{(n)} \prod_{m=k}^{n} B_{j_{m}} .
$$

Just as in the regular Taylor series analysis, the coefficient tensors of $\mathbf{A}$ are directly related to the same order functional derivatives in the limit $\mathbf{B} \rightarrow 0$. 


\section{B. Green's functions for multilayer structures}

\section{B.1. Green's function for two parallel surfaces}

Using the geometry shown in Figure 3.5, with both points inside plate 1 and the other plate (3) a distance $d$ away in the z-direction, the Green's function is given as [39]

$$
\begin{aligned}
\mathbb{G}\left(\mathbf{r}_{l=1}>\mathbf{r}_{l^{\prime}=1}^{\prime}\right) & =\frac{i}{2} \sum_{\sigma} \int \frac{\mathrm{d}^{2} \mathbf{q}_{\|}}{(2 \pi)^{2}} \frac{1}{p_{1}} e^{i \mathbf{q}_{1}^{+} \cdot \mathbf{r}} \hat{\sigma}_{1}^{+} \\
& \otimes\left[e^{-i \mathbf{q}_{1}^{+} \cdot \mathbf{r}^{\prime}} \hat{\sigma}_{1}^{+}+\frac{\left[\mathcal{F}_{12}^{\sigma} e^{-i p_{2} d}+\mathcal{F}_{23}^{\sigma} e^{i p_{2} d}\right]}{\left[e^{-i p_{2} d}+\mathcal{F}_{12}^{\sigma} \mathcal{F}_{23}^{\sigma} e^{i p_{2} d}\right]} e^{-i \mathbf{q}_{1}^{-} \cdot \mathbf{r}^{\prime}} \hat{\sigma}_{1}^{-}\right] \\
& -\frac{\hat{\mathbf{z}} \otimes \hat{\mathbf{z}}}{k_{l}^{2}} \delta^{(3)}\left(\mathbf{r}-\mathbf{r}^{\prime}\right) .
\end{aligned}
$$

This is for the case $z>z^{\prime}$, which does not matter, because we are interested in the limit $\mathbf{r} \rightarrow \mathbf{r}^{\prime}$. The various quantities are

$$
\begin{aligned}
p_{l} & =\sqrt{k_{l}^{2}-q_{\|}^{2}}, \\
k_{l}^{2} & =\frac{\omega^{2}}{v_{l}^{2}}=\varepsilon_{l} \frac{\omega^{2}}{c^{2}}, \\
\mathbf{q}_{l}^{\tau} & =\mathbf{q}_{\|}+\tau p_{l} \hat{\mathbf{z}}=\left(q_{\|} \cos \phi_{q}, q_{\|} \sin \phi_{q}, \tau p_{l}\right), \\
\hat{\mathbf{q}}_{l}^{\tau} & =\frac{\mathbf{q}_{l}^{\tau}}{k_{l}}, \\
\hat{\mathbf{s}}_{l}^{\tau} & =\frac{\hat{\mathbf{z}} \times \hat{\mathbf{q}}_{l}^{\tau}}{\left|\hat{\mathbf{z}} \times \hat{\mathbf{q}}_{l}^{\tau}\right|}=\left(-\sin \phi_{q}, \cos \phi_{q}, 0\right), \\
\hat{\mathbf{p}}_{l}^{\tau} & =\hat{\mathbf{s}}_{l}^{\tau} \times \hat{\mathbf{q}}_{l}^{\tau}=\frac{1}{k_{l}}\left(\tau p_{l} \cos \phi_{q}, \tau p_{l} \sin \phi_{q},-q_{\|}\right), \\
\hat{\mathbf{q}}_{l}^{\tau} \times \hat{\mathbf{s}}_{l}^{\tau} & =-\hat{\mathbf{p}}_{l}^{\tau}, \\
\hat{\mathbf{q}}_{l}^{\tau} \times \hat{\mathbf{p}}_{l}^{\tau} & =\hat{\mathbf{s}}_{l}^{\tau} .
\end{aligned}
$$




\section{B. Green's functions for multilayer structures}

This is a very useful basis since it is diagonal when integrated in the xy-plane.

$$
\begin{aligned}
\int_{0}^{2 \pi} \mathrm{d} \phi_{q} \hat{\mathbf{s}}^{ \pm} \otimes \hat{\mathbf{s}}^{ \pm} & =2 \pi\left(\begin{array}{ccc}
\frac{1}{2} & 0 & 0 \\
0 & \frac{1}{2} & 0 \\
0 & 0 & 0
\end{array}\right) \\
\int_{0}^{2 \pi} \mathrm{d} \phi_{q} \hat{\mathbf{p}}_{l}^{+} \otimes \hat{\mathbf{p}}_{l}^{ \pm} & =2 \pi \frac{p_{l}^{2}}{k_{l}^{2}}\left(\begin{array}{ccc} 
\pm \frac{1}{2} & 0 & 0 \\
0 & \pm \frac{1}{2} & 0 \\
0 & 0 & \frac{q_{\|}^{2}}{p_{l}^{2}}
\end{array}\right) .
\end{aligned}
$$

The Fresnel coefficients are given as

$$
\begin{aligned}
& \mathcal{F}_{l n}^{s} \rightarrow \frac{p_{1}-p_{2}}{p_{1}+p_{2}}=\frac{1-\sqrt{\frac{\varepsilon_{2}}{\varepsilon_{1}}}}{1+\sqrt{\frac{\varepsilon_{2}}{\varepsilon_{1}}}}, \\
& \mathcal{F}_{l n}^{p} \rightarrow \frac{\varepsilon_{2} p_{1}-\varepsilon_{1} p_{2}}{\varepsilon_{2} p_{1}+\varepsilon_{1} p_{2}}=\frac{\varepsilon_{2}-\varepsilon_{1} \sqrt{\frac{\varepsilon_{2}}{\varepsilon_{1}}}}{\varepsilon_{2}+\varepsilon_{1} \sqrt{\frac{\varepsilon_{2}}{\varepsilon_{1}}}}
\end{aligned}
$$

And the bulk or single-surface Green's functions are recovered by setting either both or one of the Fresnel coefficients $\left(\mathcal{F}_{12}^{\sigma}, \mathcal{F}_{23}^{\sigma}\right)$, zero.

\section{B.2. $\operatorname{Im} \mathbb{G}(\mathbf{r}, \mathbf{r})$ in bulk}

The $\delta^{(3)}\left(\mathbf{r}-\mathbf{r}^{\prime}\right)$ contribution disappears, because it is real. For the imaginary part, we have

$$
\begin{aligned}
\operatorname{Im} \mathbb{G}_{\text {bulk }}\left(\mathbf{r}_{l=1}=\mathbf{r}_{l^{\prime}=1}^{\prime}\right) & =\operatorname{Re} \frac{1}{2} \sum_{\sigma} \int \frac{\mathrm{d}^{2} \mathbf{q}_{\|}}{(2 \pi)^{2}} \frac{1}{p_{1}} \hat{\sigma}_{1}^{+} \otimes \hat{\sigma}_{1}^{+} \\
& =\operatorname{Re} \frac{1}{8 \pi^{2}} \sum_{\sigma} \int_{0}^{\infty} \mathrm{d} q_{\|} \frac{q_{\|}}{p_{1}} \int_{0}^{2 \pi} \mathrm{d} \phi_{q} \hat{\sigma}_{1}^{+} \otimes \hat{\sigma}_{1}^{+} \\
& =\operatorname{Re} \frac{1}{8 \pi} \frac{1}{k_{1}^{2}} \int_{0}^{\infty} \mathrm{d} q_{\|} \frac{q_{\|}}{\sqrt{k_{1}^{2}-q_{\|}^{2}}}\left(\begin{array}{ccc}
2 k_{1}^{2}-q_{\|}^{2} & 0 & 0 \\
0 & 2 k_{1}^{2}-q_{\|}^{2} & 0 \\
0 & 0 & 2 q_{\|}^{2}
\end{array}\right) .
\end{aligned}
$$

The integrals $\int_{0}^{\infty} \mathrm{d} q_{\|} \frac{q_{\|}}{\sqrt{k_{1}^{2}-q_{\|}^{2}}}$ and $\int_{0}^{\infty} \mathrm{d} q_{\|} \frac{q_{\|}^{3}}{\sqrt{k_{1}^{2}-q_{\|}^{2}}}$ are in general infinite and the above expression diverges. 
If $\varepsilon_{1}$ is real, however, the integrand is real only for $q_{\|}<k_{1}$. Then it is enough to integrate in the finite range, and we get the finite results $\int_{0}^{k_{1}} \mathrm{~d} q_{\|} \frac{q_{\|}}{\sqrt{k_{1}^{2}-q_{\|}^{2}}}=k_{1}, \int_{0}^{\infty} \mathrm{d} q_{\|} \frac{q_{\|}^{3}}{\sqrt{k_{1}^{2}-q_{\|}^{2}}}=$ $\frac{2}{3} k_{1}^{3}$. We thus obtain

$$
\operatorname{Im} \mathbb{G}\left(\mathbf{r}_{l=1}=\mathbf{r}_{l^{\prime}=1}^{\prime}\right)=\frac{1}{6 \pi} k_{1} .
$$

In vacuum, with $\varepsilon_{1}=1$, we recover the well-known environment dust,

$$
\lim _{\mathbf{r} \rightarrow \mathbf{r}^{\prime}} \operatorname{Im} \mathbb{G}_{0}\left(\mathbf{r}, \mathbf{r}^{\prime}\right)_{i j}=\frac{1}{6 \pi} \frac{\omega}{c} \delta_{i j}
$$

\section{B.3. $\operatorname{Im} \mathbb{G}(\mathbf{r}, \mathbf{r})$ for a single surface}

Here we give the difference between a single surface and homogeneous case (start from two touching plates, then move one very far away). Letting the Fresnel coefficients $\mathcal{F}_{12}=\mathcal{F}_{23}=0$ go to zero for the homogeneous case, and $\mathcal{F}_{23}=0$ for a single plate in Eq. (B.1) yields

$$
\begin{aligned}
\operatorname{Im}\left[\mathbb{G}_{\text {plate }}-\mathbb{G}_{\text {bulk }}\right](\mathbf{r}, \mathbf{r}) & =\operatorname{Re} \frac{1}{2} \sum_{\sigma} \int \frac{\mathrm{d}^{2} \mathbf{q}_{\|}}{(2 \pi)^{2}} \frac{1}{p_{1}} e^{i \mathbf{q}_{1}^{+} \cdot \mathbf{r}} \hat{\sigma}_{1}^{+} \\
& \otimes\left[e^{-i \mathbf{q}_{1}^{+} \cdot \mathbf{r}} \hat{\sigma}_{1}^{+}+\mathcal{F}_{12}^{\sigma} e^{-i \mathbf{q}_{1}^{-} \cdot \mathbf{r}} \hat{\sigma}_{1}^{-}\right] \\
& -\operatorname{Re} \frac{i}{2} \sum_{\sigma} \int \frac{\mathrm{d}^{2} \mathbf{q}_{\|}}{(2 \pi)^{2}} \frac{1}{p_{1}} e^{i \mathbf{q}_{1}^{+} \cdot \mathbf{r}} \hat{\sigma}_{1}^{+} \\
& \otimes\left[e^{-i \mathbf{q}_{1}^{+} \cdot \mathbf{r}} \hat{\sigma}_{1}^{+}+e^{-i \mathbf{q}_{1}^{-} \cdot \mathbf{r}} \hat{\sigma}_{1}^{-}\right] \\
& =\operatorname{Re} \frac{1}{2} \int_{0}^{\infty} \mathrm{d} q_{\|} q_{\|} \frac{1}{(2 \pi)^{2}} \frac{1}{p_{1}} e^{2 i p_{1} z} \sum_{\sigma}\left(\mathcal{F}_{12}^{\sigma}-1\right) \int_{0}^{2 \pi} \mathrm{d} \varphi \hat{\sigma}_{1}^{+} \otimes \hat{\sigma}_{1}^{-},
\end{aligned}
$$

$\operatorname{Im}\left[\mathbb{G}_{\text {bulk }}-\mathbb{G}_{\text {plate }}\right](\mathbf{r}, \mathbf{r})=\operatorname{Re} \frac{1}{16 \pi} \int_{0}^{\infty} \mathrm{d} q_{\|} e^{2 i p_{1} z} \frac{q_{\|}}{p_{1}}$

$$
\times\left(\begin{array}{ccc}
\mathcal{F}_{12}^{s}-\mathcal{F}_{12}^{p} \frac{p_{1}^{2}}{k_{1}^{2}} & 0 & 0 \\
0 & \mathcal{F}_{12}^{s}-\mathcal{F}_{12}^{p} \frac{p_{1}^{2}}{k_{1}^{2}} & 0 \\
0 & 0 & 2\left(\mathcal{F}_{12}^{p}-1\right) \frac{q_{\|}^{2}}{k_{1}^{2}}
\end{array}\right) .
$$





\section{Casimir force and the Lifshitz formula}

\section{C.1. General Casimir force}

The electromagnetic stress tensor is given as

$$
\sigma_{i j}=\varepsilon_{0} E_{i} E_{j}+\frac{1}{\mu_{0}} B_{i} B_{j}-\frac{1}{2}\left(\varepsilon_{0} E^{2}+\frac{1}{\mu_{0}} B^{2}\right) \delta_{i j},
$$

and the force on an object is simply the surface integral around it,

$$
\mathbf{F}=\oint \sigma \cdot \mathrm{d} \mathbf{n}
$$

Taking a geometry shown in Figure C.1, the force on either of these plates due to the other can be written as

$$
\begin{aligned}
& P^{\mathrm{A} \rightarrow \mathrm{B}}=\left\langle\sigma_{z z}^{\mathrm{AB}}\right\rangle-\left\langle\sigma_{z z}^{\mathrm{B} 0}\right\rangle, \\
& P^{\mathrm{B} \rightarrow \mathrm{A}}=\left\langle\sigma_{z z}^{0 \mathrm{~A}}\right\rangle-\left\langle\sigma_{z z}^{\mathrm{AB}}\right\rangle .
\end{aligned}
$$

The Casimir pressure is then

$$
P^{\mathrm{AB}}=2\left\langle\sigma_{z z}^{\mathrm{AB}}\right\rangle-\left\langle\sigma_{z z}^{0 \mathrm{~A}}\right\rangle-\left\langle\sigma_{z z}^{\mathrm{B} 0}\right\rangle .
$$

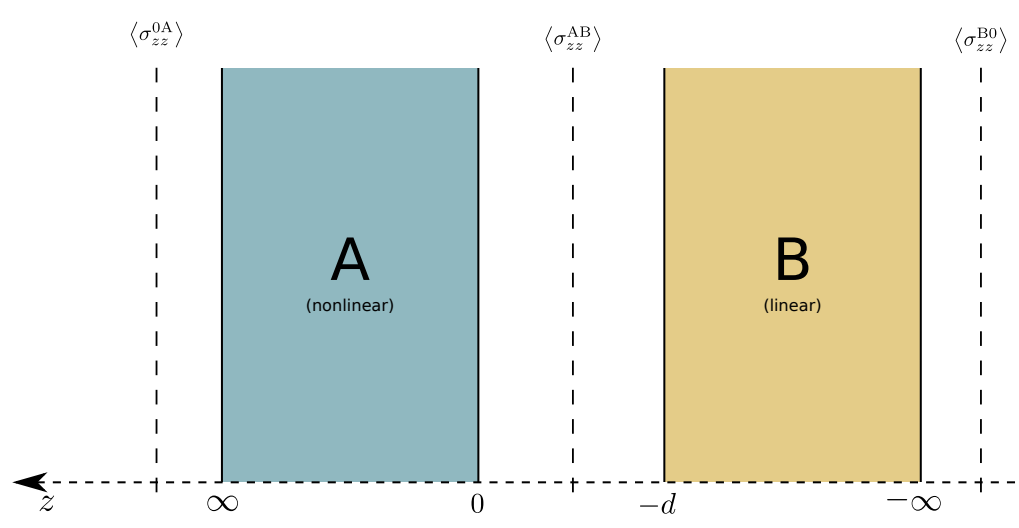

Figure C.1.: Parallel plate geometry. 
C. Casimir force and the Lifshitz formula

The $\left\langle\sigma_{z z}\right\rangle$ can be written as

$$
\left\langle\sigma_{z z}\right\rangle=\frac{\hbar}{2 \pi} \int_{0}^{\infty} \mathrm{d} \omega \frac{\omega^{2}}{c^{2}} \operatorname{coth}\left(\frac{\hbar \omega}{2 k_{\mathrm{B}} T}\right) \operatorname{Im} G_{\omega}
$$

where

$$
\begin{aligned}
G_{\omega} & =\mathcal{F}\left[\mathbb{G}_{\omega}+\frac{c^{2}}{\omega^{2}}\left[\nabla_{\mathbf{r}} \times \nabla_{\mathbf{r}^{\prime}} \times \mathbb{G}_{\omega}\right]\right]_{\mathbf{r}=\mathbf{r}^{\prime}=0+} \\
\mathcal{F}[\mathbb{X}] & =\mathbb{X}_{z z}-\mathbb{X}_{x x}-\mathbb{X}_{y y} .
\end{aligned}
$$

The full force is then

$$
\begin{aligned}
P^{\mathrm{AB}} & =2\left\langle\sigma_{z z}^{\mathrm{AB}}\right\rangle-\left\langle\sigma_{z z}^{0 \mathrm{~B}}\right\rangle-\left\langle\sigma_{z z}^{\mathrm{A} 0}\right\rangle \\
& =\frac{\hbar}{2 \pi} \int_{0}^{\infty} \mathrm{d} \omega \frac{\omega^{2}}{c^{2}} \operatorname{coth}\left(\frac{\hbar \omega}{2 k_{\mathrm{B}} T}\right) \Im G_{\omega}, \\
G_{\omega} & =\mathcal{F}\left[\mathbb{G}_{\omega}+\frac{c^{2}}{\omega^{2}}\left[\nabla_{\mathbf{r}} \times \nabla_{\mathbf{r}^{\prime}} \times \mathbb{G}_{\omega}\right]\right]_{\mathbf{r}=\mathbf{r}^{\prime}=0+}, \\
\mathbb{G} & =2 \mathbb{G}^{\mathrm{AB}}-\mathbb{G}^{0 \mathrm{~A}}-\mathbb{G}^{\mathrm{B} 0} .
\end{aligned}
$$

\section{C.2. Nonlinear Lifshitz formula}

(From the supplementary material of Ref. [85].)

Considering the experimental setup shown in Figure 3.7. For linear materials, the Casimir pressure $\left(P_{\text {lin }}\right)$ is given by the well known Lifshitz formula, and we provide here the additional term due to nonlinearities $\left(P_{\mathrm{nl}}\right)$. The pressure is a sum,

$$
P=P_{\text {lin }}+P_{\mathrm{nl}}
$$


where [85]

$$
\begin{aligned}
& P_{\text {lin }}=-\frac{1}{2 \pi} \varepsilon_{0} \operatorname{Re} \iint \mathrm{d} \omega \mathrm{d} q a(\omega) \frac{q p_{2}}{k_{2}^{2}} \sum_{\sigma \in\{\mathrm{s}, \mathrm{p}\}} \frac{\mathcal{F}_{21}^{\sigma} \mathcal{F}_{23}^{\sigma} e^{2 i p_{2} d}}{1-\mathcal{F}_{21}^{\sigma} \mathcal{F}_{23}^{\sigma} e^{2 i p_{2} d}}, \\
& P_{\mathrm{nl}}=\frac{3}{2^{8} \pi^{4}} \varepsilon_{0} \operatorname{Re} \iiint \int \mathrm{d} \omega \mathrm{d} \omega^{\prime} \mathrm{d} q \mathrm{~d} q^{\prime} \chi^{(3)}\left(-\omega, \omega, \omega^{\prime},-\omega^{\prime}\right) \\
& \times a(\omega) a\left(\omega^{\prime}\right)\left[S_{q, q^{\prime}}^{\omega, \omega^{\prime}}(d)+P_{q, q^{\prime}}^{\omega, \omega^{\prime}}(d)\right], \\
& S_{q, q^{\prime}}^{\omega, \omega^{\prime}}(d)=q q^{\prime} \frac{p_{2}^{2}}{p_{1}^{2}} \mathcal{F}_{23}^{s}\left(\frac{1-\mathcal{F}_{21}^{s}}{1-\mathcal{F}_{21}^{s} \mathcal{F}_{23}^{s} e^{2 i p_{2} d}}\right)^{2} \\
& \times\left[\frac{e^{2 i\left(p_{2}+p_{2}^{\prime}\right) d}}{\left(p_{1}+p_{1}^{\prime}\right) p_{1}^{\prime}} M_{x}\left(\omega^{\prime}, q^{\prime}, d\right)+\frac{e^{2 i\left(p_{2}-p_{2}^{\prime *}\right) d}}{\left(p_{1}-p_{1}^{\prime *}\right) p_{1}^{\prime *}} M_{x}^{*}\left(\omega^{\prime}, q^{\prime}, d\right)\right], \\
& P_{q, q^{\prime}}^{\omega, \omega^{\prime}}(d)=q q^{\prime} \frac{p_{2}^{2}}{p_{1}^{2}} \mathcal{F}_{23}^{p}\left(\frac{1-\mathcal{F}_{21}^{p}}{1-\mathcal{F}_{21}^{p} \mathcal{F}_{23}^{p} e^{2 i p_{2} d}}\right)^{2} \\
& \times\left[\frac{q^{2}}{k_{2}^{2}}\left[\frac{e^{2 i\left(p_{2}+p_{2}^{\prime}\right) d}}{\left(p_{1}+p_{1}^{\prime}\right) p_{1}^{\prime}} M_{z}\left(\omega^{\prime}, q^{\prime}, d\right)+\frac{e^{2 i\left(p_{2}-p_{2}^{\prime *}\right) d}}{\left(p_{1}-p_{1}^{\prime *}\right) p_{1}^{\prime *}} M_{z}^{*}\left(\omega^{\prime}, q^{\prime}, d\right)\right]\right. \\
& -\frac{p_{1}^{2}}{k_{2}^{2}}\left[\frac{e^{2 i\left(p_{2}+p_{2}^{\prime}\right) d}}{\left(p_{1}+p_{1}^{\prime}\right) p_{1}^{\prime}} M_{x}\left(\omega^{\prime}, q^{\prime}, d\right)+\frac{e^{2 i\left(p_{2}-p_{2}^{\prime *}\right) d}}{\left(p_{1}-p_{1}^{\prime *}\right) p_{1}^{\prime *}} M_{x}^{*}\left(\omega^{\prime}, q^{\prime}, d\right)(\mathrm{C}] \mathrm{1}, 7\right) \\
& M_{x}\left(\omega^{\prime}, q^{\prime}, d\right)=2\left(\frac{\mathcal{F}_{23}^{s \prime}-\mathcal{F}_{21}^{s \prime} \mathcal{F}_{21}^{s \prime} \mathcal{F}_{23}^{s \prime}}{1-\mathcal{F}_{21}^{s \prime} \mathcal{F}_{23}^{s \prime} e^{2 i p_{2}^{\prime} d}}\right)+\left(3 \frac{q^{\prime 2}}{k_{1}^{\prime 2}}-2\right)\left(\frac{\mathcal{F}_{23}^{p \prime}-\mathcal{F}_{21}^{p \prime} \mathcal{F}_{21}^{p \prime} \mathcal{F}_{23}^{p \prime}}{1-\mathcal{F}_{21}^{p \prime} \mathcal{F}_{23}^{p \prime} e^{2 i p_{2}^{\prime} d}}\right) \text {, } \\
& M_{z}\left(\omega^{\prime}, q^{\prime}, d\right)=\left(\frac{\mathcal{F}_{23}^{s \prime}-\mathcal{F}_{21}^{s \prime} \mathcal{F}_{21}^{s \prime} \mathcal{F}_{23}^{s \prime}}{1-\mathcal{F}_{21}^{s \prime} \mathcal{F}_{23}^{s \prime} e^{2 i p_{2}^{\prime} d}}\right)+\left(4 \frac{q^{\prime 2}}{k_{1}^{\prime 2}}-1\right)\left(\frac{\mathcal{F}_{23}^{p \prime}-\mathcal{F}_{21}^{p \prime} \mathcal{F}_{21}^{p \prime} \mathcal{F}_{23}^{p \prime}}{1-\mathcal{F}_{21}^{p \prime} \mathcal{F}_{23}^{p \prime} e^{2 i p_{2}^{\prime} d}}\right) \text {, }
\end{aligned}
$$

where the various quantities are given in Section B.1. The quantities $p_{n}^{\prime}, k_{n}^{\prime}, \mathcal{F}_{l n}^{s \prime}$, and $\mathcal{F}_{l n}^{p \prime}$ are defined in the same way, but using $\omega^{\prime}$ and $q^{\prime}$. The integration ranges are from zero to infinity with $a(\omega)=\frac{\hbar}{\pi \varepsilon_{0}} \frac{\omega^{2}}{c^{2}} \operatorname{coth}\left(\frac{\hbar \omega}{2 k_{\mathrm{B}} T}\right)$. 



\section{Heat transfer formula}

We give here the short derivation of the general heat transfer formula from the appendix of Ref. [86], which applies to linear, non-linear, and even non-reciprocal materials.

The total energy transmitted across a surface $\Sigma_{n}$ surrounding object $n$ is given by

$$
H_{n}=\oint_{\Sigma_{n}} d a\langle\mathbf{S}\rangle \cdot \mathbf{n}
$$

where $\langle\mathbf{S}\rangle=\langle\mathbf{E} \times \mathbf{H}\rangle$ is the time-average of the Poynting vector and $\mathbf{n}$ is a normal vector on $\Sigma_{n}$. The former can be expressed as,

$$
\langle\mathbf{S}\rangle=\int \frac{d \omega}{2 \pi} \operatorname{Re}\left\langle\mathbf{E} \times \mathbf{H}^{*}\right\rangle_{\omega} .
$$

Using the divergence theorem, Eq. (D.1) can be rewritten as

$$
H_{n}=\int \frac{d \omega}{2 \pi} \int_{V_{n}} d V \operatorname{Re}\left\langle\nabla \cdot\left(\mathbf{E}_{\omega} \times \mathbf{H}_{\omega}^{*}\right)\right\rangle
$$

For nonmagnetic materials $(\mu=1)$, it becomes

$$
\begin{aligned}
H_{n} & =\frac{1}{\mu_{0}} \int \frac{d \omega}{2 \pi} \frac{1}{\omega} \int_{V_{n}} d V \operatorname{Im}\left\langle\mathbf{E} \cdot(\nabla \times \nabla \times \mathbf{E})^{*}\right\rangle_{\omega} \\
& =-\frac{1}{\mu_{0}} \int \frac{d \omega}{2 \pi} \frac{1}{\omega} \operatorname{Tr}_{n} \operatorname{Im}\left[\mathbb{G}_{0}^{-1}\left\langle\mathbf{E} \otimes \mathbf{E}^{*}\right\rangle_{\omega}\right],
\end{aligned}
$$

where $\operatorname{Tr}_{n}$ denotes a trace, which is restricted to volume $V_{n}$. It can be shown that, even without micro-reversibility, there is no heat transfer if the temperatures are equal.

Using Eq. (4.17) for the correlator with unequal temperatures and subtracting the contribution of a pseudo-system, where all bodies are at a temperature $T_{n}$ (therefore giving no contribution to heat transfer), the final general form of the heat radiation equation 
D. Heat transfer formula

can be obtained,

$$
\begin{aligned}
H_{n} & =-\frac{1}{4 \mu_{0}} \sum_{m=0}^{N} \int \frac{d \omega}{2 \pi} \frac{1}{\omega}\left[b_{n}(\omega)-b_{m}(\omega)\right] \\
& \times \operatorname{Tr}\left[\left(\tilde{\mathbb{V}}_{n}\right)_{\mathrm{AH}} \tilde{\mathbb{G}}\left(\tilde{\mathbb{V}}_{m}\right)_{\mathrm{AH}} \tilde{\mathbb{G}}^{\dagger}\right] .
\end{aligned}
$$

Note that the terms with $T_{m}=T_{n}$ (including $m=n$ ) explicitly do not contribute to heat radiation. Furthermore, if $\tilde{\mathbb{V}}$ and therefore $\widetilde{\mathbb{G}}$ are symmetric (implying microreversibility [29]), then Eq. (D.5) can be further simplified,

$$
\begin{aligned}
H_{n}= & \frac{1}{\mu_{0}} \sum_{m=0}^{N} \int \frac{d \omega}{2 \pi} \frac{1}{\omega}\left[b_{m}(\omega)-b_{n}(\omega)\right] \\
& \times \operatorname{Tr}\left(\operatorname{Im}\left[\tilde{\mathbb{V}}_{n}\right] \tilde{\mathbb{G}} \operatorname{Im}\left[\tilde{\mathbb{V}}_{m}\right] \tilde{\mathbb{G}}^{*}\right) .
\end{aligned}
$$




\section{Bibliography}

[1] A. A. Abrikosov, L. P. Gorkov, I. E. Dzyaloshinski, R. A. Silverman, and G. H. Weiss, Methods of Quantum Field Theory in Statistical Physics, Phys. Today 17 (1964), no. 4, 78-80.

[2] D. Ahn and S.-1. Chuang, Calculation of linear and nonlinear intersubband optical absorptions in a quantum well model with an applied electric field, IEEE J. Quantum Electron. 23 (1987), no. 12, 2196-2204.

[3] A. Altland and B. D. Simons, Condensed matter field theory, Cambridge University Press, 2010.

[4] D. Andrews, R. Crisp, and D. Bradshaw, Optically induced inter-particle forces: From the bonding of dimers to optical electrostriction in molecular solids, J. Phys. B At. Mol. Opt. Phys. 39 (2006), no. 15, S637-S650.

[5] M. Antezza, L. P. Pitaevskii, S. Stringari, and V. B. Svetovoy, Casimir-Lifshitz force out of thermal equilibrium, Phys. Rev. A 77 (2008), no. 2, 022901.

[6] R. M. Balachandran, N. M. Lawandy, and J. A. Moon, Theory of laser action in scattering gain media, Opt. Lett. 22 (2008), no. 5, 319.

[7] R. Bavli and Y. B. Band, Nonlinear absorption and dispersion in a two-level system with permanent dipole moments, Phys. Rev. A 43 (1991), no. 9, 5039-5043.

[8] P. Ben-Abdallah and K. Joulain, Fundamental limits for noncontact transfers between two bodies, Phys. Rev. B - Condens. Matter Mater. Phys. 82 (2010), no. 12, 1-4.

[9] S. A. Biehs, E. Rousseau, and J. J. Greffet, Mesoscopic description of radiative heat transfer at the nanoscale, Phys. Rev. Lett. 105 (2010), no. 23, 3-6.

[10] A. Boardman, V. Grimalsky, Y. Kivshar, S. Koshevaya, M. Lapine, N. Litchinitser, V. Malnev, M. Noginov, Y. Rapoport, and V. Shalaev, Active and tunable metamaterials, Laser Photon. Rev. 5 (2011), no. 2, 287-307.

[11] C. F. Bohren and D. R. Huffman, Absorption and scattering of light by small particles, John Wiley \& Sons, 2008.

[12] M. Bordag, G. L. Klimchitskaya, U. Mohideen, and V. M. Mostepanenko, Advances in the Casimir Effect, vol. 145, Oxford University Press, Oxford, may 2009. 
Bibliography

[13] R. W. Boyd, Nonlinear Optics, Elsevier, 2008.

[14] T. H. Boyer, Temperature dependence of Van der Waals forces in classical electrodynamics with classical electromagnetic zero-point radiation, Phys. Rev. A 11 (1975), no. 5, 1650-1663.

[15] G. Bressi, G. Carugno, R. Onofrio, and G. Ruoso, Measurement of the Casimir Force between Parallel Metallic Surfaces, Phys. Rev. Lett. 88 (2002), no. 4, 041804.

[16] R. Budwig, Refractive index matching methods for liquid flow investigations, Exp. Fluids 17 (1994), no. 5, 350-355.

[17] C. Cao, M. van Caspel, and A. R. Zhitnitsky, Topological Casimir effect in Maxwell electrodynamics on a compact manifold, Phys. Rev. D 87 (2013), no. 10, 105012.

[18] G. M. Carter, M. K. Thakur, Y. J. Chen, and J. V. Hryniewicz, Time and wavelength resolved nonlinear optical spectroscopy of a polydiacetylene in the solid state using picosecond dye laser pulses, Appl. Phys. Lett. 47 (1985), no. 5, 457-459.

[19] H. Casimir, On the attraction between two perfectly conducting plates, Proc. K. Ned. Akad. 51 (1948), no. April, 793-795.

[20] H. B. G. Casimir and D. Polder, The Influence of Retardation on the London-van der Waals Forces, Phys. Rev. 73 (1948), no. 4, 360-372.

[21] S. J. Chang, Quantum fluctuations in a 4 field theory. I. Stability of the vacuum, Phys. Rev. D 12 (1975), no. 4, 1071-1088.

[22] F. Chen, G. L. Klimchitskaya, V. M. Mostepanenko, and U. Mohideen, Control of the Casimir force by the modification of dielectric properties with light, Phys. Rev. B 76 (2007), no. 3, 035338.

[23] M. V. Cougo-Pinto, C. Farina, M. R. Negrão, and A. C. Tort, Bosonic Casimir effect in external magnetic field, J. Phys. A. Math. Gen. 32 (1999), no. 24, 4457-4462.

[24] D. S. Dean, V. Démery, V. A. Parsegian, and R. Podgornik, Out-of-equilibrium relaxation of the thermal Casimir effect in a model polarizable material, Phys. Rev. E 85 (2012), no. 3, 031108.

[25] D. S. Dean, B.-S. Lu, A. C. Maggs, and R. Podgornik, Nonequilibrium Tuning of the Thermal Casimir Effect, Phys. Rev. Lett. 116 (2016), no. 24, 240602.

[26] D. S. Dean, V. A. Parsegian, and R. Podgornik, Fluctuation of thermal van der Waals forces due to dipole fluctuations, Phys. Rev. A 87 (2013), no. 3, 032111.

[27] P. D. Drummond, Electromagnetic quantization in dispersive inhomogeneous nonlinear dielectrics, Phys. Rev. A 42 (1990), no. 11, 6845-6857.

[28] I. E. Dzyaloshinskii, E. M. Lifshitz, and L. P. Pitaevskii, General Theory of Van Der Waals'Forces, Physics-Uspekhi 4 (1961), no. 2, 153-176. 
[29] W. Eckhardt, Macroscopic theory of electromagnetic fluctuations and stationary radiative heat transfer, Phys. Rev. A 29 (1984), no. 4, 1991-2003.

[30] J. F. Federici, Review of Four- Wave Mixing and Phase Conjugation in Plasmas, IEEE Trans. Plasma Sci. 19 (1991), no. 4, 549-564.

[31] J. F. Federici and D. K. Mansfield, Degenerate four-wave mixing and phase conjugation in a collisional plasma, J. Opt. Soc. Am. B 3 (1986), no. 11, 1588.

[32] C. D. Fosco and L. E. Oxman, Vacuum fluctuations in the presence of nonlinear boundary conditions, Phys. Rev. D 92 (2015), no. 12, 125021.

[33] K. Fukumi, A. Chayahara, K. Kadono, T. Sakaguchi, Y. Horino, M. Miya, K. Fujii, J. Hayakawa, and M. Satou, Gold nanoparticles ion implanted in glass with enhanced nonlinear optical properties, J. Appl. Phys. 75 (1994), no. 6, 3075-3080.

[34] M. Gad-el Hak, The MEMS Handbook, Mechanical Engineering Series, CRC Press, 2001.

[35] A. Gambassi, A. Maciołek, C. Hertlein, U. Nellen, L. Helden, C. Bechinger, and S. Dietrich, Critical Casimir effect in classical binary liquid mixtures, Phys. Rev. E 80 (2009), no. 6, 061143.

[36] Y. Hamanaka, A. Nakamura, N. Hayashi, and S. Omi, Dispersion curves of complex third-order optical susceptibilities around the surface plasmon resonance in $\mathrm{Ag}$ nanocrystal-glass composites, J. Opt. Soc. Am. B 20 (2003), no. 6, 1227-1232.

[37] M. Hillery and L. Mlodinow, Quantized fields in a nonlinear dielectric medium: A microscopic approach, Phys. Rev. A 55 (1997), no. 1, 678-689.

[38] J. D. Jackson, Classical Electrodynamics, 3rd Edition, Wiley, New York, 1998.

[39] P. Johansson, Electromagnetic Green's function for layered systems: Applications to nanohole interactions in thin metal films, Phys. Rev. B 83 (2011), no. 19, 195408.

[40] M. Kardar, Statistical physics of fields, Cambridge University Press, 2007.

[41] L. Karvonen, J. Rönn, S. Kujala, Y. Chen, A. Säynätjoki, A. Tervonen, Y. Svirko, and S. Honkanen, High non-resonant third-order optical nonlinearity of Ag-glass nanocomposite fabricated by two-step ion exchange, Opt. Mater. (Amst). 36 (2013), no. $2,328-332$.

[42] M. Kauranen and A. V. Zayats, Nonlinear plasmonics, Nat. Photonics 6 (2012), no. 11, 737-748.

[43] C. Khandekar, A. Pick, S. G. Johnson, and A. W. Rodriguez, Radiative heat transfer in nonlinear Kerr media, Phys. Rev. B 91 (2015), no. 11, 115406.

[44] C. Khandekar and A. W. Rodriguez, Near-field thermal upconversion and energy transfer through a Kerr medium, Opt. Express 25 (2017), no. 19, 23164. 
[45] F. Kheirandish, E. Amooghorban, and M. Soltani, Finite-temperature Casimir effect in the presence of nonlinear dielectrics, Phys. Rev. A 83 (2011), no. 3, 032507.

[46] K. Kim, B. Song, V. Fernández-Hurtado, W. Lee, W. Jeong, L. Cui, D. Thompson, J. Feist, M. T. H. Reid, F. J. García-Vidal, J. C. Cuevas, E. Meyhofer, and P. Reddy, Radiative heat transfer in the extreme near field, Nature 528 (2015), no. 7582, 387-391.

[47] A. Kittel, W. Müller-Hirsch, J. Parisi, S.-A. Biehs, D. Reddig, and M. Holthaus, Near-Field Heat Transfer in a Scanning Thermal Microscope, Phys. Rev. Lett. 95 (2005), no. 22, 224301.

[48] Y. L. Klimontovich, Alternative description of stochastic processes in nonlinear systems. "Kinetic form" of master and Fokker-Planck equations, Phys. A Stat. Mech. its Appl. 182 (1992), no. 1-2, 121-132.

[49] Y. L. Klimontovich, Nonlinear Brownian motion, Physics-Uspekhi 37 (1994), no. 8, 737-766.

[50] M. Krech, The Casimir effect in critical systems, World Scientific, 1994.

[51] M. Krüger, G. Bimonte, T. Emig, and M. Kardar, Trace formulas for nonequilibrium Casimir interactions, heat radiation, and heat transfer for arbitrary objects, Phys. Rev. B 86 (2012), no. 11, 115423.

[52] M. Krüger, T. Emig, and M. Kardar, Nonequilibrium Electromagnetic Fluctuations: Heat Transfer and Interactions, Phys. Rev. Lett. 106 (2011), no. 21, 210404.

[53] R. Kubo, The fluctuation-dissipation theorem, Reports Prog. Phys. 29 (1966), no. 1, 306.

[54] S. M. Kuebler, R. G. Denning, and H. L. Anderson, Large Third-Order Electronic Polarizability of a Conjugated Porphyrin Polymer, J. Am. Chem. Soc. 122 (2000), no. 2, 339-347.

[55] D. Kysylychyn, V. Piatnytsia, and V. Lozovski, Electrodynamic interaction between a nanoparticle and a surface of a solid, Phys. Rev. E 88 (2013), no. 5, 052403.

[56] S. K. Lamoreaux, Demonstration of the Casimir Force in the 0.6 to $6 \mu m$ Range, Phys. Rev. Lett. 78 (1997), no. 1, 5-8.

[57] M. Lapine, I. V. Shadrivov, and Y. S. Kivshar, Colloquium: Nonlinear metamaterials, Rev. Mod. Phys. 86 (2014), no. 3, 1093-1123.

[58] M. L. Levin and S. M. Rytov, Theory of equilibrium thermal fluctuations in electrodynamics, 1967.

[59] E. M. Lifshitz, The theory of molecular attractive forces between solids, Sov. Phys. JETP 2 (1956), 73-83. 
[60] B. A. Lippmann and J. Schwinger, Variational Principles for Scattering Processes. I, Phys. Rev. 79 (1950), no. 3, 469-480.

[61] K. Makhnovets and A. Kolezhuk, On Short-Range Enhancement of Van-der-Waals Forces, Materwiss. Werksttech. 47 (2016), no. 2-3, 222-228.

[62] R. Matloob, Electromagnetic field quantization in an absorbing medium, Phys. Rev. A 60 (1999), no. 1, 50-55.

[63] P. W. Milonni, The Quantum Vacuum: An Introduction to Quantum Electrodynamics, 2013.

[64] U. Mohideen and A. Roy, Precision Measurement of the Casimir Force from 0.1 to $0.9 \mu m$, Phys. Rev. Lett. 81 (1998), no. 21, 4549-4552.

[65] B. Müller, R. Incardone, M. Antezza, T. Emig, and M. Krüger, Many-body heat radiation and heat transfer in the presence of a nonabsorbing background medium, Phys. Rev. B 95 (2017), no. 8, 1-18.

[66] B. Müller and M. Krüger, Anisotropic particles near surfaces: Propulsion force and friction, Phys. Rev. A 93 (2016), no. 3, 1-12.

[67] L. Novotny and B. Hecht, Principles of nano-optics, 2009.

[68] J. M. Obrecht, R. J. Wild, M. Antezza, L. P. Pitaevskii, S. Stringari, and E. A. Cornell, Measurement of the Temperature Dependence of the Casimir-Polder Force, Phys. Rev. Lett. 98 (2007), no. 6, 063201.

[69] R. S. Ottens, V. Quetschke, S. Wise, A. A. Alemi, R. Lundock, G. Mueller, D. H. Reitze, D. B. Tanner, and B. F. Whiting, Near-Field Radiative Heat Transfer between Macroscopic Planar Surfaces, Phys. Rev. Lett. 107 (2011), no. 1, 014301.

[70] J. B. Pendry, Shearing the vacuum - Quantum friction, J. Phys. Condens. Matter 9 (1997), no. 47, 10301-10320.

[71] D. Polder and M. Van Hove, Theory of Radiative Heat Transfer between Closely Spaced Bodies, Phys. Rev. B 4 (1971), no. 10, 3303-3314.

[72] S. J. Rahi, T. Emig, N. Graham, R. L. Jaffe, and M. Kardar, Scattering theory approach to electrodynamic Casimir forces, Phys. Rev. D 80 (2009), no. 8, 085021.

[73] D. Robaschik, K. Scharnhorst, and E. Wieczorek, Radiative corrections to the Casimir pressure under the influence of temperature and external fields, Ann. Phys. (N. Y). 174 (1987), no. 2, 401-429.

[74] A. W. Rodriguez, M. T. H. Reid, and S. G. Johnson, Fluctuating-surface-current formulation of radiative heat transfer for arbitrary geometries, Phys. Rev. B - Condens. Matter Mater. Phys. 86 (2012), no. 22, 1-5. 
Bibliography

[75] E. Rosencher and P. Bois, Model system for optical nonlinearities: Asymmetric quantum wells, Phys. Rev. B 44 (1991), no. 20, 11315-11327.

[76] E. Rousseau, A. Siria, G. Jourdan, S. Volz, F. Comin, J. Chevrier, and J.-J. Greffet, Radiative heat transfer at the nanoscale, Nat. Photonics 3 (2009), no. 9, 514-517.

[77] S. M. Rytov, Y. A. Kravtsov, and V. I. Tatarski, Principles of statistical radiophysics 3, 1989.

[78] A. Salam, On the effect of a radiation field in modifying the intermolecular interaction between two chiral molecules., J. Chem. Phys. 124 (2006), no. 1, 14302.

[79] S. Scheel and D.-G. Welsch, Quantum Theory of Light and Noise Polarization in Nonlinear Optics, Phys. Rev. Lett. 96 (2006), no. 7, 073601.

[80] M. Schubert and B. Wilhelmi, Nonlinear optics and quantum electronics, WileyInterscience, New York, 1986.

[81] S. Shen, A. Narayanaswamy, and G. Chen, Surface Phonon Polaritons Mediated Energy Transfer between Nanoscale Gaps, Nano Lett. 9 (2009), no. 8, 2909-2913.

[82] Y. Shi, Z. Yu, and S. Fan, Limitations of nonlinear optical isolators due to dynamic reciprocity, Nat. Photonics 9 (2015), no. 6, 388-392.

[83] H. Soo, Fluctuational Electrodynamics for Nonlinear Media, Master thesis, Universität Stuttgart, 2015.

[84] H. Soo, D. S. Dean, and M. Krüger, Particles with nonlinear electric response: Suppressing van der Waals forces by an external field, Phys. Rev. E 95 (2017), no. 1, 012151.

[85] H. Soo and M. Krüger, Fluctuational electrodynamics for nonlinear media, EPL 115 (2016), no. 4, 41002.

[86] H. Soo and M. Krüger, Fluctuational electrodynamics for nonlinear materials in and out of thermal equilibrium, Phys. Rev. B 97 (2018), no. 4, 045412.

[87] W. H. Southwell, Pyramid-array surface-relief structures producing antireflection index matching on optical surfaces, J. Opt. Soc. Am. A 8 (1991), no. 3, 549.

[88] C. I. Sukenik, M. G. Boshier, D. Cho, V. Sandoghdar, and E. A. Hinds, Measurement of the Casimir-Polder force, Phys. Rev. Lett. 70 (1993), no. 5, 560-563.

[89] I. Szalai, S. Nagy, and S. Dietrich, Nonlinear dielectric effect of dipolar fluids, J. Chem. Phys. 131 (2009), no. 15, 154905.

[90] G. Torricelli, P. J. van Zwol, O. Shpak, C. Binns, G. Palasantzas, B. J. Kooi, V. B. Svetovoy, and M. Wuttig, Switching Casimir forces with phase-change materials, Phys. Rev. A 82 (2010), no. 1, 010101. 
[91] L. Tsang, D. Ahn, and S. L. Chuang, Electric field control of optical second-harmonic generation in a quantum well, Appl. Phys. Lett. 52 (1988), no. 9, 697-699.

[92] W.-K. Tse and A. H. MacDonald, Quantized Casimir Force, Phys. Rev. Lett. 109 (2012), no. 23, 236806.

[93] V. N. Tsytovich, Nonlinear effects in a plasma, Sov. Phys. Uspekhi 9 (1967), no. 6, 805-836.

[94] L. D. Tzuang, K. Fang, P. Nussenzveig, S. Fan, and M. Lipson, Non-reciprocal phase shift induced by an effective magnetic flux for light, Nat. Photonics 8 (2014), no. 9, 701-705.

[95] N. Van Kampen, Fluctuation phenomena in solids, New York-London RE Burgess (1965).

[96] N. G. van Kampen, Thermal Fluctuations in a Nonlinear System, Phys. Rev. 110 (1958), no. 2, 319-323.

[97] J. Wang, X. Zhang, S.-Y. Pei, and D.-H. Liu, Tunable Casimir forces by means of the external magnetic field, Phys. Rev. A 73 (2006), no. 4, 042103.

[98] S. Wiederseiner, N. Andreini, G. Epely-Chauvin, and C. Ancey, Refractive-index and density matching in concentrated particle suspensions: a review, Exp. Fluids $\mathbf{5 0}$ (2011), no. 5, 1183-1206.

[99] H. Yildirim and M. Tomak, Nonlinear optical properties of a Pöschl-Teller quantum well, Phys. Rev. B 72 (2005), no. 11, 115340.

[100] L. Zhang, Y.-M. Chi, and J.-J. Shi, Second-order nonlinear optical susceptibilities induced by built-in electric field in wurtzite nitride double quantum wells, Phys. Lett. A 366 (2007), no. 3, 256-261. 



\section{Acknowledgments}

The journey that culminated in this thesis has been extremely rewarding, but also at times difficult and confusing. I could not have done it without the help of many amazing people on my way.

First and foremost, I am deeply grateful to my supervisor Prof. Matthias Krüger, with whom we embarked on this road of fluctuations in nonlinear systems almost five years ago. His focus and drive has been a constant inspiration, which, together with the unwavering support and patience, has helped me develop from a physics student into a young scientist.

For the discussions about physics and life, I would like to thank my fellow group members, particularly Boris, Chris, and Kiryl. Also for attending my talks and seminars and giving useful feedback.

I want to thank Prof. M. Kardar for hosting me in MIT, Boston. I am most grateful to him and also Professors S. Dietrich, D. Dean, and T. Emig for their invaluable insights and help at various stages of this project.

I thank my family and friends for their support and understanding. I am especially grateful to Divya, who unfairly had to absorb most of my frustrations and doubt. She always had my back and inspired me to do my best.

For reading and giving feedback on the manuscript to get it over the final line, I thank Divya, Chris, and Taavi. 



\section{Curriculum vitae}

$\begin{array}{ll}\text { Name } & \text { Heino Soo } \\ \text { Date of birth } & \text { July 12, 1990 } \\ \text { Place of birth } & \text { Jõgeva, Estonia } \\ \text { Nationality } & \text { Estonian }\end{array}$

\section{Education}

Jan 2018 - Feb 2019

Oct 2015 - Jan 2018

Oct 2013 - Sep 2015

Sep 2009 - Jun 2013

B.Sc. in Physics
Doctoral studies (cont.)

University of Göttingen, Germany

Doctoral studies

University of Stuttgart, Germany

M.Sc. in Physics

University of Stuttgart, Germany

Thesis: "Fluctuational electrodynamics in nonlinear media"

University of Tartu, Estonia

Thesis: "Phonon mediated electron-electron interaction in twoband superconductors"

\section{Conferences}

Aug 2018

May 2018
Progress in Electromagnetics Research Symposium, Toyama, Japan Talk: "Fluctuational electrodynamics for nonlinear materials" WE-Heraeus-Seminar on Fluctuation-Induced Phenomena in Complex Systems, Bad Honnef, Germany

Poster: "Fluctuational electrodynamics for nonlinear materials" 
Progress in Electromagnetics Research Symposium, St. Petersburg, Russia

Talk: "Fluctuational electrodynamics for nonlinear materials"

Apr 2016

WE-Heraeus-Seminar on Heat Transfer and Heat Conduction on the Nanoscale, Bad Honnef, Germany

Poster: "Fluctuational electrodynamics for nonlinear materials"

\section{Publications}

Jan 2018

Sep 2016

Jan 2017
Heino Soo and Matthias Krüger, Fluctuational electrodynamics for nonlinear materials in and out of thermal equilibrium, Phys. Rev. B 97, 4 (2018), pp. 045412.

Heino Soo and Matthias Krüger, Fluctuational electrodynamics for nonlinear media, EPL 115, 4 (2016), pp. 41002.

Heino Soo, David S. Dean, and Matthias Krüger, Particles with nonlinear electric response: Suppressing van der Waals forces by an external field, Phys. Rev. E 95, 1 (2017), pp. 012151.

\section{Scholarships}

Oct 2013 - Sep 2015

Oct 2012 - Jun 2013
DAAD Study Scholarship for Graduates

Rotalia Foundation Scholarship 